\title{
As a Newly-Discovered Component in Tumor
}

Microenvironment RUFY4 Participates in Regulation

of T cell via Cytokine-Interaction and Predicts

Prognosis and Immunotherapy Responsiveness of Clear Cell Renal Carcinoma Patients

\section{Daojia Miao}

Huazhong University of Science and Technology Tongji Medical College: Wuhan Union Hospital

Jian Shi

Huazhong University of Science and Technology Tongji Medical College First Clinical College: Wuhan Union Hospital

\section{Zhiyong Xiong}

Huazhong University of Science and Technology Tongji Medical College First Clinical College: Wuhan Union Hospital

\section{Changfei Yuan}

Huazhong University of Science and Technology Tongji Medical College First Clinical College: Wuhan Union Hospital

\section{Wen Xiao}

Huazhong University of Science and Technology Tongji Medical College First Clinical College: Wuhan Union Hospital

\section{Xiangui Meng}

Huazhong University of Science and Technology Tongji Medical College First Clinical College: Wuhan Union Hospital

\section{Zhixian Chen}

Huazhong University of Science and Technology Tongji Medical College First Clinical College: Wuhan Union Hospital

\section{Qingyang Lv}

Huazhong University of Science and Technology Tongji Medical College First Clinical College: Wuhan Union Hospital

\section{Kairu Xie}

Huazhong University of Science and Technology Tongji Medical College First Clinical College: Wuhan Union Hospital

\section{Hongmei Yang}

Huazhong University of Science and Technology Tongji Medical College First Clinical College 
Xiao-ping Zhang ( $\nabla$ xzhang@hust.edu.cn )

Huazhong University of Science and Technology

\section{Research}

Keywords: Clear cell renal cell carcinoma (ccRCC), Immunotherapy, T cells regulation, PD-1 blockade, Prognostic signatures

Posted Date: December 11th, 2020

DOl: https://doi.org/10.21203/rs.3.rs-125317/v1

License: (c) (i) This work is licensed under a Creative Commons Attribution 4.0 International License. Read Full License 


\section{Abstract}

Background: clear cell renal cell carcinoma (ccRCC) is one of the most lethal kinds of malignancies in urinary system and the existing immunotherapy have not achieved satisfactory outcomes. Therefore, this study aims to establish a brand-new gene signature for immune-infiltration and clinical outcome (overall survival and immunotherapy responsiveness) of patients with ccRCC.

Methods: Based on RNA sequencing data and clinical information in the Cancer Genome Atlas Project (TCGA) database, we investigated proportions of immune cells in 611 samples by an online tool CIBERSORTX. Multivariate survival analysis was used to determine crucial survival-associated immune cells and immune-infiltration-related genes (IIRGs). Next ROC analysis was carried on to evaluate the ability of IIRGs to distinguish patients and functional enrichment analysis were implemented to explore potential interaction network between immune cells and IIRGs.

Results: T cells follicular helper (TFHs) and T cells regulatory (Tregs) were highly infiltrated in the tumor microenvironment and their abundance ratios were independent prognostic factors for overall survival. Among IIRGs of TFHs and TREGs, RUFY4 was found to be highly activated in tumor microenvironment and its co-expression network was enriched in regulation of T cells via cytokine-cytokine receptor interactions.

Conclusion: These two cells and RUFY4, considered prognostic biomarkers and immunotherapeutic predictors of ccRCC patients, might also simultaneously affect the regulatory network in tumor microenvironment (TME) through cytokine interactions.

\section{Background}

Kidney Cancer, whose share in global cancer cases is approximately $2.2 \%$ when 1.8 million new diagnoses are estimated across 20 world folks, causes about 175,100 deaths in 2018[1]. Among all kidney cancer subtypes, clear cell renal cell carcinoma (ccRCC) is the predominant one, representing more than $70 \%$ of all RCC cases[2]. In the past decade, antiangiogenic drugs targeting VEGF, mTOR inhibitors and immune checkpoint inhibitors $(\mathrm{ICl})$ have quickly expanded the treatment options for metastatic ccRCC[3]. Though great improvement in managing ccRCC has been made via specific cellular and molecular targets, the prognosis of patients is still unsatisfactory.

The interaction between cancer cells and immune cells in tumor microenvironment (TME) has been a new hallmark of cancer since 2011[4]. As an indispensable part of TME, immune-infiltration cells have been shown to play a crucial role in the occurrence and development of tumors[5]. In previous studies, ccRCC has been also considered as a highly immune-infiltration tumor and different immune-infiltration was correlated with distinct clinicopathologic features [6, 7]. Studies by Ueda, K[8]. and Mikami, S. [9] have suggested that immune-infiltrated-related genes (IIRGs) are associated not only with the response to immunotherapy, but also with the clinical outcome of ccRCC patients. However, their practical effect and specificity in predicting prognosis and therapeutic potential remain problematic. Therefore, in order to 
improve the survival time and life quality of ccRCC patients and to provide worthy information to conduct the precise individual treatment, novel immune predictors and prognostic indicators are urgently needed.

RUN and FYVE domain-containing proteins (RUFYs), consisting of an N-terminal RUN domain and a Phosphatidylinositol 3-phosphate (PI3P)-interacting C-terminal FYVE domain, have taken center stage in the oncology field[10]. RUFY1 is proved to interact with podocalyxin-like (PODXL) protein, an iron exchanger regulatory factor in the membrane protein complex associated with poor prognosis of different cancers $[11,12]$. As for RUFY2, Shin N.[13] suggests that one of the extremely frequently mutated genes in colorectal cancer and research by Zheng Z.[14] and Staubitz [15] shows RUFY2 have participated in tumorigenesis in lung adenocarcinoma and papillary thyroid carcinoma. RUFY3 is the last well-known protein in the family and its dysregulation is connected to growth, invasion and metastasis of lung adenocarcinoma[16] and colorectal cancer[17]. Although RUFY4 is reported to be the first molecule that influences autophagy and endosome dynamics in a subset of immune cells and RUFY4 is also an important factor that defines the nature of the response of cells to the direct immune environment [18], there is no strong evidence that RUFY4 is related to any type of cancer.

Based on The Cancer Genome Atlas Project (TCGA) database, this study quantified the composition of immune cell in ccRCC and combined their clinicopathologic attributes to explore the relationships with overall survival (OS) rates. Furthermore, we identified IIRGs that could simultaneously reflect the degree of immune-infiltration and predict patient prognosis and immunotherapy responsiveness. Also, the present study offers a novel method for researchers to creatively tap into immunotherapeutic cells and gene signatures for all other cancers.

\section{Materials And Methods}

\section{Data source and cleaning}

The ccRCC RNA-Seq data (FPKM and COUNT formats) in this study were obtained from TCGA database via the Data transfer tool on the website. Samples information and corresponding clinical data which consist of gender, age, clinical TNM stage, histopathological grade, survival time, etc. were directly collected form the website. Then, two gene expression matrices of COUNT and FPKM and one clinical information table were constructed through $R$ software (version: $R \times 64$ 4.0.3) which a free software environment for statistical computing and graphics. All subsequent analyses and results were based on those three datasets, whose analytical procedures was shown in Fig. 1.

\section{Establishment immune-infiltration landscape}

CIBERSORTx is a computational online tool to accurately infer cell type abundance from RNA profiles of intact tissues[19]. Compared with CIBERSORT, CIBERSORTx not only tell researchers cell types, but also cell status affected by tumor. After uploading the RNA-Seq (FPKM gene expression matrix) of ccRCC, this study conduct CIBERSORTx analysis online on the website (https://cibersortx.stanford.edu/) according to the following parameters: 
sigmatrix: LM22.update-gene-symbols.txt, perm: 500, verbose: TRUE, rmbatchBmode: TRUE, QN: FALSE

In sum, 609 samples were selected with $\mathrm{P} \leq 0.05$ and each sample was equipped with the relative abundance matrix of 22 common immune cell types. According to the low quartile of Root Mean Squared Error in each group, a quarter of the sample were selected and displayed by $\mathrm{R}$ software. And then $\mathrm{R}$ packages "pheatmap", "barplot" and "vioplot" were installed in R software to establish the immuneinfiltration landscape.

\section{Identification survival-associated immune cells}

On basis of the fractions of 22 immune cells, K-M analysis of OS rate was performed for those immune cells types whose immune-infiltration had apparent difference between tumor and its adjacent normal tissues. Among Kaplan-Meier survival analysis, the cut-off was set as the upper and lower quartiles while statistically significant means that $P$ values less than 0.05 .

\section{Relationship between clinical attributes and survival- associated immune cells}

To identify immune cells with clinical-guide valve, the relationship between the fractions of immune cells and clinical stage, histopathological grade, TMN stage were analyzed R package "readr" on R software. Statistically significant differences between two groups and among more than two groups were determined using Wilcox test and Kruskal test, respectively. By putting the fractions of immune cells and the clinical attributes together, this study could better figure out the prognostic valve of survivalassociated immune cells.

\section{Screening crucial survival-associated immune cells in TME}

The relationship between immune cells were carried out based on the proportions of each cells. Spearman correlation analysis was chosen with the cut-off of P-valve setting as 0.05 and $R$ packages "corrplot" was used to plot the correlation indices with various colors. Univariate survival analysis was used to verify the prognostic predictive effect of known factors, such as age, gender, stage $T$, stage $M$, etc. This study created a multivariate model which were adjusted for those factors whose $H R>1$. By setting the cut-off as the median of abundance ratios, 537 samples were distributed into high and low groups. Then the abundance of each immune cells was added as a new binary variable to multivariate model for cox survival analysis.

\section{Identification and Enrichment Analysis of IIRGs}

In previous step, two crucial survival-associated immune cells were identified. The RNA-Seq Count data were used to figure out the differentially expression genes, which were named IIRGs in this study, between the high and low immune-infiltration groups. Also, differentially expression genes between tumor and adjacent normal tissues were identified. The whole procedure was conducted on $\mathrm{R}$ software by $\mathrm{R}$ packages "DEseq2" with the following condition: 
cut-off $>$ mean $(|\log F C|)+\mathrm{sd}(|\log F C|), P<0.01$

R packages "EnhancedVolcano" was used to visualize these differentially expression genes. Next this study carried out gene functional classification and gene annotation of Immune-associated genes on the website DAVID by the methods of GO and KEGG analysis. P-value is the mirror reflecting the significance of enrichment results with a cut-off setting as 0.05 . R packages "ggplot2" were used to visualize the top 10 terms of GO analysis and KEGG pathway analysis.

\section{Identification key genes related to immune-infiltration}

Among genes that are highly expressed in tumors, this study searched for genes which are closely related to high degree of immune-infiltration of two crucial immune cells. Also, the same method was used to identified the low expression key genes. R package "VennDiagram" was installed on R software to visualize these results. Receiver operating characteristic (ROC) curve of each key genes was used to assess the capability of distinguish form patients for healthy individuals according to areas under this curve and survival valve of each key genes.

\section{Construction of RUFY4 co-expression network and pathway cross-talk network}

The $c B i o$ Portal provides an online assistance for studying multidimensional cancer genomics data[20]. To construct the co-expression network of RUFY4, genes with a Spearman correlation index $>0.55$ are regarded to be closely related to the expression of RUFY4. Then, these genes were submitted to Cytoscape[21] to construct the pathway cross-talk network via a "Cluego" plug-in.

GSEA was performed by Windows desktop program v4.1.0 using MSigDBC2 Canonical pathways gene set collection. The GSEA result of RUFY4, was therefore conducted on all known genes ranked by enrichment score from most positive and most negative. 1000 random sample permutations were carried out.

\section{Verification of the Immune correlation and Prognostic Valve}

For verifying the immune correlation of the key gene, this study implemented two kinds of analysis to explore the relevance underlying them. The correlation index Person-r and Spearman-r and corresponding $p$-value are visualized via R package ggplot. To validity the relationship between RUFY4 and clinical attributes of ccRCC, clinical information, such as clinical stage, histopathology grade, TNM stage were analyzed by R package "readr" on R software. Statistically significant differences between two groups and among more than two groups were determined using Wilcox test and Kruskal test, respectively. By combining the expression level of RUFY4 and the known prognostic predictive factors such as age, gender, stage $T$, stage $M$, etc., univariate survival analysis was severed to verify the prognostic predictive effect of RUFY4.

\section{Results}




\section{Data source and data cleaning}

The clinical data and the RNA expression information of 611 samples were downloaded and then reorganized into three matrices, the clinical information of 537 patients, RNA-Seq of 611 samples in FPKM and RNA-Seq of 611 samples in COUNT. The whole analytical processes of this study were shown in a flow chart as Fig. 1.

\section{Establishment immune-infiltration landscape}

When the RNA-seq FKPM matrix coming from 611 ccRCC samples were screened by CIBERSORTx algorithm, the difference of immune-infiltration between ccRCC and normal tissues in 22 immune cells types were comprehensively tracked out. As shown in Fig. 2A, the proportions of 22 immune cells between ccRCC and control tissues were significantly different in several cell types. The heatmap of 22 immune cells were shown in Fig. 2B. In order to further examine the difference of immune-infiltration cell contents between ccRCC and control tissues, we pictured a violin plot to quantitatively describe the differences based on tumor tissue (537 samples) and adjacent normal tissues (72samples). According to the violin plot (Fig. 2C), B cells naïve, Plasma cells, T cells CD8, T cells CD4 naïve, T cells CD4 memory resting, $T$ cells CD4 memory activated, $T$ cells follicular helper (TFHs), $T$ cells regulatory (Tregs), $T$ cells gamma delta, Monocytes, Macrophages M0, Macrophages M1, Dendritic cells resting, Dendritic cells activated, Mast cells resting, Eosinophils and Neutrophils possessed difference abundance ratios and these differences are statistically significant. Immune cell types, such as T cells CD8, TFHs and Tregs, were obviously highly infiltrated in tumor tissue. These results showed that the proportions of immuneinfiltration cells might help us to distinguish ccRCC from normal individuals, and these cells might own some potential prognostic valve.

\section{Identifying Survival-associated Immune Cells}

In the last step, 17 immune cell types were obtained because of their difference of immune-infiltration between tumor and normal tissue. Here, the correlation between the proportions of 17 types of immune cell and OS rates were analyzed via Kaplan-Meier (KM) curves. The outcome in Figs. 3A-H showed that the fractions of 8 immune cells were tied to overall survival rates, including T cells CD 4 memory activated, T cells CD4 memory resting, T cells CD8, Macrophages M0, Macrophages M1, Tregs, TFHs and Neutrophils. Notably, TFHs and Tregs had the two highest hazard ratio (HR) values of 1.62 and 1.58, respectively, while T cells CD4 memory resting and Neutrophils were negatively correlated with overall survival. Figures 31 showed that though activated mast cell had no statistical difference in relative proportions between tumor and normal tissue, it also had a disjoint K-M curve.

\section{Relationship between clinical attributes and survival- associated immune cells}


To further understand the clinical predictive value of survival-associated cells, the spearman correlation analysis was conducted between the contents of the 8 survival- associated immune cells and clinical attributes. As shown in Fig. 4A-F and Supplementary Fig. 1A-B, the proportions of Macrophages M0, T cells CD8, TFHs and Tregs climbed with the increase of histopathological grade, while the fractions of $T$ cells CD4 memory resting decreased in an opposite manner. As for clinical stage, it was divided into two categories based on the common knowledge. Early-stage contains stage I, stage II and stage III, while late-stage only includes stage IV. As presented in Fig. 4G-I, higher proportions of T cells CD8, TFHs and Tregs was closely related to late-stage tumor and the difference was significant statistically. As for the analysis of stage $T$, stage $\mathrm{N}$, stage $\mathrm{M}$, which were shown in Fig. 4J-P, identical results of $\mathrm{T}$ cells $\mathrm{CD} 8$, TFHs and Tregs were founded. These three types of immune cells always heralded more advanced tumors in ccRCC patients. Remarkably, T cells CD4 memory resting often stood on the opposite side of the above three cells, and its increased proportions indicated early-stage tumors (Supplementary Fig. 1C). Therefore, these cells held much appeal for this study.

\section{Screening crucial survival-associated immune cells in TME}

In order to narrow down our target immune cells in TME, a spearman correlation analysis was performed in the above 8 kinds of immune cell. As shown in Fig. 5A, T cells CD4 memory resting were negatively correlated with T cells CD8, TFHs and Tregs. These results were consistent with the previous results. Notably, TFHs and Tregs were correlated with each other and they both had correlation indices higher than 0.3 with serval cells, including T cells CD8. These results suggested that cross-talks between these cells existed indeed.

Multivariate survival analysis was conducted to assess the prognostic value of the above 8 immune cells and clinicopathologic parameters was chosen as OS rates (Table 1) for these patients with ccRCC. The abundance ratios of TFHs and Tregs were independent prognostic factors for OS (Fig. 5B-C), after consideration for known risk factors such as age, clinical stage, $\mathrm{T}$ stage, $\mathrm{M}$ stage, and histopathological grade.

Survival analyses reflected that higher immune-infiltration of TFHs or Tregs was associated with inferior OS. Overall survival curves according to TFHs or Tregs proportions in cCRCC tumor tissues were distinctly disjoined. Further analysis of the multivariate survival analysis model found that the ratio of TFHs and Tregs could not be put into the model at the same time. Once TFHs or Tregs were together put into the model for analysis as known prognostic factors, one of them was bound to be non-statistically significant. Based on statistical theories, this study inferred that there was a strong interaction between TFHs or Tregs, which was consistent with the previous correlation analysis results. Taken together, these observations suggested that TFHs or Tregs were two crucial immune cells in tumor microenvironment of ccRCC patients.

\section{Identification and Enrichment Analysis of IIRGs}


This study obtained 676 genes had significant different expression levels between high and low proportions of Tregs in TME, 353 of which possessed much higher expression. As for TFHs, 680 different expression genes were found while 620 genes were related to higher immune-infiltration. These observations were presented in Fig. 6A and Fig. 6B. After uploading these genes to the website DAVID, top 10 items of TFHs in Gene Ontology (GO) analysis and Kyoto Encyclopedia of Genes and Genomes (KEGG) analysis results were shown in Fig. 6D-G. Biological process of GO analysis (Fig. 6D) reflected that TFHs might involve in cellular protein metabolic process and cell communication while Cellular Component (Fig. 6G) showed that these genes were enriched in extracellular exosome and plasma membrane. Molecular function of these genes (Fig. 6E) was gathered in serine-type endopeptidase activity, antigen binding and sequence-specific DNA binding. KEGG analysis (Fig. 6F) suggested that cytokine-cytokine receptor interaction was the key and dominant pathway. As for Tregs (Supplementary Fig. 1D-G), cellular protein metabolic process at biological process level, extracellular exosome at cellular component level, serine-type endopeptidase activity at molecular function level were the most crucial enrichment items. The results of KEGG pathway analysis greatly were the same as the results of Tregs. To make a summary, the results of enrichment analysis showed that the two crucial immune-associated cells had a large degree of overlapped function, which meant that the two cells might participate in the same biological process, and that there might be cross-talks underlying the TME.

\section{Identification key genes related to immune-infiltration}

Three gene sets, including genes related to high immune-infiltration of Tregs, genes related to high immune-related of TFHs and genes highly expressed in tumor tissues were submitted to Packages "VennDiagram" on R software. As shown in Fig. 7A, SLC12A1 was the sole gene which was closely related to low degree of immune-infiltration of the two crucial immune cells, and it was also silenced in tumor tissues. Remarkably, when narrowing the search scope to genes that were high expressed in tumor tissue, LAG3, PDCD1 and RUFY4 were found to be associated to the high degree of immune-infiltration of Tregs and TFHs. Form the Fig. 7B, it could be observed that 94 genes were not only connected with the increased immune-infiltration of Tregs, but also inseparable from the degree of immune-infiltration of TFHs. The ROC results of the above 4 key genes, including one downregulated gene SLC12A1 and 3 upregulated genes LAG3, PDCD1 and RUFY4, were presented in Fig. 7C-F. RUFY4 had a relatively bigger AUC (area under the curve) than the other key genes, suggesting that it had a more accurate diagnostic value for ccRCC. So far, this study had found a key gene, which was in the TME related to the high immune-infiltration of two crucial cells, TFHs and Tregs. At the same time, RUFY4 also had an activated expression in tumor tissues, making it have accurate and sensitive distinctions ability between normal and ccRCC patients.

\section{Construction of RUFY4 co-expression network and immune signaling network}


From the website cBioPortal, 493 genes were mined to construct a co-expression network, which not only contained 492 positively related genes, but also contained one negatively related gene "ITGA6". In order to verify the accuracy and reproducibility of the co-expression network construction, this study selected genes with an absolute value of spearman correlation coefficient greater than 0.7 on the website, and reverified mutual correlations with the TCGA RNA-FPKM matrix constructed in the first step. The results shown in Fig. 8A denoted that the co-expression network created on the website was trustworthy and indicated that there was a potential regulatory mechanism for RUFY4. As shown in the Fig. 8B, these genes closely associated to RUFY4 were involved in several crucial biological processes, two categories of which appealed to this study. The first one was inseparably related to regulation of immune cells and molecules, including cytokine-cytokine receptor interaction, PD-L1 (Programmed cell death 1 ligand 1) expression and PD-1 (Programmed death-1) checkpoint pathway in cancer, T helper cells differentiation, T cell receptor signaling pathway and primary immunodeficiency; the second category consisted of two signal transduction pathways, JAK-STAT (Janus kinase 2-signal transducer and activator of transcription 3) and NF-kappa B (Nuclear factor kappa beta). These results emphasized that the co-expression network of RUFY4 in cCRCC might have an inseparable connection with immunomodulation and tumor immunotherapy, indicating that RUFY4 owned the potential research value as a biomarker.

When choosing Canonical Pathways gene sets derived from the KEGG pathway database as the molecular signatures database (Fig. 8C-D), gene set enrichment analysis (GSEA) indicated that RUFY4 was highly associated with $T$ cell receptor signaling pathway and cytokine-cytokine receptor interaction. Moreover, as presented in Fig. 8E-F, it was found that when compared with gene sets derived from the WikiPathways pathway database, cancer-immunotherapy by PD1-blockade and costimulatory signaling of $T$ cell receptor were the two most significant enrichment pathways, whose q-FDR values both equaled to zero. These evidences indicated that the high expression of RUFY4 was inseparable from the two biological processes. So far, this study had investigated through public databases that RYFU4 might regulate the function of immune cells in TME via the interaction of cytokines and participate in the process of tumor immunosuppression.

\section{Clinical and immune applications of RUFY4}

From the Fig. 9A-B, it could be demonstrated that there were correlations between half of the 22 types of immune cells and RUFY4, 5 types of which were positively correlated with RUFY4 while 6 were negatively correlated. Consistent with the previous conclusions of this study, a significantly stronger relationship between RUFY4 and T cells CD8, TFH, Tregs than other cell types could be easily dug up. Next, this study determined the correlation between RUFY4

and ccRCC clinical attributes. RUFY4's expression levels were consistently correlated with ccRCC clinical stage (Fig. 9C), histopathological grade (Fig. 9D) and T stage (Fig. 9E). These results, together with the finding that mRNA levels of RUFY4 in ccRCC patients were strongly correlated with the metastasis of distant organs (Fig. 9F) and regional lymph nodes (Fig. 9G) predicted the unfavorable prognosis of cCRCC. 
Multivariate survival analysis was implemented to assess the prognostic value of RUFY4's expression levels for these patients with ccRCC. The results showed that RUFY4 were an independent prognostic factor for OS, even after adjusting known factors, such as clinical stage, $M$ stage, and histopathological grade. Survival analysis revealed that the increasing expression of RUFY4 was associated with inferior OS, whose curve according to RUFY4 expression in ccRCC tissues were distinctly disjoined (Fig. 9H). Taken together, these observations suggested that RUFY4 expression might contributes to progression of ccRCC as a prognostic biomarker.

\section{Discussion}

Clear cell RCC $a$ is one of the most lethal types of urogenital tumors worldwide with an increasing mortality rate over several years[22].

Since Galon J.[23] first proposed the concept of immune contexture in 2007, researches associated with the location, density and functional orientation of different immune cells in tumor microenvironment have put immunotherapy into the cutting-edge frontier. Among the current therapeutic strategies, ICI have become a key option for various cancer types[24]. Ample clinical trials show that PD-1 blockade is a vital method in management of $\mathrm{CCRCC}$, and therapies based on anti-PD-1 are the first-line chooses for refractory patients[25,26]. The aim of this study was to figure out immune cells and genes closely associated to both immune-infiltration and prognosis in TME. This study not only determined immuneinfiltration cells and gene signatures for ccRCC immunotherapy, but also proposed a new research method for exploring potential regulation targets in TME.

In this study, eight types of immune cells were considered as dramatic association with ccRCC patients' survival, including T cells CD4 memory activated, T cells CD4 memory resting, $T$ cells CD8, Macrophages M0, Macrophages M1, Tregs, TFHs and Neutrophils. Among the aforementioned immune cells, T cells CD8 and T cells CD4 memory resting had absolute advantages in content, which were much higher than other cell types. Currently, T cell CD8 is a popular topic in tumor immunotherapy. The PD-L1 produced by tumor tissue binds to the PD-1 located on the outer membrane of T cells CD8 to limit the anti-tumor immune response. Theoretically, PD-L1 inhibitor induces the accumulation of T cells CD8, resulting in an effective anti-tumor immune response. However, Braun D.A. proposed that baseline T cells CD8 infiltration does not predict response to PD-1 blockade[27]. As for T cells CD4 memory resting, there is another name, central memory $T$ cells $C D 4$, which are distributed in lymphoid tissues with a static state, typically express CD62L and CCR7[28]. T cells CD4 memory resting maintain immune response memory and presents immunoprotected effects when tumors begin metastasizing. Furthermore, together with T cells CD4 memory activated, they are involved in multiple processes of various cancers, such as lymph node metastasis of breast cancer cells[29], progression of lung cancer[30] and drug resistance of pancreatic cancer[31]. In this study, patients with denser T cells CD4 memory resting had longer overall survival and presented more vicious tumors with senior TNM stage, while patients with higher T cells CD8 had shorter overall survival. These findings are consistent with the theory of T cell depletion. 
Among the immune cell types estimated, only TFHs and Tregs were still related to patient's overall survival after adjusting the known risk factors in multivariate survival analysis. TFHs are specialized T helper $(\mathrm{TH})$ cells and different from other subgroups such as $\mathrm{TH} 1, \mathrm{TH} 2$ and $\mathrm{TH} 17$. It is widely accepted that TFH-B cell axis in the tumor-associated tertiary lymphoid structure favorably forms an immune environment with antitumor effects[32]. Large number of TFHs predict improved survival in breast cancer[33] and are associated with positive prognosis in colorectal cancer[34]. On the contrary, TFHs predicts negative clinical prognostic in lung squamous cell carcinoma[35]. These results put TFHs in a controversial position where further exploration is needed to determine whether it is a protective factor or a risk factor. Another crucial survival-associated immune cell is Treg, which is a widely-known restrainer of immune regulatory network. The expression of the chief regulatory transcription factor FOXP3 is one of their characteristics and Tregs are involved in keeping the balance of immune responses[36]. They act as a double-edged sword. On the one hand, they can restrain unnecessary immune activation, such as autoimmunity or immunological rejection. On the other hand, useless immune responses are exerted by TREGs for pathogens or cancer cells, resting in progression of cancer[37]. In kidney cancers, there have been several evidences for the association between Tregs and poor prognosis and connections between Tregs and immunotherapy resistance. However, the mechanisms underlying the heterogeneity of Tregs is unclear and needed further exploration. To make a summary, two cells with the highest immuneinfiltration content and two crucial survival-associated cells were dug out in this study, and all of them play important roles in constructions of TME and immunotherapy of patients, which indicates that the next analysis based on these cells is reliable and convincing.

Further, Sato, E. found that an increased ratio of T cells CD8 to Tregs in tumors was associated to favorable clinical outcome of patients with ovarian cancer and suggested that effector T cells CD8 was suppressed by Tregs[38]. Additionally, Tregs in breast cancer can eliminated T cells CD8 cytotoxicity synergistically induced by Interleukin-2 (IL-2) and IL-10[39]. Referring to the crosstalk between THFs and $T$ cells CD8, THFs effectively enhance the effector function of T cells CD8 with an IL-21-dependent manner in colorectal cancer[40]. And researches conducted by Chu, F. has determined that T cells CD8 own abilities to inhibit TFHs-mediated B cell differentiation, and exert powerful anti-tumor activity to bring favorable prognosis for patients with follicular lymphoma[41]. These results make the regulatory network including T cells CD8, THFs and Tregs extremely complicated. In order to explore the potential interaction of TFHs and Tregs on T cells CD8, we have performed functional enrichment of genes associated with these cells. The functional enrichment analysis showed that TFHs and Tregs take apart in the same biological process, such as cytokine-cytokine receptor interaction. More recently, like IL-1, IL-33, cytokines are the central mediators of cell-cell interaction. By regulating different components of TME, including recruiting tumor infiltrating myeloid cells, angiogenesis, and inhibiting anti-tumor immunity, cytokines have become key components to promote tumor progression[42-44]. Therefore, this study predicts that TFHs and Tregs have great impact on functions of T cells CD8 through the interaction between cytokines secreted by this two cells and cytokine receptors on the surface of T cells CD8, which in turn affects CD 8 involvement in the development and progression of $\mathrm{cCRCC}$, and may influence the treatment effect of ICI in cCRCC. 
Among all IIRGs in this study, RUFY4 is the only one that satisfies the following five conditions at the same time, a) it is highly expressed in ccRCC tumor tissues; b) it is closely related to the dense infiltration of TFHs; c) it is closely related with highly infiltrated TREGs, d) it has extremely high specificity and sensitivity to distinguish between healthy persons and ccRCC patients; e) it is obviously related to the prognosis of patients. Although the importance of IIRGs in tumor progression and immunotherapy has been recognized, there are few studies dedicated to genes related to the immune-infiltration of two specific cell types, TFHs and Tregs. Therefore, this research innovatively proposes a method of targeted selection of IIRGs. Next, of the functional analysis carried on CYTOSCAPE, we found that RUFY4 was also involved in cytokine-cytokine receptor interaction which has been already discussed in last section. Among the other functional analysis results, RUFY4 was closely tied to two signal transduction pathways, JAK-STAT and NF-kappa B. In lung cancers, the positive rate of PD-L1 is significantly increased with the accumulation JAK3 mutation, Therefore, some patients may benefit from anti-PD-1 treatment because of JAK3 germline activating mutations[45]. Also the downstream JAK-STAT of IL-1 activates to generate inflammatory fibroblasts to promote treatment resistance in pancreatic ductal carcinoma[46]. Not only that, in cCRCC, the JAK-STSA family is also related to the mutation of PBRM1, which indirectly participates in the regulation of $\mathrm{ICI}$ therapy[47]. When referring to NF-kappa B signaling pathway, its role and function in TME cannot be ignored and is worthy to be mentioned. Under physiological conditions, the NF-kappa B is limited by negative feedback, but this self-regulation is often out of control in cancer cells leading to promotes vitality, angiogenesis, and proliferation of tumor cell. NF-kappa B can also affect other cells in the TME by inducing the secretion of cytokines[48]. Therefore, NF-kappa B acts as a tumor promoter by regulating the function of infiltrating lymphocytes and macrophages to affect the body innate immune response to cancer cells[49]. In ccRCC, the expression of NF-Kappa B correlates with apoptosis and angiogenesis in cCRCC tissues. Based on these results, we found that RUFY4, which had a certain predictive value for the poor prognosis of patients, might play role in the regulation of TME and the production of immunotherapy resistance through the aforesaid two signaling pathways.

In addition to the above JAK-STAT and NF-kappa B signaling pathways, the most noticeable concerns in the single-gene GSEA analysis results of RUFY4 are the two kinds of receptor, cytokine receptor and PD-1. TFHs and Tregs are related to the cytokine-cytokine signaling pathway, which has been mentioned above. It is quite reasonable and acceptable that our target gene RUFY4 selected based on TFHs and Tregs is also related to cytokine-cytokine signaling, and these consistent results suggest that RUFY4 may participate in TFHs and Tregs exercise of regulatory functions through the interaction between cytokines. Through the previous discussion of this study and the existing consensus, we have learned Treg is the main immune negative regulatory cell in TME. More than that, PD-L1 on the Treg surface inhibits the antitumor activity of PD-1 of CD8 T cells. This process is a two-way antagonistic process, in which Treg secretes TGF- $\beta$, IL-10 and other cytokines to inhibit the anti-tumor immune response and PD-1 on the surface of lymphocytes promotes and amplifies the secretion of TGF- $\beta[50]$. Therefore, the persistently high expression of PD-1 in TME can not only promote the generation of Tregs but also continue to inhibit the anti-tumor effect of the cell. Not only are TREG cells closely related to the PD-1 pathway, but TFH cells themselves also function through the PD-1 pathway. In osteosarcoma and rectal cancer patients whose 
tumor cells express large quantities of PD-L1s, the PD-1 and PD-L1 pathway on TFHs can be bound by tumor cells PD-L1 to mediate immunosuppression, thereby significantly reducing the IL-21 produced by TFHs[51]. However, if supplemented with anti-PD-L1 antibody, the anti-tumor response of TFHs can be recused[40]. So far, the signaling pathways closely related to RUFY4 are involved in the formation of the regulatory network of TFHs and Tregs, from which there are also regulatory function on the interaction between PD-1 on T cells CD8 and PD-L1 of the other cells in TME.

\section{Conclusion}

This present study analyzed and verified a unique gene (RUFY4) closely related to immune-infiltration of TFHs and Tregs. These cells and gene are separately recognized as independent prognostic biomarkers of ccRCC patients. Moreover, RUFY4 are associated to the formation and regulation of an immune network in TME, thus reflecting the function of RUFY4 in predicting the responsiveness of ICI therapy. Hopefully, our findings provide evidences and strategies for digging out effective signatures of clinical outcome and therapeutic targets for patients.

\section{Abbreviations}

IIRG: immune-infiltrated-related gene

TFHs: T cells follicular helper

Tregs: T cells regulatory

RUFY: RUN and FYVE domain-containing protein

PI3P: Phosphatidylinositol 3-phosphate

PODXL: podocalyxin-like

TCGA: The Cancer Genome Atlas Project

FPKM: Fragments Per Kilobase of exon model per Million mapped fragments

KM: Kaplan-Meier

GO: Gene Ontology

KEGG: Kyoto Encyclopedia of Genes and Genomes

PD-L1: Programmed cell death 1 ligand 1

PD-1: Programmed death-1

ccRCC: clear cell Renal cell carcinoma 
ICI: immune checkpoint inhibitors

TME: tumor microenvironment

\section{Declarations}

\section{Ethics approval and consent to participate}

Not applicable.

\section{Consent for publication}

All participants agreed to publish this article.

\section{Availability of data and materials}

The datasets generated analyzed during the current study are available in the TCGA repository (https://portal.gdc.cancer.gov).

\section{Competing interests}

There is no any conflict of interest.

\section{Funding}

This study was supported by the National Natural Science Foundation of China (81972630, 81902588, 81874090), the National Key Scientific Instrument Development Project (81927807), National Key R\&D Program of China (2017YFB1303100), Wuhan Science and Technology Plan Application Foundation Frontier Project (2020020601012247).

\section{Authors' contributions}

YHM and ZXP designed and directed data processing procedures. SJ, XZY, YCF, XW, MGX, CZX, LQY and $X K R$ analyzed the data. MDJ wrote the manuscript. All authors approved the final manuscript.

\section{Acknowledgements}

Not applicable. 


\section{References}

1. Bray, F., et al., Global cancer statistics 2018: GLOBOCAN estimates of incidence and mortality worldwide for 36 cancers in 185 countries. CA: a cancer journal for clinicians, 2018. 68(6): p. 394424.

2. Hsieh, J.J., et al., Renal cell carcinoma. Nat Rev Dis Primers, 2017. 3: p. 17009.

3. Choueiri, T.K. and R.J. Motzer, Systemic Therapy for Metastatic Renal-Cell Carcinoma. N Engl J Med, 2017. 376(4): p. 354-366.

4. Hanahan, D. and R.A. Weinberg, Hallmarks of cancer: the next generation. Cell, 2011. 144(5): p. 64674.

5. Fridman, W.H., et al., The immune contexture in human tumours: impact on clinical outcome. Nat Rev Cancer, 2012. 12(4): p. 298-306.

6. Yoshihara, K., et al., Inferring tumour purity and stromal and immune cell admixture from expression data. Nature communications, 2013. 4: p. 2612.

7. Şenbabaoğlu, Y., et al., Tumor immune microenvironment characterization in clear cell renal cell carcinoma identifies prognostic and immunotherapeutically relevant messenger $R N A$ signatures. Genome biology, 2016. 17(1): p. 231.

8. Ueda, K., et al., Prognostic value of PD-1 and PD-L 1 expression in patients with metastatic clear cell renal cell carcinoma. Urologic oncology, 2018. 36(11).

9. Mikami, S., et al., Clinical significance of programmed death-1 and programmed death-ligand 1 expression in the tumor microenvironment of clear cell renal cell carcinoma. Cancer science, 2019. 110(6): p. 1820-1828.

10. Char, R. and P. Pierre, The RUFYs, a Family of Effector Proteins Involved in Intracellular Trafficking and Cytoskeleton Dynamics. Frontiers in cell and developmental biology, 2020. 8: p. 779.

11. Taniuchi, K., et al., Measurement of serum PODXL concentration for detection of pancreatic cancer. OncoTargets and therapy, 2018. 11: p. 1433-1445.

12. He, S., et al., PODXL might be a new prognostic biomarker in various cancers: a meta-analysis and sequential verification with TCGA datasets. BMC cancer, 2020. 20(1): p. 620.

13. Shin, N., et al., Identification of frequently mutated genes with relevance to nonsense mediated mRNA decay in the high microsatellite instability cancers. International journal of cancer, 2011. 128(12): p. 2872-2880.

14. Guseva, N.V., et al., Anchored multiplex PCR for targeted next-generation sequencing reveals recurrent and novel USP6 fusions and upregulation of USP6 expression in aneurysmal bone cyst. Genes, chromosomes \& cancer, 2017. 56(4): p. 266-277.

15. Staubitz, J.I., et al., Novel rearrangements involving the RET gene in papillary thyroid carcinoma. Cancer genetics, 2019. 230: p. 13-20.

16. Men, W., et al., RUFY3 Predicts Poor Prognosis and Promotes Metastasis through Epithelialmesenchymal Transition in Lung Adenocarcinoma. Journal of Cancer, 2019. 10(25): p. 6278-6285. 
17. Xie, R., et al., RUFY3 interaction with FOXK1 promotes invasion and metastasis in colorectal cancer. Scientific reports, 2017. 7(1): p. 3709.

18. Terawaki, S., et al., RUN and FYVE domain-containing protein 4 enhances autophagy and lysosome tethering in response to Interleukin-4. The Journal of cell biology, 2015. 210(7): p. 1133-1152.

19. Newman, A.M., et al., Determining cell type abundance and expression from bulk tissues with digital cytometry. Nature biotechnology, 2019. 37(7): p. 773-782.

20. Gao, J., et al., Integrative analysis of complex cancer genomics and clinical profiles using the cBioPortal. Science signaling, 2013. 6(269): p. pl1.

21. Shannon, P., et al., Cytoscape: a software environment for integrated models of biomolecular interaction networks. Genome research, 2003. 13(11): p. 2498-2504.

22. Rini, B.I., S.C. Campbell, and B. Escudier, Renal cell carcinoma. Lancet (London, England), 2009. 373(9669): p. 1119-1132.

23. Galon, J., W.-H. Fridman, and F. Pagès, The adaptive immunologic microenvironment in colorectal cancer: a novel perspective. Cancer research, 2007. 67(5): p. 1883-1886.

24. Ribas, A. and J.D. Wolchok, Cancer immunotherapy using checkpoint blockade. Science (New York, N.Y.), 2018. 359(6382): p. 1350-1355.

25. Motzer, R.J., et al., Nivolumab plus Ipilimumab versus Sunitinib in Advanced Renal-Cell Carcinoma. The New England journal of medicine, 2018. 378(14): p. 1277-1290.

26. Rini, B.I., et al., Pembrolizumab plus Axitinib versus Sunitinib for Advanced Renal-Cell Carcinoma. The New England journal of medicine, 2019. 380(12): p. 1116-1127.

27. Braun, D.A., et al., Interplay of somatic alterations and immune infiltration modulates response to PD1 blockade in advanced clear cell renal cell carcinoma. Nat Med, 2020. 26(6): p. 909-918.

28. Pepper, M. and M.K. Jenkins, Origins of CD4(+) effector and central memory T cells. Nature immunology, 2011. 12(6): p. 467-471.

29. MacLeod, M.K.L., J.W. Kappler, and P. Marrack, Memory CD4 T cells: generation, reactivation and reassignment. Immunology, 2010. 130(1): p. 10-15.

30. Voigt, C., et al., Cancer cells induce interleukin-22 production from memory CD4 T cells via interleukin1 to promote tumor growth. Proceedings of the National Academy of Sciences of the United States of America, 2017. 114(49): p. 12994-12999.

31. Gu, J., et al., Activating miRNA-mRNA network in gemcitabine-resistant pancreatic cancer cell associates with alteration of memory CD4 T cells. Annals of translational medicine, 2020. 8(6): p. 279.

32. Bruni, D., H.K. Angell, and J. Galon, The immune contexture and Immunoscore in cancer prognosis and therapeutic efficacy. Nature reviews. Cancer, 2020. 20(11): p. 662-680.

33. Gu-Trantien, C., et al., CD4囚 follicular helper $T$ cell infiltration predicts breast cancer survival. The Journal of clinical investigation, 2013. 123(7): p. 2873-2892. 
34. Bindea, G., et al., Spatiotemporal dynamics of intratumoral immune cells reveal the immune landscape in human cancer. Immunity, 2013. 39(4): p. 782-795.

35. Xu, F., et al., Immune signature of $T$ follicular helper cells predicts clinical prognostic and therapeutic impact in lung squamous cell carcinoma. International immunopharmacology, 2020. 81: p. 105932.

36. Lakkis, F.G., Immunology: TREG cells in transplantation-a double-edged sword? Nature reviews. Nephrology, 2014. 10(4): p. 185-186.

37. Wolf, D., et al., Treg(s) in Cancer: Friends or Foe? Journal of cellular physiology, 2015. 230(11): p. 2598-2605.

38. Sato, E., et al., Intraepithelial CD8+ tumor-infiltrating lymphocytes and a high $C D 8+/$ regulatory $T$ cell ratio are associated with favorable prognosis in ovarian cancer. Proceedings of the National Academy of Sciences of the United States of America, 2005. 102(51): p. 18538-18543.

39. Li, X., et al., Interleukin 2 and interleukin 10 function synergistically to promote CD8 T cell cytotoxicity, which is suppressed by regulatory $T$ cells in breast cancer. The international journal of biochemistry \& cell biology, 2017. 87: p. 1-7.

40. Shi, W., et al., Follicular helper T cells promote the effector functions of CD8 T cells via the provision of IL-21, which is downregulated due to PD-1/PD-L1-mediated suppression in colorectal cancer. Experimental cell research, 2018. 372(1): p. 35-42.

41. Chu, F., et al., CXCR5CD8 T cells are a distinct functional subset with an antitumor activity. Leukemia, 2019. 33(11): p. 2640-2653.

42. Malik, A. and T.-D. Kanneganti, Function and regulation of IL-1a in inflammatory diseases and cancer. Immunological reviews, 2018. 281(1): p. 124-137.

43. Ilyin, S.E., et al., Autoregulation of the interleukin-1 system and cytokine-cytokine interactions in primary human astrocytoma cells. Brain research bulletin, 2000. 51(1): p. 29-34.

44. Afferni, C., et al., The Pleiotropic Immunomodulatory Functions of IL-33 and Its Implications in Tumor Immunity. Frontiers in immunology, 2018. 9: p. 2601.

45. Li, S.D., et al., Cancer gene profiling in non-small cell lung cancers reveals activating mutations in JAK2 and JAK3 with therapeutic implications. Genome medicine, 2017. 9(1): p. 89.

46. Biffi, G., et al., IL 1-Induced JAK/STAT Signaling Is Antagonized by TGF $\beta$ to Shape CAF Heterogeneity in Pancreatic Ductal Adenocarcinoma. Cancer discovery, 2019. 9(2): p. 282-301.

47. Miao, D., et al., Genomic correlates of response to immune checkpoint therapies in clear cell renal cell carcinoma. Science (New York, N.Y.), 2018. 359(6377): p. 801-806.

48. Bradford, J.W. and A.S. Baldwin, IKK/nuclear factor-kappaB and oncogenesis: roles in tumorinitiating cells and in the tumor microenvironment. Advances in cancer research, 2014. 121: p. 125145.

49. Taniguchi, K. and M. Karin, NF-KB, inflammation, immunity and cancer: coming of age. Nature reviews. Immunology, 2018. 18(5): p. 309-324. 
50. Gianchecchi, E. and A. Fierabracci, Inhibitory Receptors and Pathways of Lymphocytes: The Role of PD-1 in Treg Development and Their Involvement in Autoimmunity Onset and Cancer Progression. Frontiers in immunology, 2018. 9: p. 2374.

51. Gao, W., J. Zhou, and B. Ji, Evidence of Interleukin 21 Reduction in Osteosarcoma Patients Due to PD1/PD-L 1-Mediated Suppression of Follicular Helper T Cell Functionality. DNA and cell biology, 2017. 36(9): p. 794-800.

\section{Table}

Due to technical limitations, table 1 is only available as a download in the Supplemental Files section.

\section{Figures}

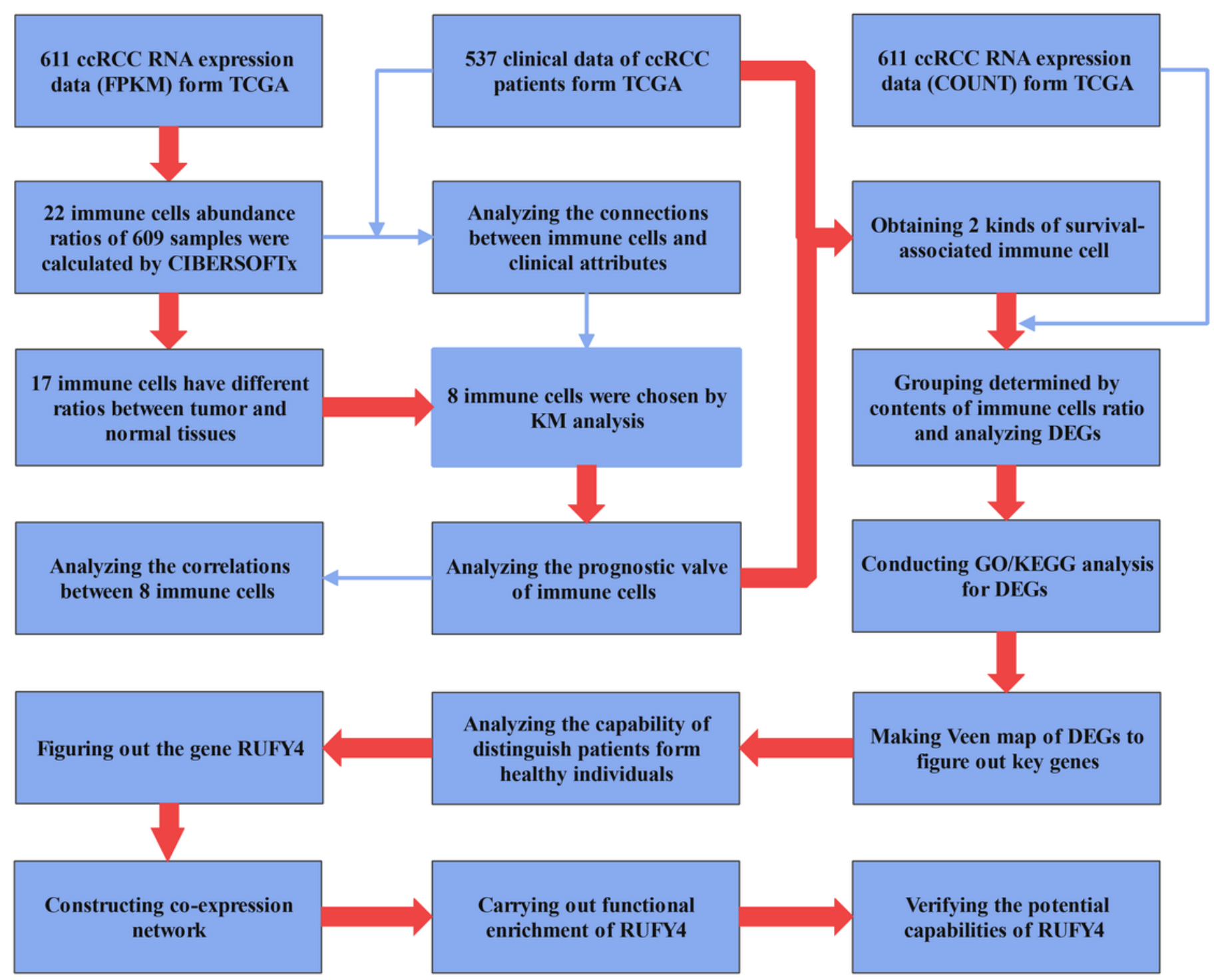


The data processing flowchart of this study FRKM and COUNT are two types of RNA sequencing data downloaded from TCGA database. CIBERSORTx is an online tool to calculated the abundance ratios of immune cells in mixed samples by using RNA expression information. Kaplan-Meier (KM) curve is a univariate survival analysis method to preliminary determine prognostic value of immune cells. Multivariate survival analysis clarified the prognostic value of two kinds of immune cell. DEGs means differentially expressed genes; GO (Gene Ontology) and KEGG (Kyoto Encyclopedia of Genes, and Genomes) are functional enrichment analyses conducted on a website (https://david.ncifcrf.gov/). The co-expression network was composed of genes related to RUFY4 (correlation coefficient $>0.55$ ) and itself.

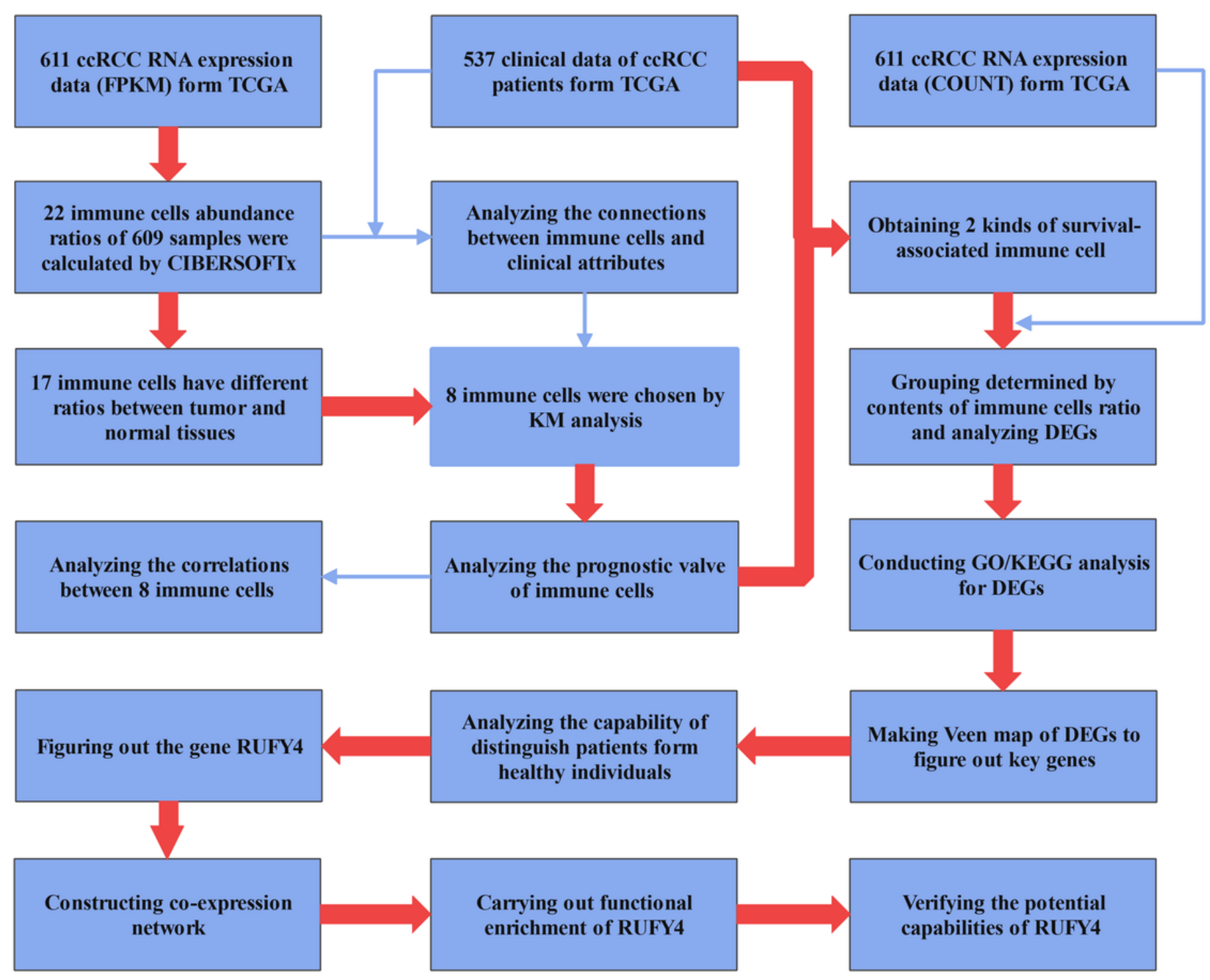

\section{Figure 1}

The data processing flowchart of this study FRKM and COUNT are two types of RNA sequencing data downloaded from TCGA database. CIBERSORTx is an online tool to calculated the abundance ratios of immune cells in mixed samples by using RNA expression information. Kaplan-Meier (KM) curve is a univariate survival analysis method to preliminary determine prognostic value of immune cells. 
Multivariate survival analysis clarified the prognostic value of two kinds of immune cell. DEGs means differentially expressed genes; GO (Gene Ontology) and KEGG (Kyoto Encyclopedia of Genes, and Genomes) are functional enrichment analyses conducted on a website (https://david.ncifcrf.gov/). The co-expression network was composed of genes related to RUFY4 (correlation coefficient $>0.55$ ) and itself.

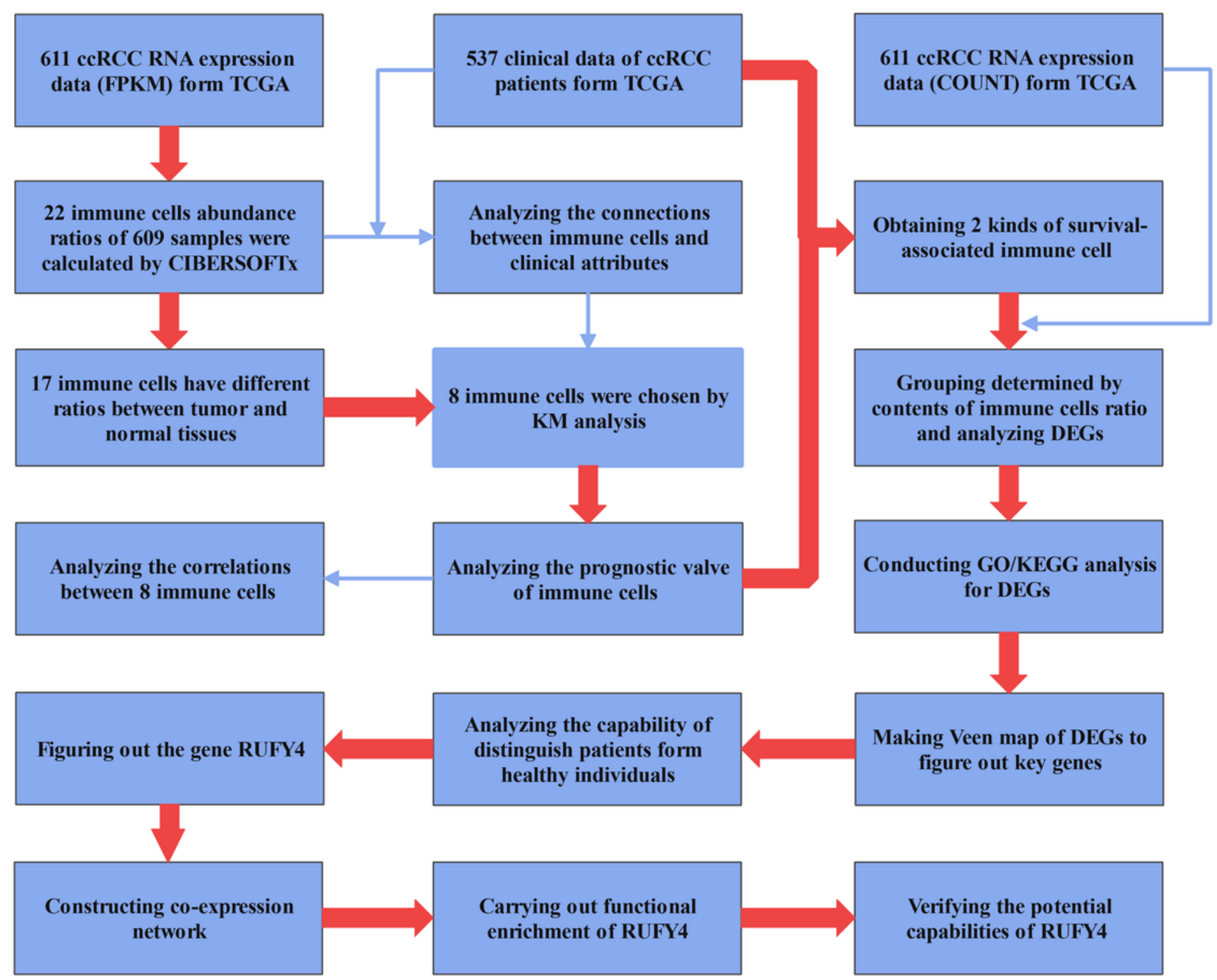

\section{Figure 1}

The data processing flowchart of this study FRKM and COUNT are two types of RNA sequencing data downloaded from TCGA database. CIBERSORTx is an online tool to calculated the abundance ratios of immune cells in mixed samples by using RNA expression information. Kaplan-Meier (KM) curve is a univariate survival analysis method to preliminary determine prognostic value of immune cells. Multivariate survival analysis clarified the prognostic value of two kinds of immune cell. DEGs means differentially expressed genes; GO (Gene Ontology) and KEGG (Kyoto Encyclopedia of Genes, and Genomes) are functional enrichment analyses conducted on a website (https://david.ncifcrf.gov/). The co-expression network was composed of genes related to RUFY4 (correlation coefficient $>0.55$ ) and itself. 


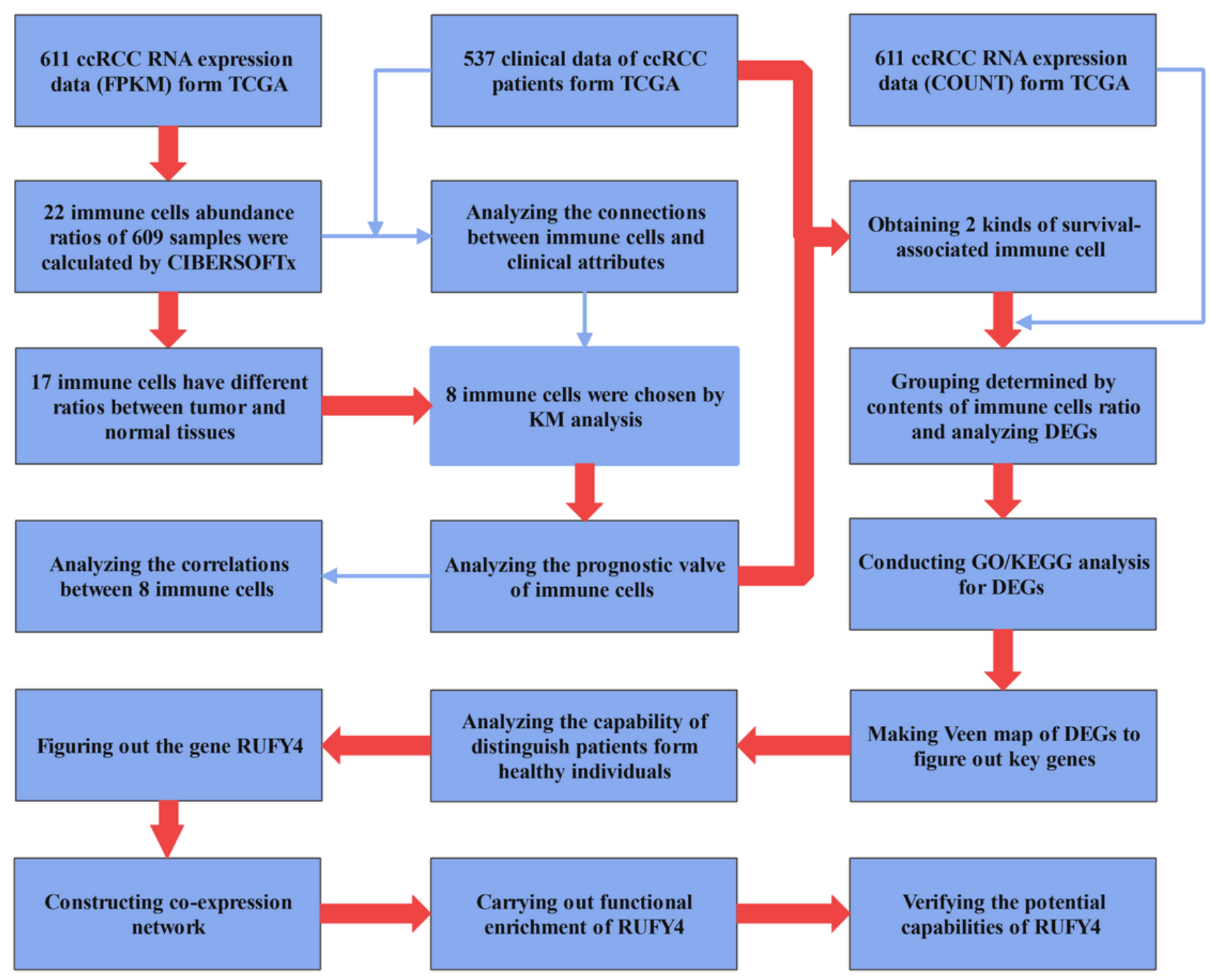

\section{Figure 1}

The data processing flowchart of this study FRKM and COUNT are two types of RNA sequencing data downloaded from TCGA database. CIBERSORTx is an online tool to calculated the abundance ratios of immune cells in mixed samples by using RNA expression information. Kaplan-Meier (KM) curve is a univariate survival analysis method to preliminary determine prognostic value of immune cells. Multivariate survival analysis clarified the prognostic value of two kinds of immune cell. DEGs means differentially expressed genes; GO (Gene Ontology) and KEGG (Kyoto Encyclopedia of Genes, and Genomes) are functional enrichment analyses conducted on a website (https://david.ncifcrf.gov/). The co-expression network was composed of genes related to RUFY4 (correlation coefficient>0.55) and itself. 

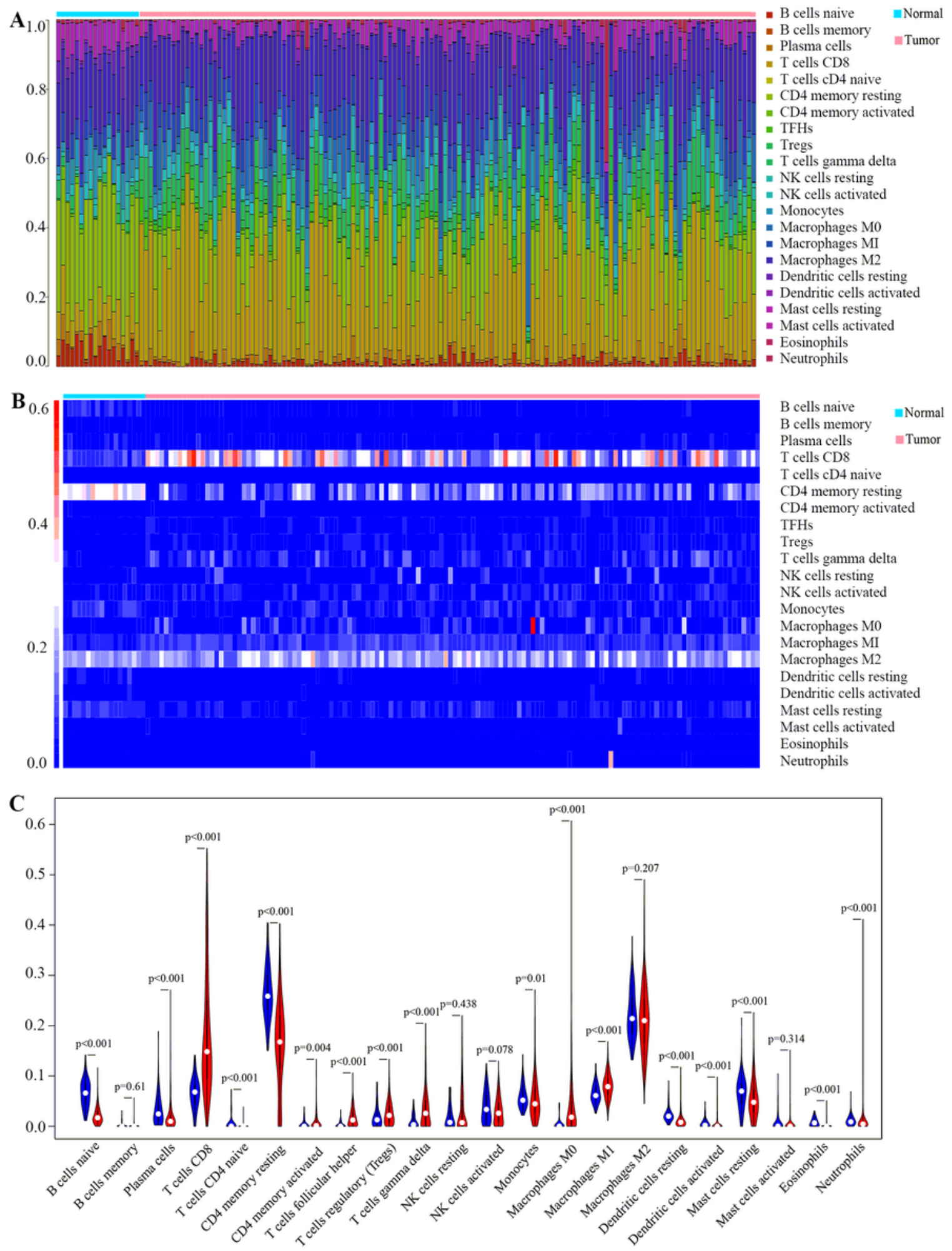

Figure 2

Establishment immune-infiltration landscape A The abundance ratio of immune cells in the samples form TCGA. Each column represents a sample, and each column with a different color and height indicates the abundance ratios of immune cells in this sample. B The heatmap of 22 immune cells in tumor and normal tissues. Each row represents a kind of immune cell and the color of each small square represents 
the content of immune cells. C The difference of 22 immune cell abundance ratios between the ccRCC and control groups in TCGA.
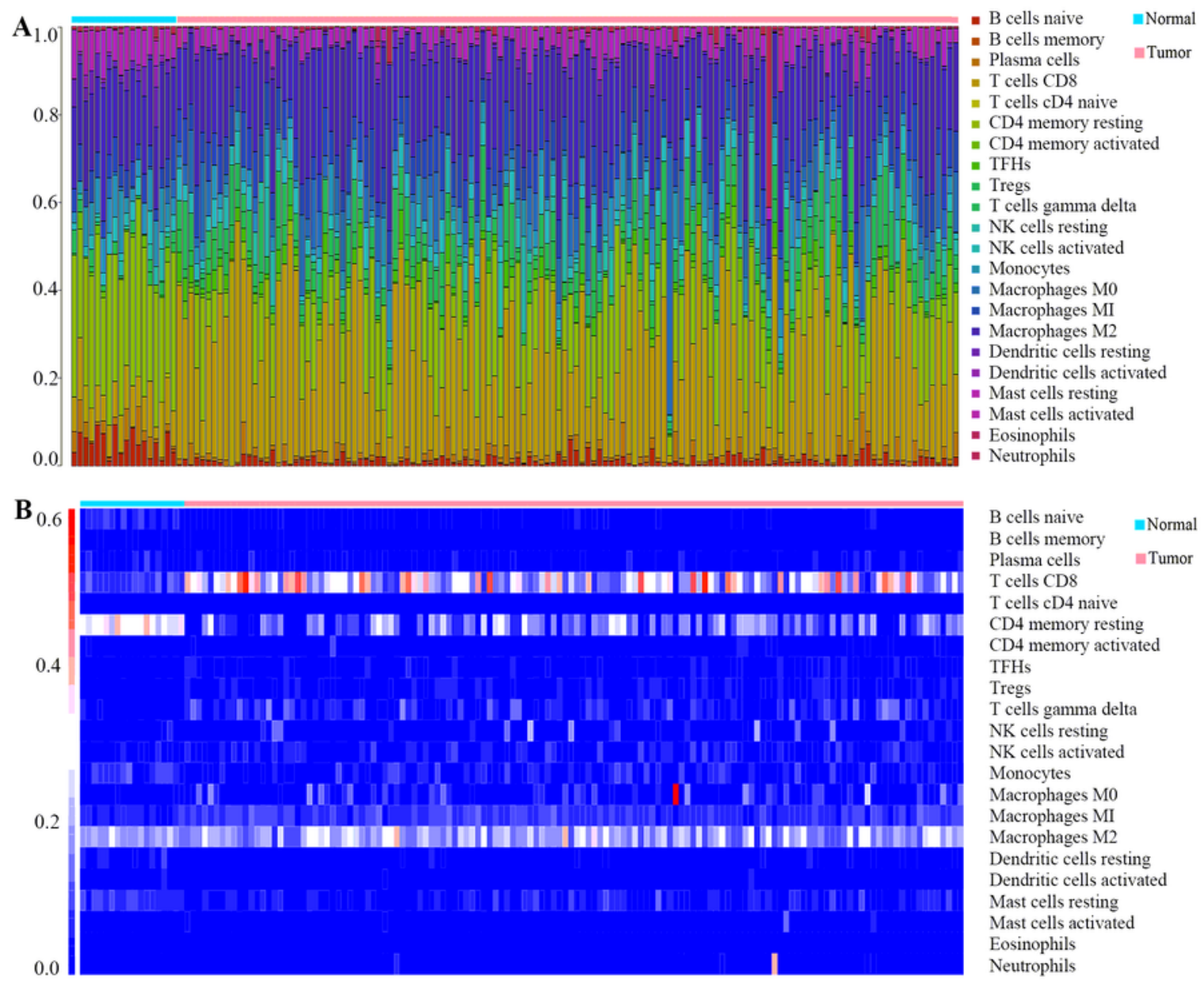
$B$ cells naive B cells memory
Plasma cells $\quad$ Tumor
T cells CD8
T cells $\mathrm{cD} 4$ naive
CD4 memory resting
CD4 memory activated
TFHs
Tregs
$T$ cells gamma delta
NK cells resting
NK cells activated
Monocytes
Macrophages M0
Macrophages MI
Macrophages M2
Dendritic cells resting
Dendritic cells activated
Mast cells resting
Mast cells activated
Eosinophils
Neutrophils

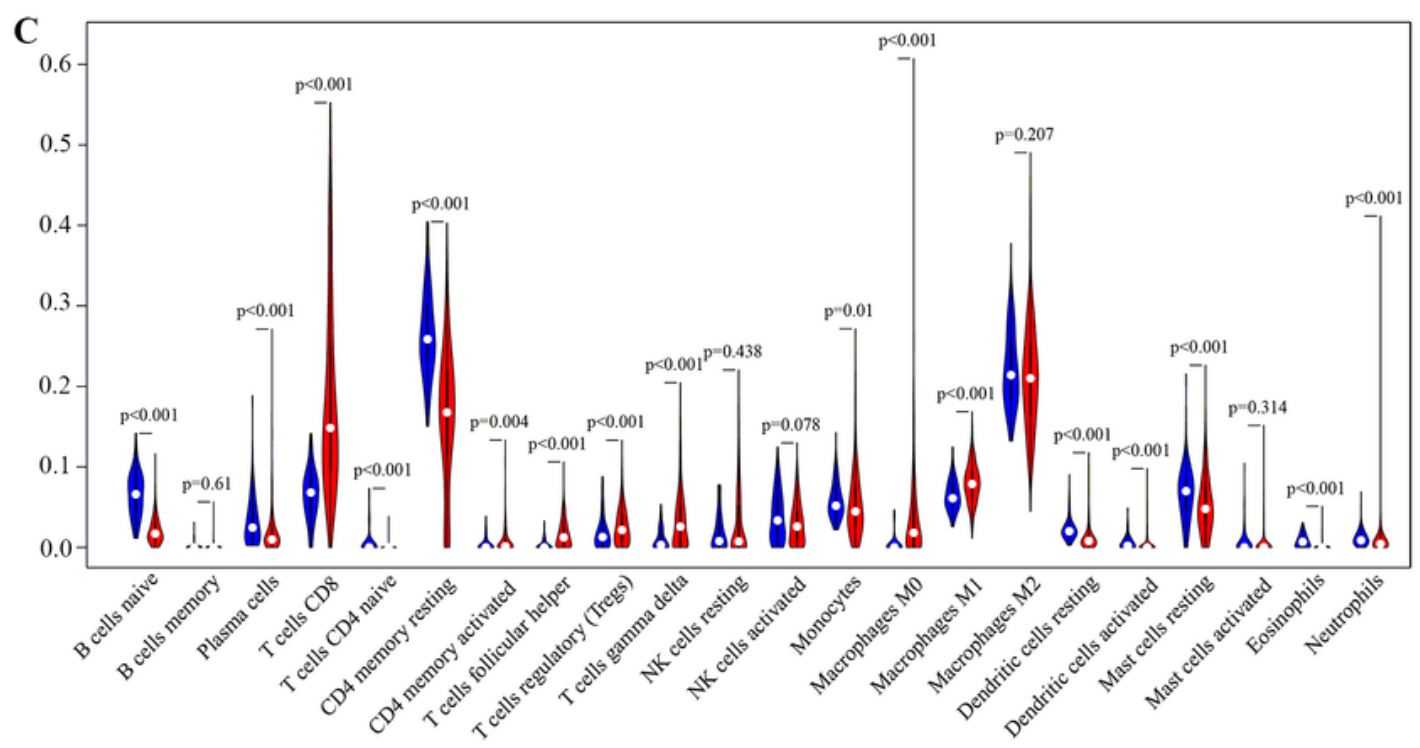

Figure 2

Establishment immune-infiltration landscape A The abundance ratio of immune cells in the samples form TCGA. Each column represents a sample, and each column with a different color and height indicates the abundance ratios of immune cells in this sample. B The heatmap of 22 immune cells in tumor and 
normal tissues. Each row represents a kind of immune cell and the color of each small square represents the content of immune cells. C The difference of 22 immune cell abundance ratios between the ccRCC and control groups in TCGA.
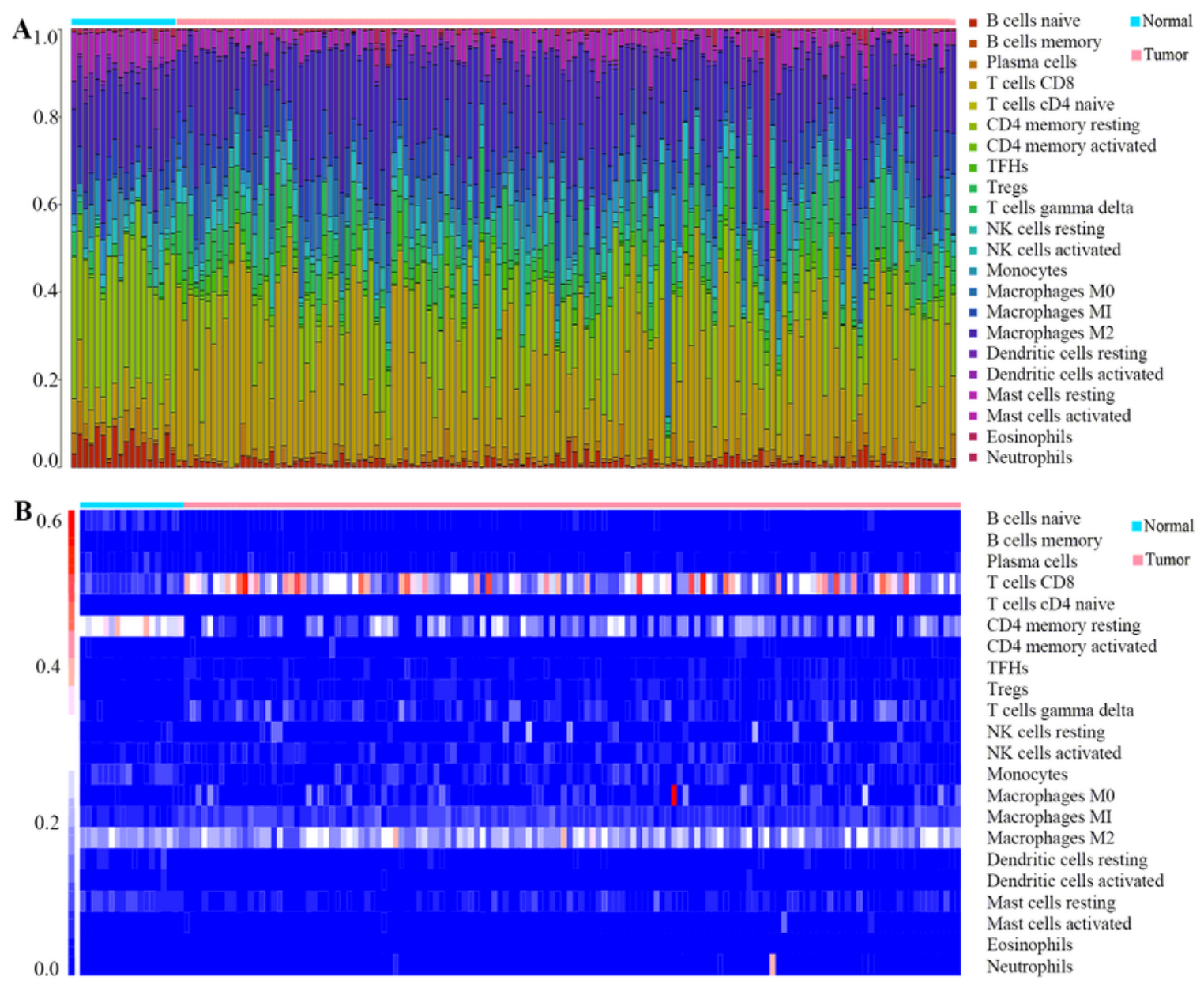

B cells naive
B cells memory
Plasma cells
T cells CD8
T cells cD4 naive
CD4 memory resting
CD4 memory activated
TFHs
Tregs
T cells gamma delta
NK cells resting
NK cells activated
Monocytes
Macrophages M0
Macrophages MI
Macrophages M2
Dendritic cells resting
Dendritic cells activated
Mast cells resting
Mast cells activated
Eosinophils
Neutrophils

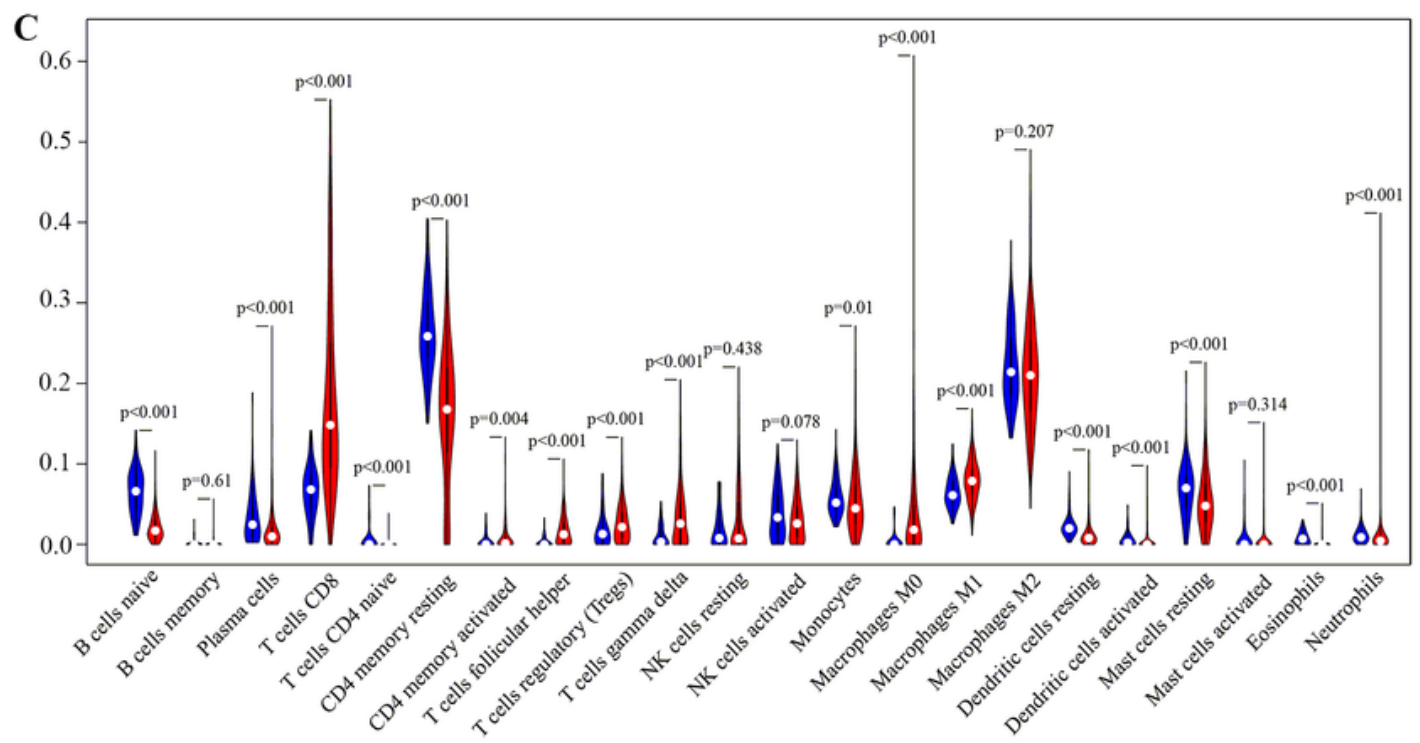

Figure 2

Establishment immune-infiltration landscape A The abundance ratio of immune cells in the samples form TCGA. Each column represents a sample, and each column with a different color and height indicates the 
abundance ratios of immune cells in this sample. B The heatmap of 22 immune cells in tumor and normal tissues. Each row represents a kind of immune cell and the color of each small square represents the content of immune cells. C The difference of 22 immune cell abundance ratios between the ccRCC and control groups in TCGA.
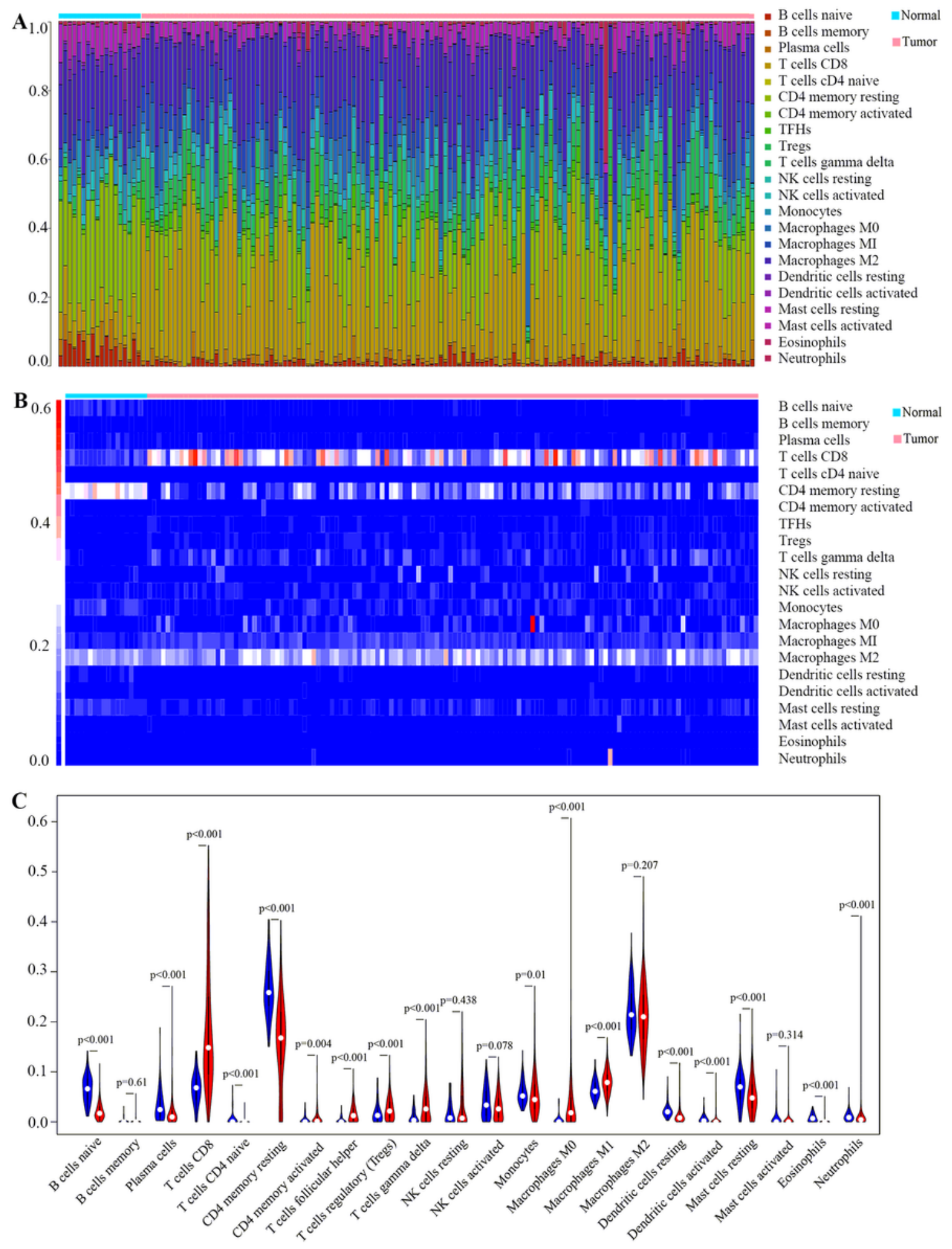

Figure 2 
Establishment immune-infiltration landscape A The abundance ratio of immune cells in the samples form TCGA. Each column represents a sample, and each column with a different color and height indicates the abundance ratios of immune cells in this sample. B The heatmap of 22 immune cells in tumor and normal tissues. Each row represents a kind of immune cell and the color of each small square represents the content of immune cells. C The difference of 22 immune cell abundance ratios between the ccRCC and control groups in TCGA.
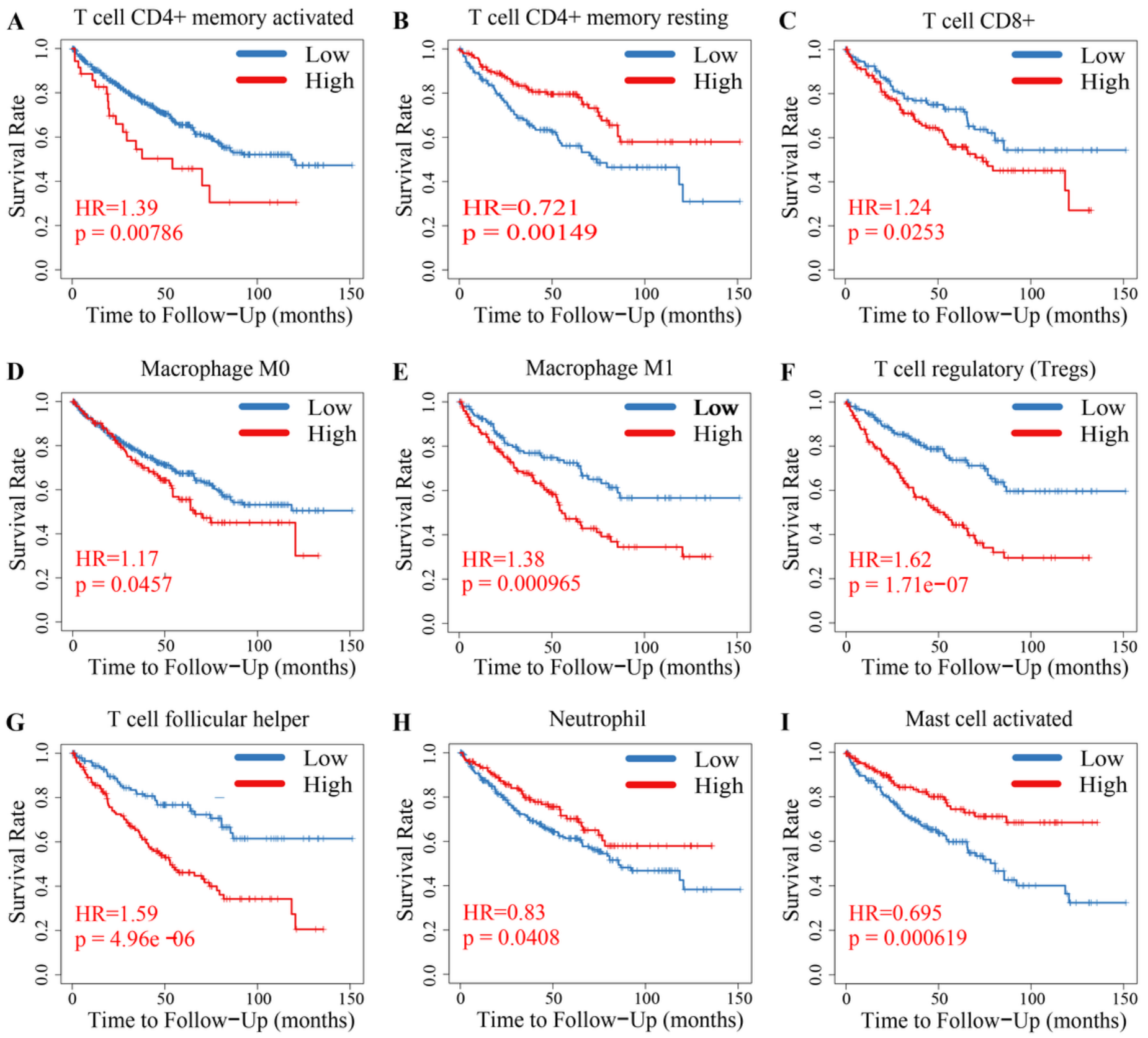

\section{Figure 3}

Identifying Survival-associated Immune Cells A-I The univariate survival analysis for the abundance ratios of the four immune cells. The red line indicates a high expressing group of immune cells, and the blue line indicates a low expressing group of immune cells. 
A T cell CD4+ memory activated

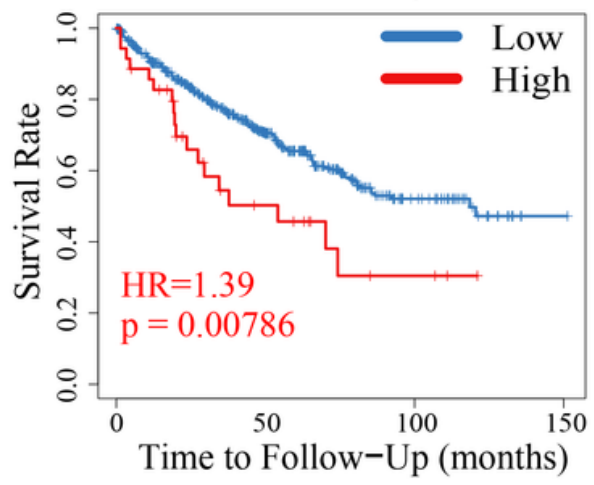

D

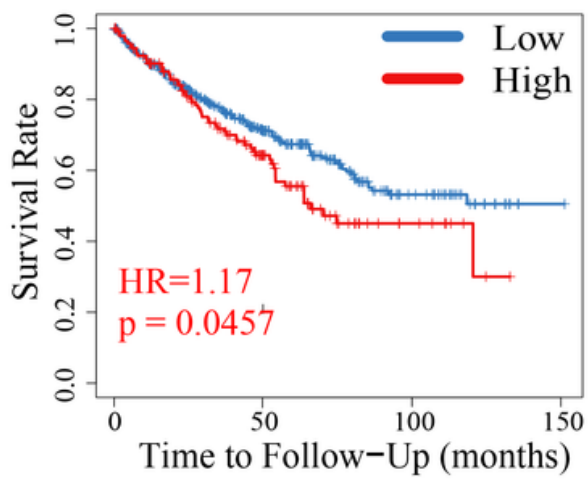

G T cell follicular helper

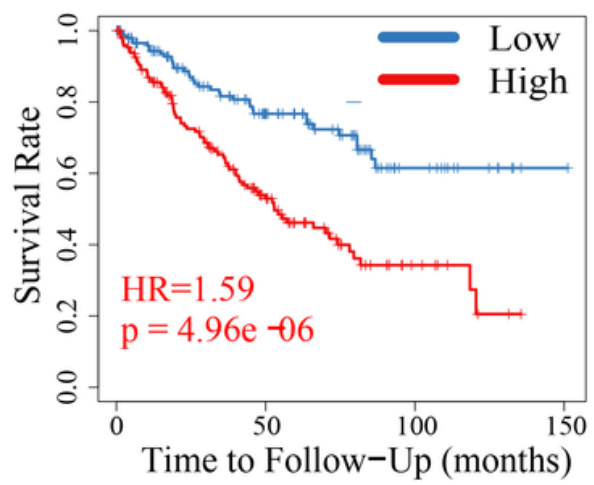

B T cell CD4+ memory resting

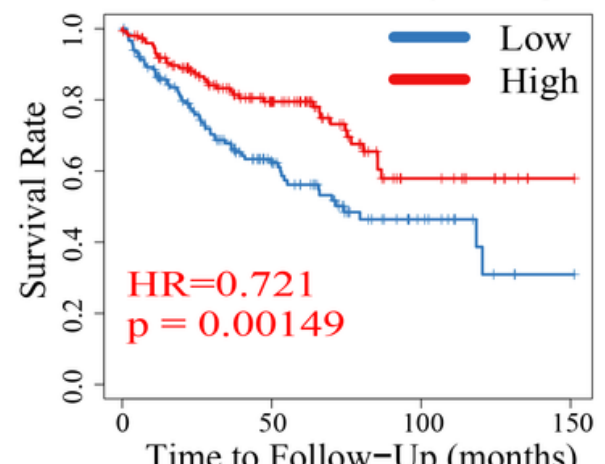

Time to Follow-Up (months)

E

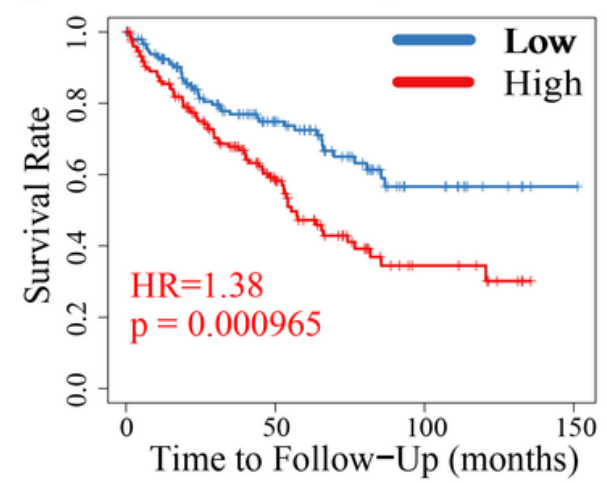

H

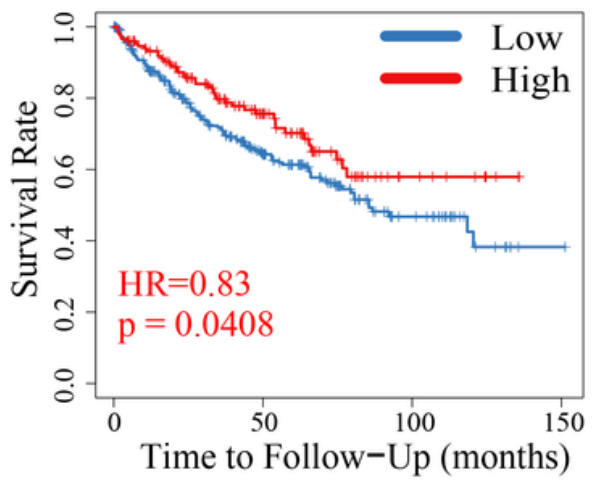

C T cell CD8+

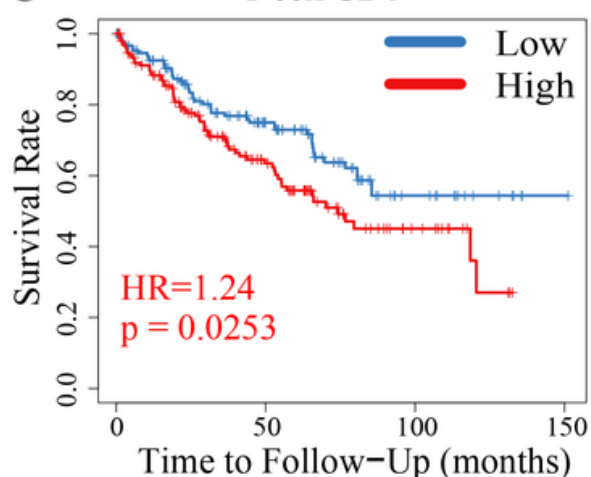

F T cell regulatory (Tregs)

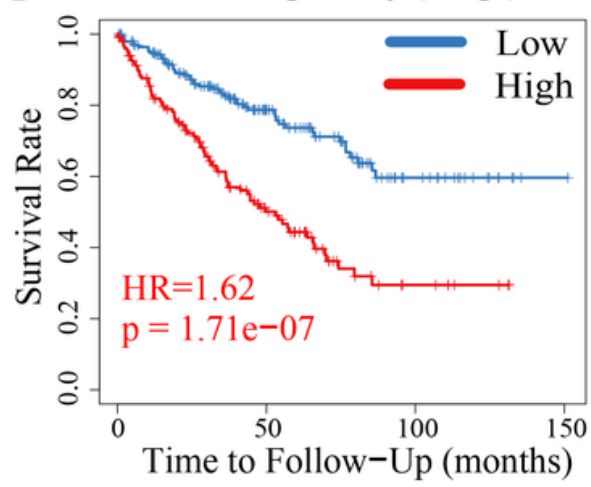

I Mast cell activated

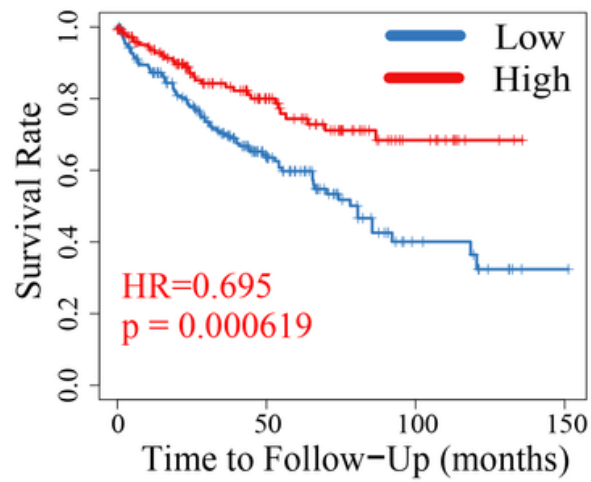

\section{Figure 3}

Identifying Survival-associated Immune Cells A-I The univariate survival analysis for the abundance ratios of the four immune cells. The red line indicates a high expressing group of immune cells, and the blue line indicates a low expressing group of immune cells. 
A T cell CD4+ memory activated

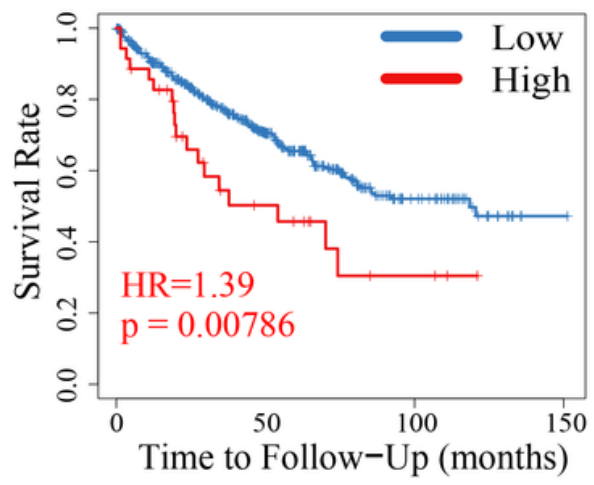

D

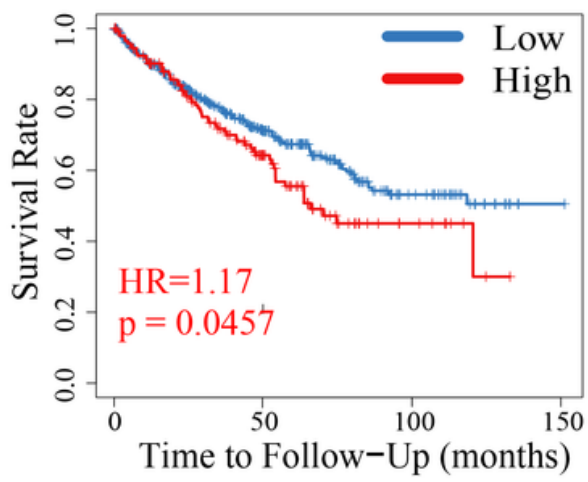

G T cell follicular helper

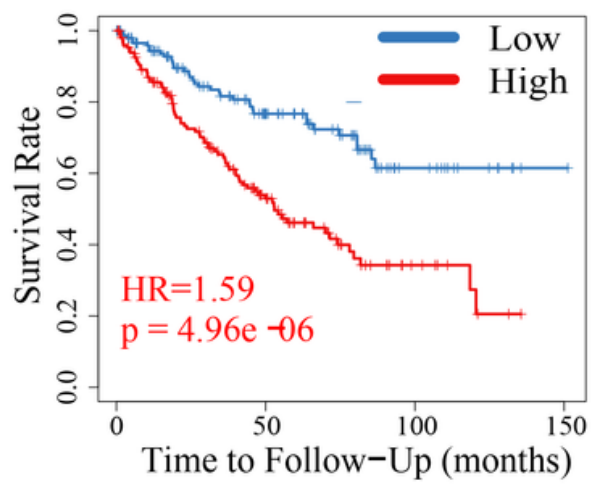

B T cell CD4+ memory resting

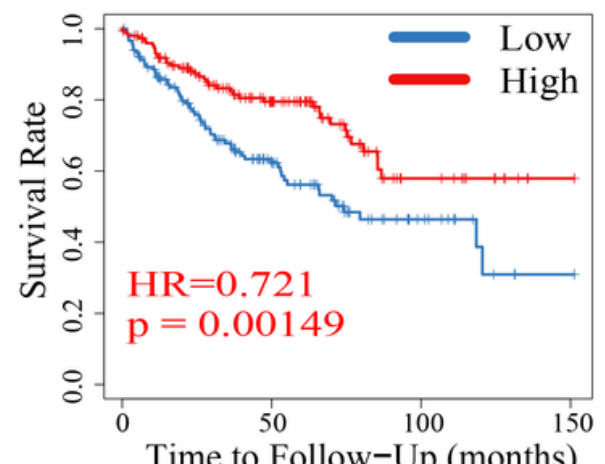

Time to Follow-Up (months)

E

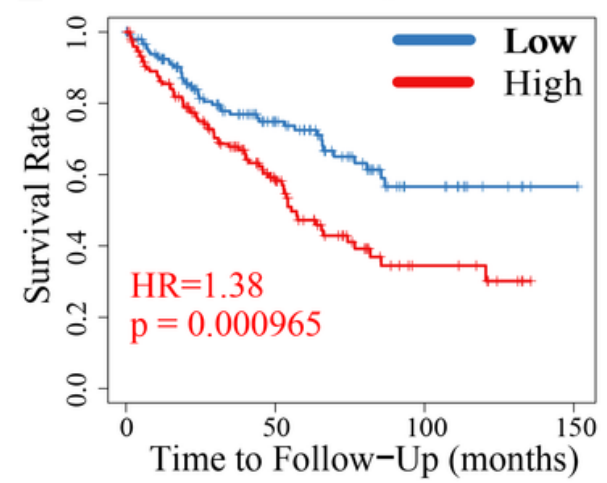

H

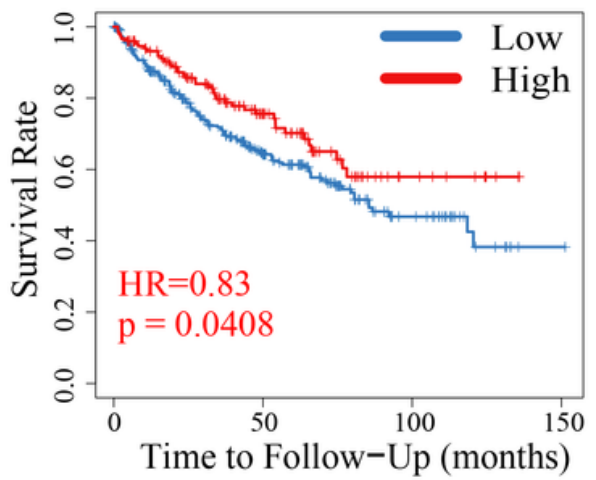

C T cell CD8+

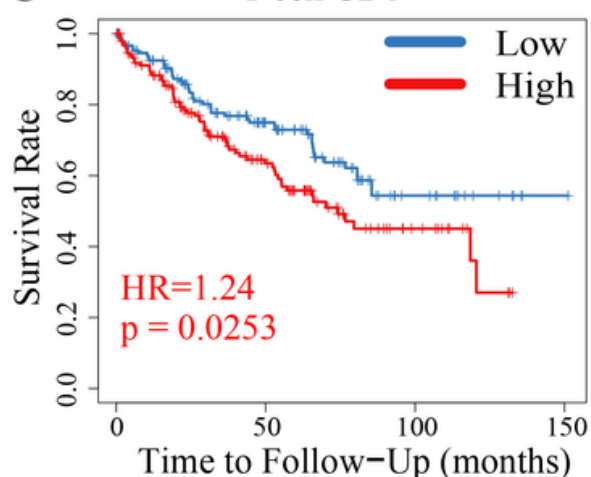

F T cell regulatory (Tregs)

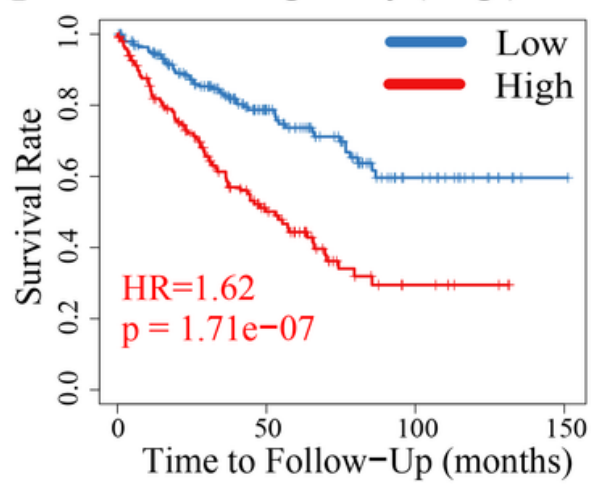

I Mast cell activated

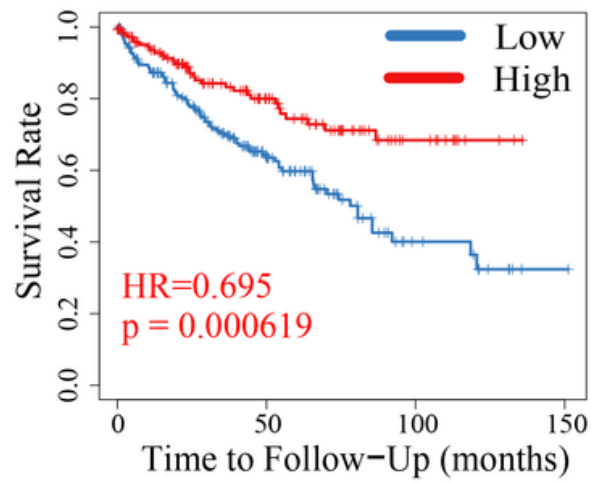

\section{Figure 3}

Identifying Survival-associated Immune Cells A-I The univariate survival analysis for the abundance ratios of the four immune cells. The red line indicates a high expressing group of immune cells, and the blue line indicates a low expressing group of immune cells. 
A T cell CD4+ memory activated

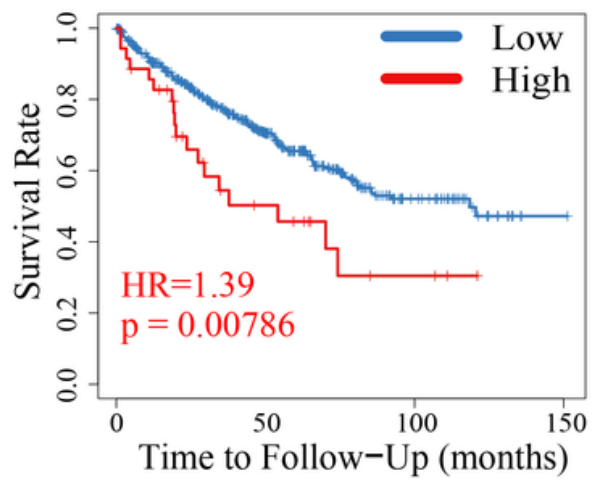

D

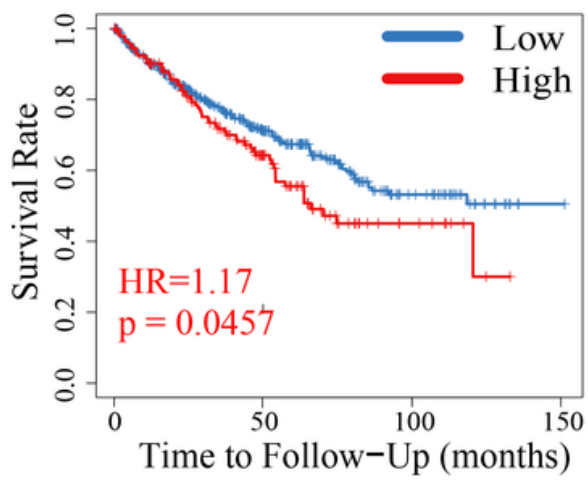

G T cell follicular helper

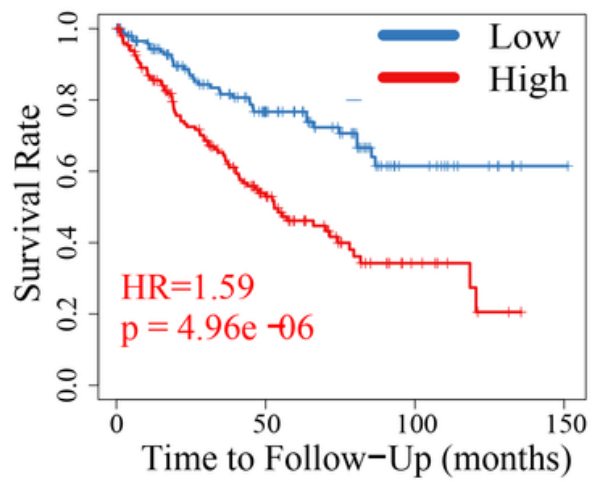

B T cell CD4+ memory resting

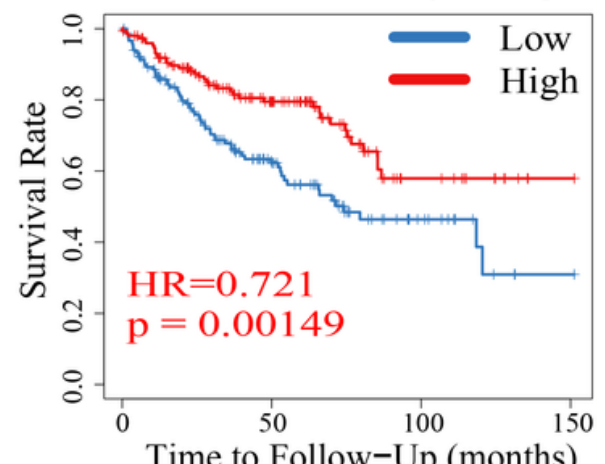

Time to Follow-Up (months)

E

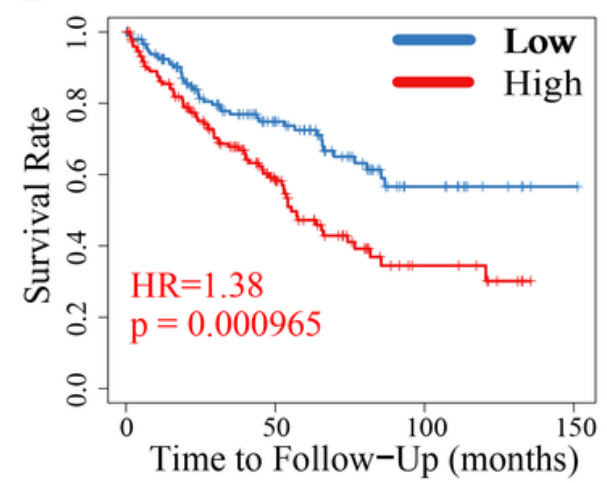

H

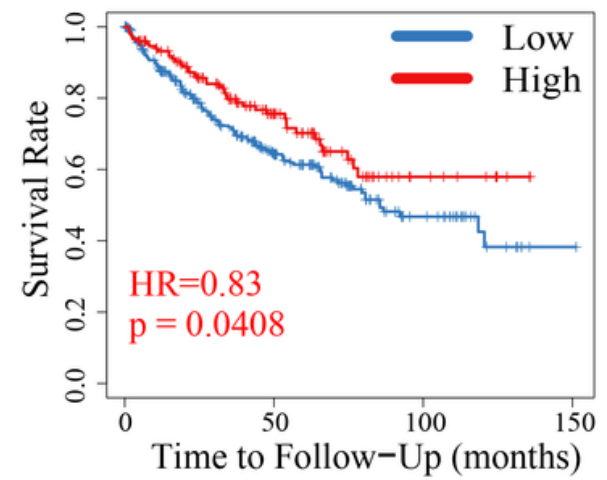

C T cell CD8+

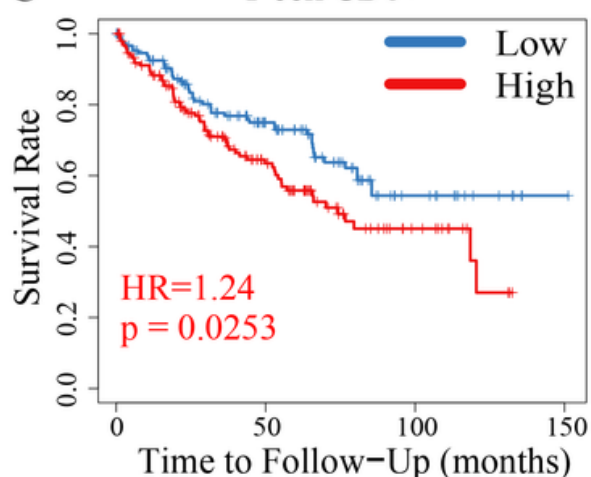

F T cell regulatory (Tregs)

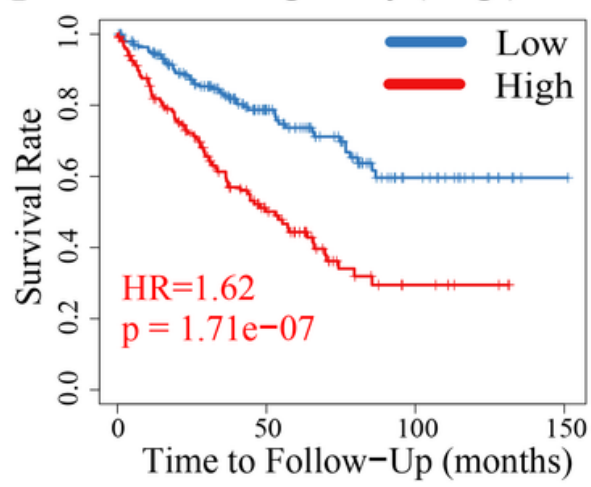

I Mast cell activated

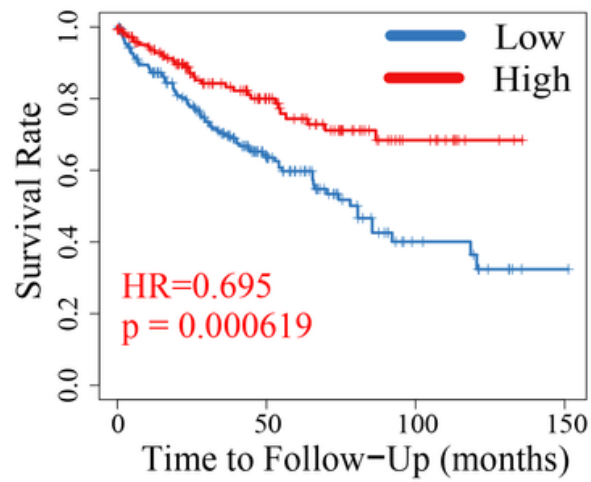

\section{Figure 3}

Identifying Survival-associated Immune Cells A-I The univariate survival analysis for the abundance ratios of the four immune cells. The red line indicates a high expressing group of immune cells, and the blue line indicates a low expressing group of immune cells. 

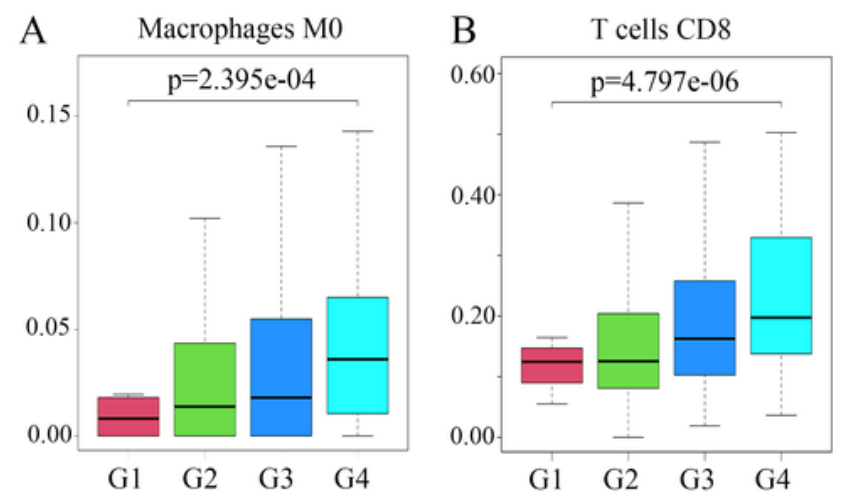

C T cells folicular helper

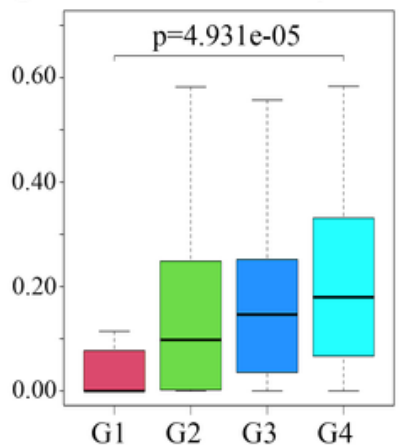

E T cells CD4 memory resting

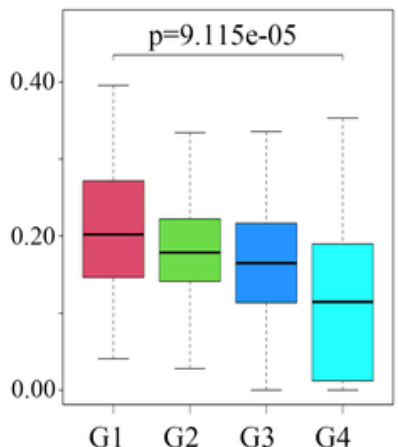

F T cells CD4 memory activated
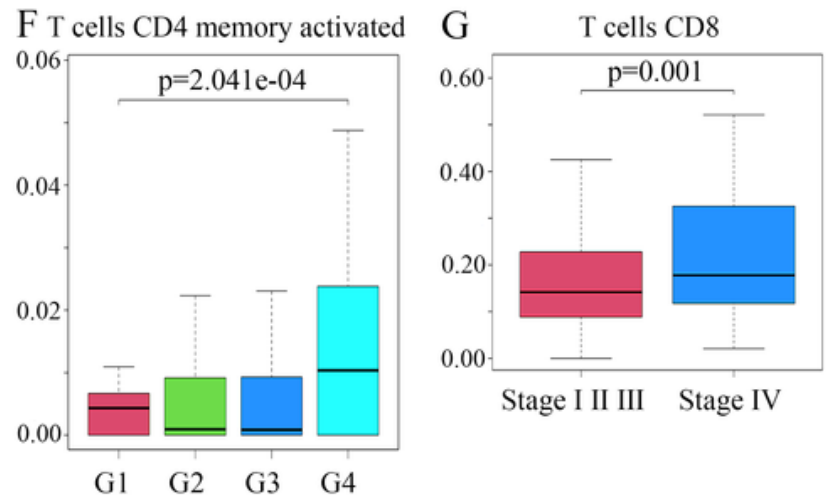

Stage I II III Stage IV

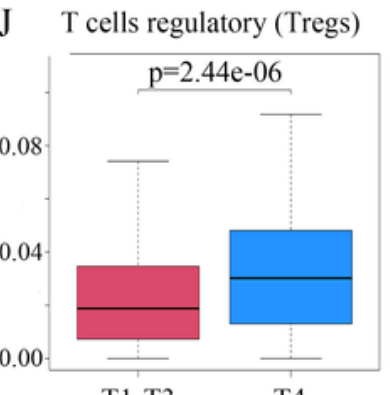

I T cells regulatory (Tregs)

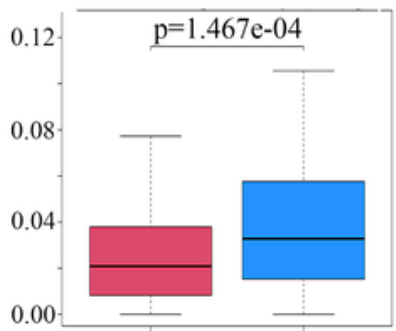

Stage I II III Stage IV

M T cells regulatory (Tregs)

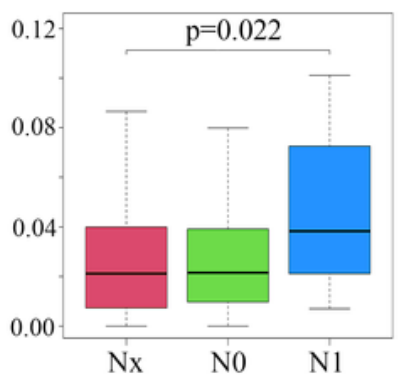

$\mathrm{N}$ T cells regulatory (Tregs)

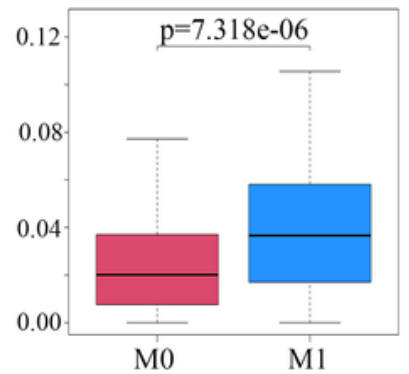

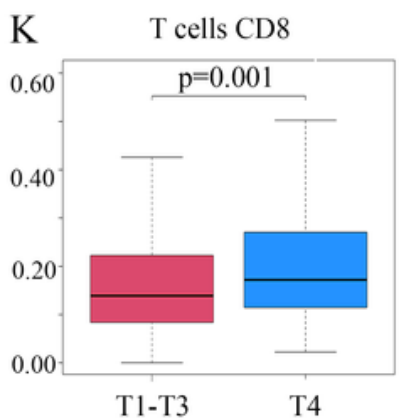

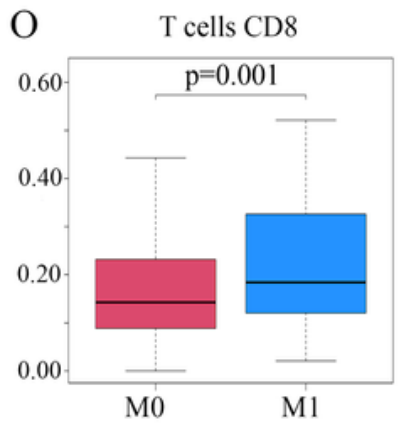

D T cells regulatory (Tregs)

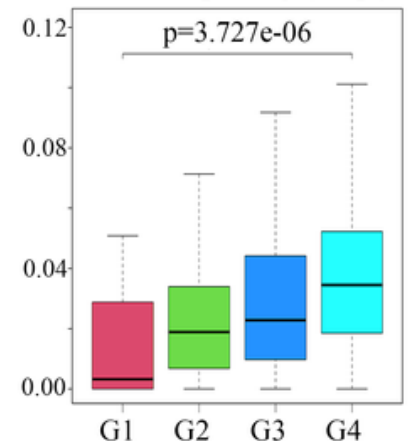

$\mathrm{H}$ T cells folicular helper

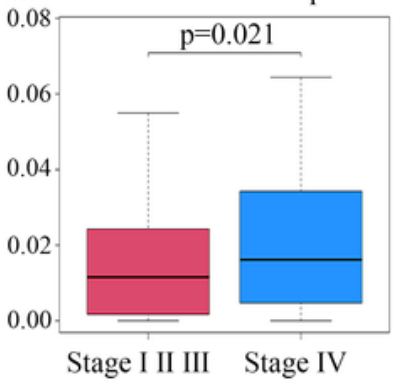

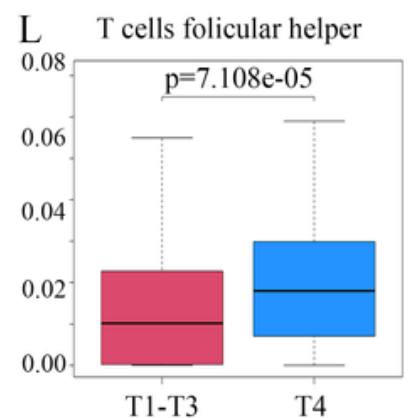

\section{Figure 4}

Relationship between clinical attributes and survival-associated immune cells A-J The relationship between the abundance ratios of the immune cells and clinical stage. G-P The relationship between the abundance ratios of each immune cell and clinical stage, $\mathrm{T}$ stage, $\mathrm{M}$ stage and $\mathrm{N}$ stage. The ordinate represents the content of immune cells and the horizontal line inside the box represents the median value of immune cell contents. 

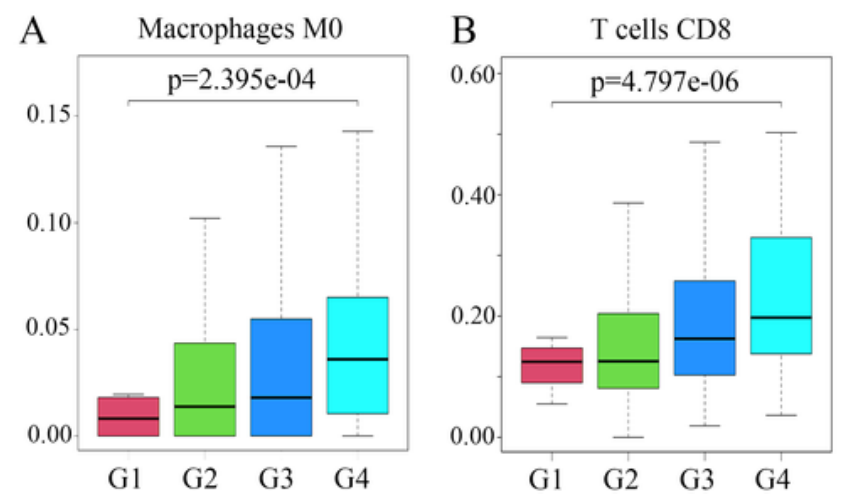

C T cells folicular helper

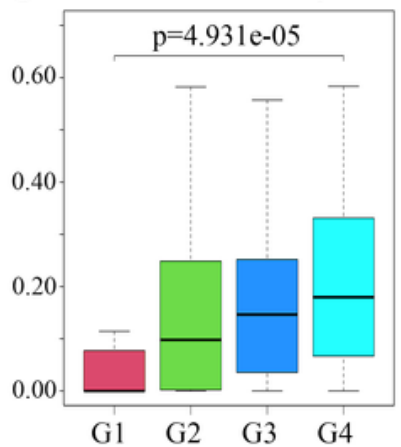

E T cells CD4 memory resting

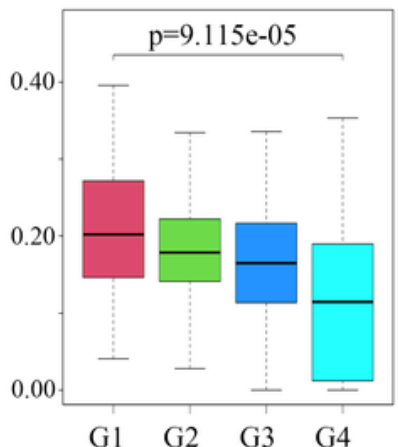

F T cells CD4 memory activated
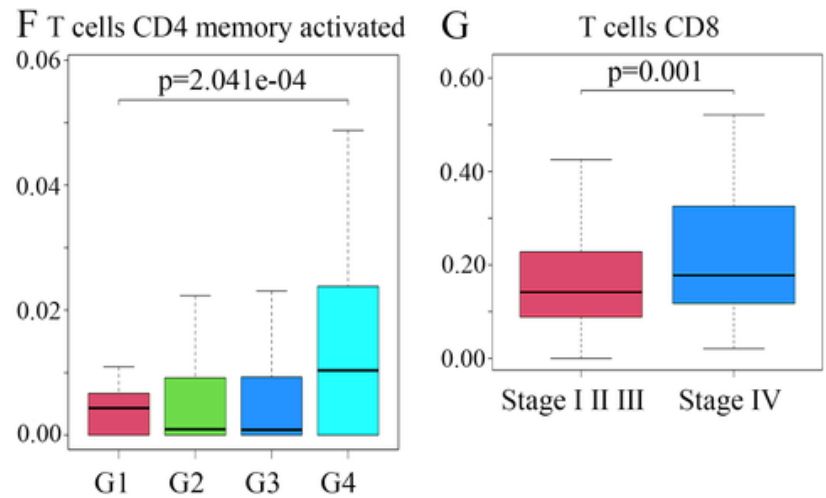

Stage I II III Stage IV

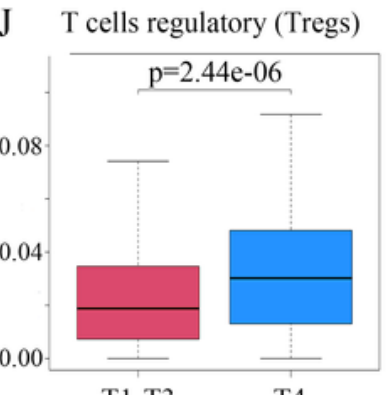

I T cells regulatory (Tregs)

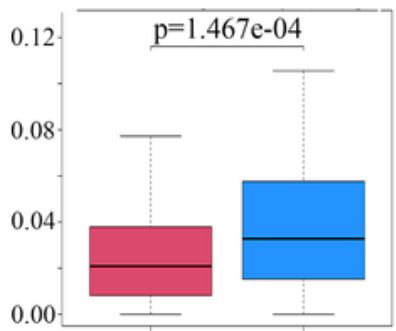

Stage I II III Stage IV

M T cells regulatory (Tregs)

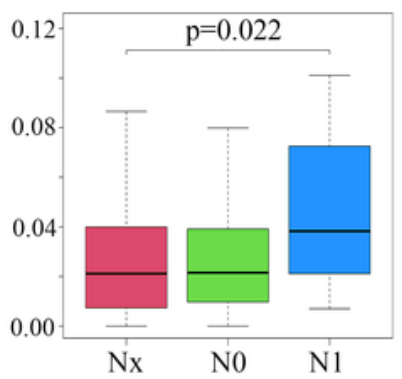

$\mathrm{N}$ T cells regulatory (Tregs)

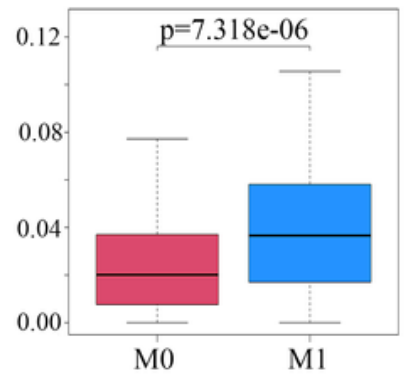

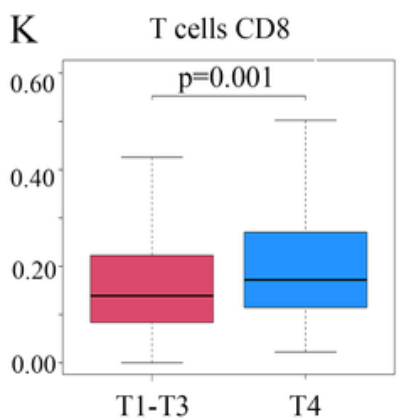

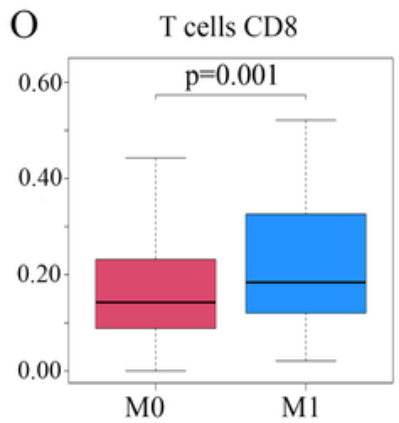

D T cells regulatory (Tregs)

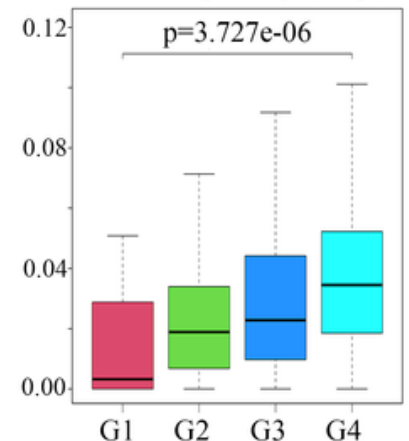

$\mathrm{H}$ T cells folicular helper

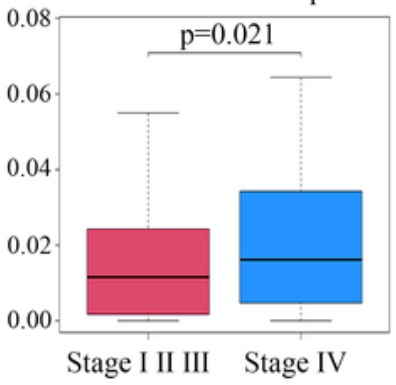

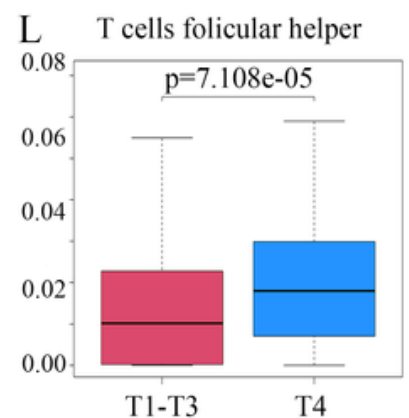

\section{Figure 4}

Relationship between clinical attributes and survival-associated immune cells A-J The relationship between the abundance ratios of the immune cells and clinical stage. G-P The relationship between the abundance ratios of each immune cell and clinical stage, $\mathrm{T}$ stage, $\mathrm{M}$ stage and $\mathrm{N}$ stage. The ordinate represents the content of immune cells and the horizontal line inside the box represents the median value of immune cell contents. 

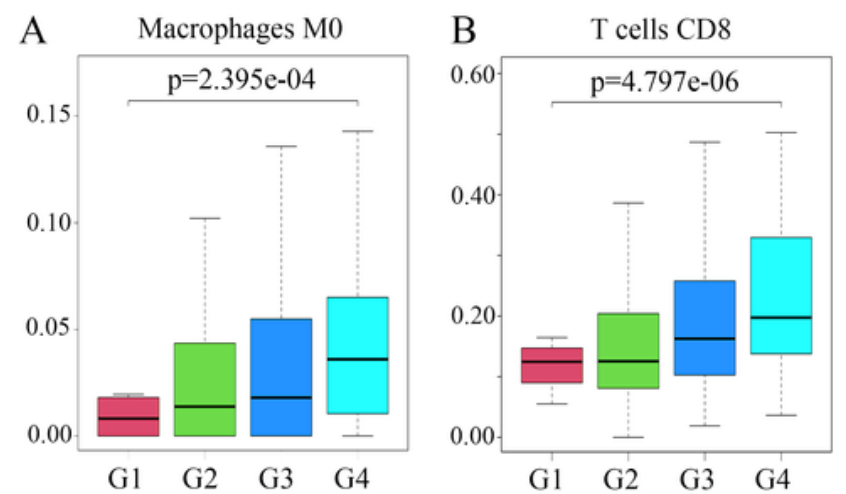

C T cells folicular helper

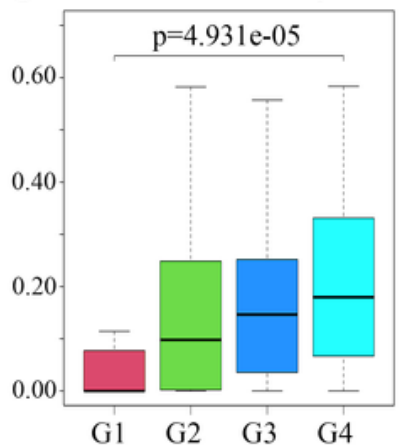

E T cells CD4 memory resting

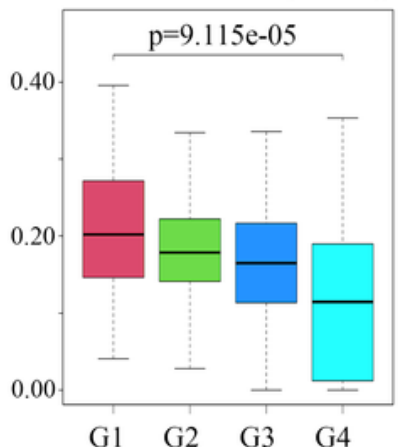

F T cells CD4 memory activated
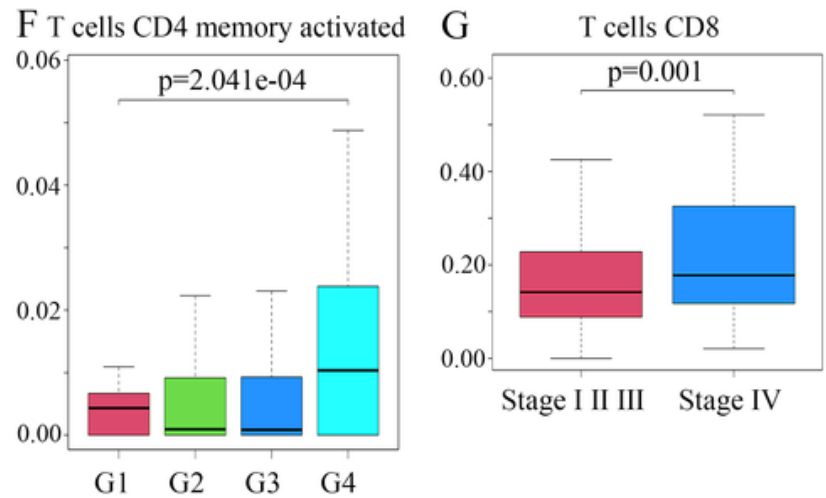

Stage I II III Stage IV

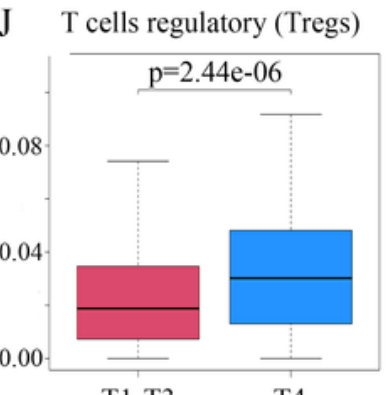

I T cells regulatory (Tregs)

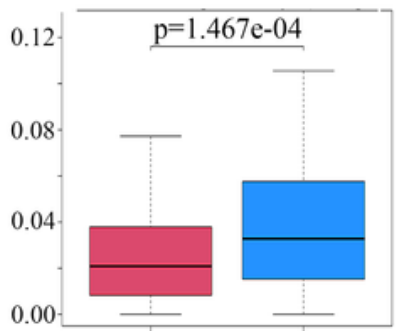

Stage I II III Stage IV

M T cells regulatory (Tregs)

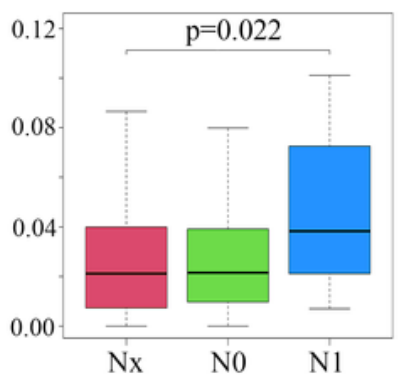

$\mathrm{N}$ T cells regulatory (Tregs)

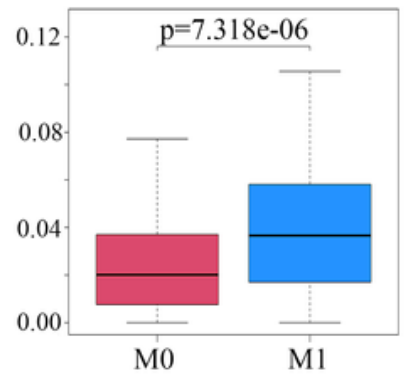

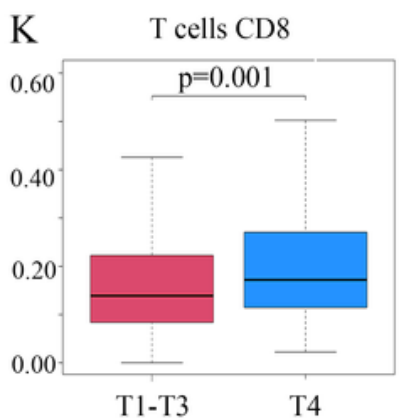

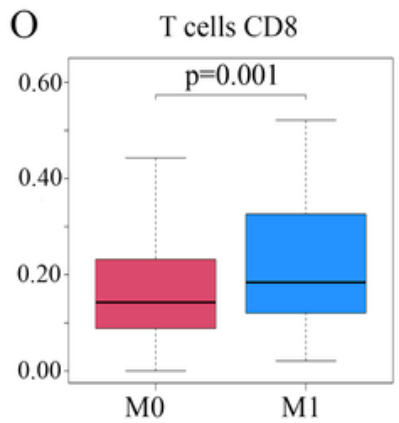

D T cells regulatory (Tregs)

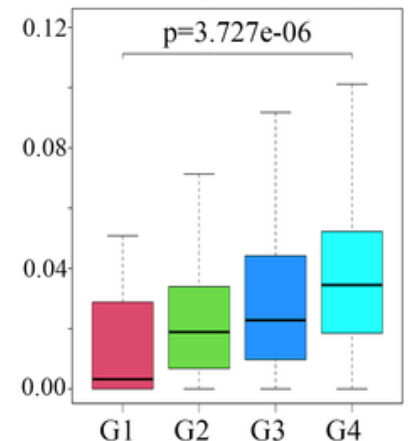

$\mathrm{H}$ T cells folicular helper

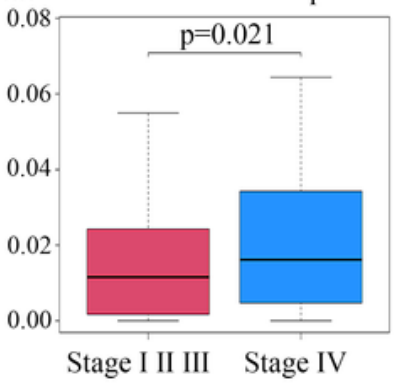

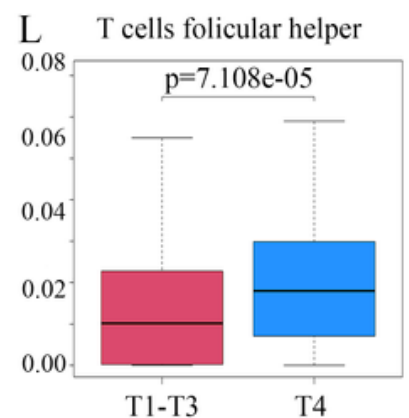

\section{Figure 4}

Relationship between clinical attributes and survival-associated immune cells A-J The relationship between the abundance ratios of the immune cells and clinical stage. G-P The relationship between the abundance ratios of each immune cell and clinical stage, $\mathrm{T}$ stage, $\mathrm{M}$ stage and $\mathrm{N}$ stage. The ordinate represents the content of immune cells and the horizontal line inside the box represents the median value of immune cell contents. 

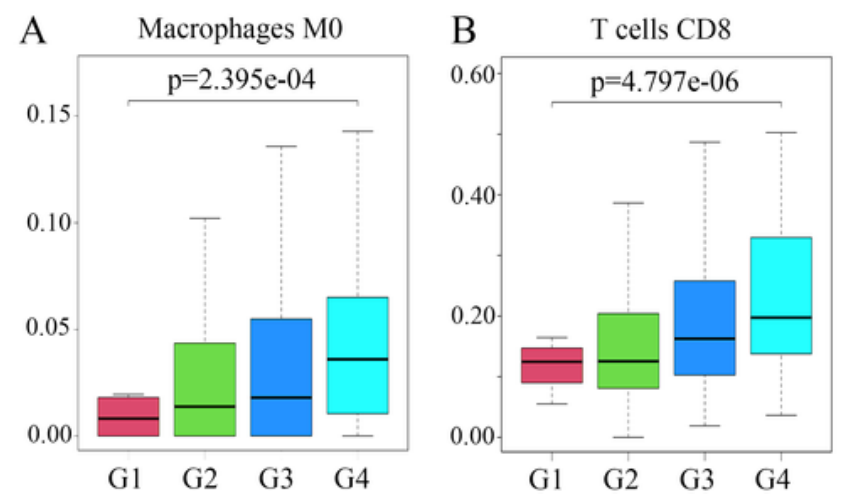

C T cells folicular helper

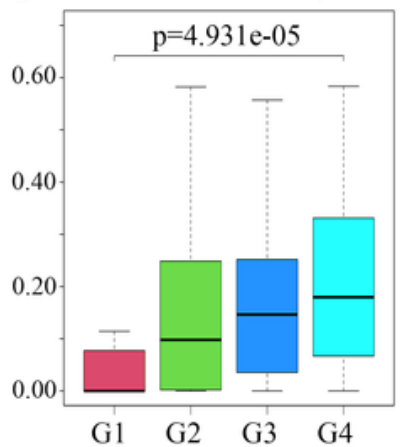

E T cells CD4 memory resting

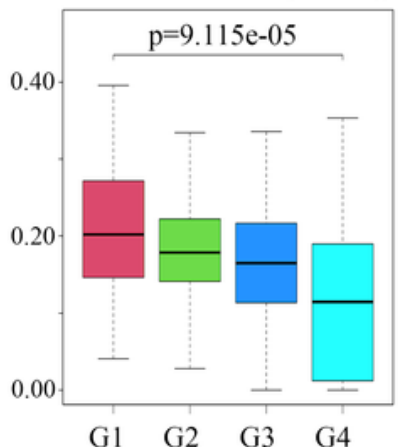

F T cells CD4 memory activated
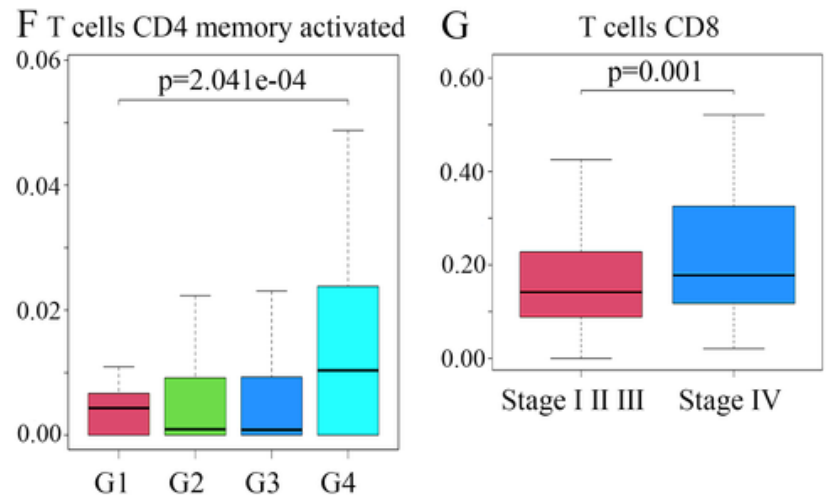

Stage I II III Stage IV

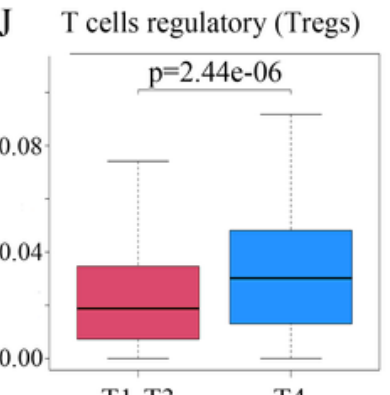

I T cells regulatory (Tregs)

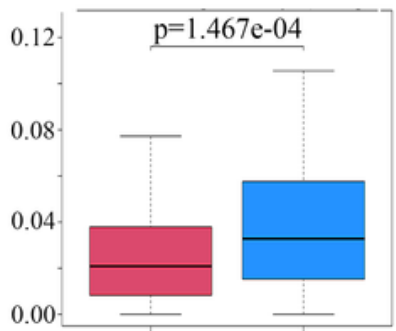

Stage I II III Stage IV

M T cells regulatory (Tregs)

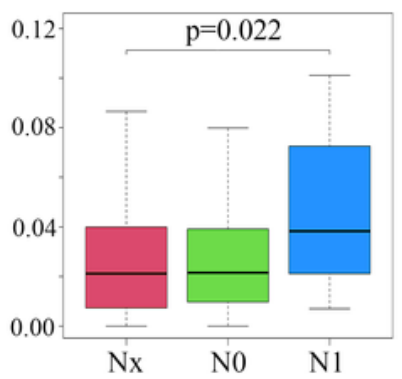

$\mathrm{N}$ T cells regulatory (Tregs)

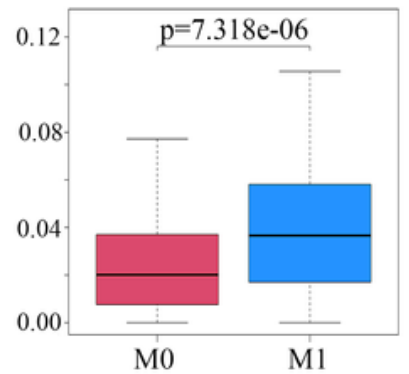

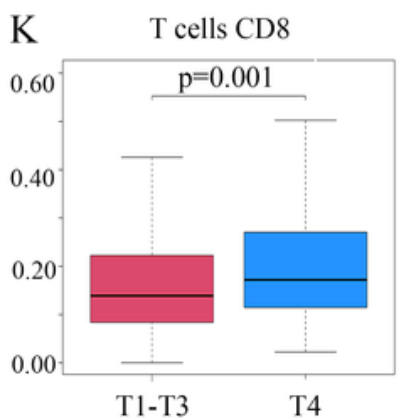

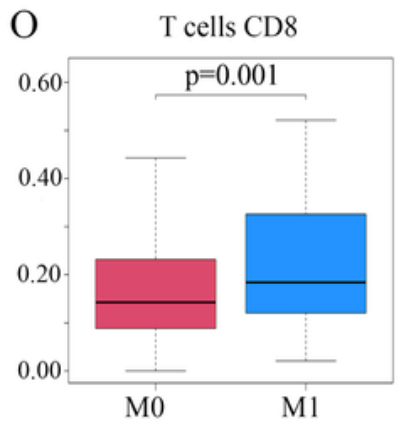

D T cells regulatory (Tregs)

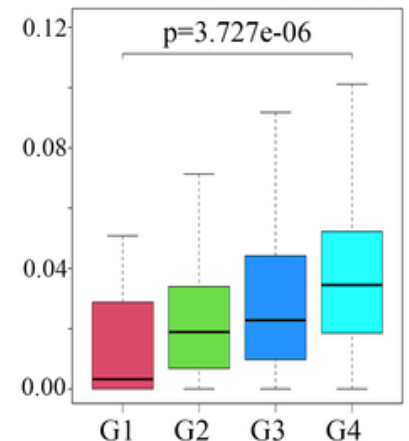

$\mathrm{H}$ T cells folicular helper

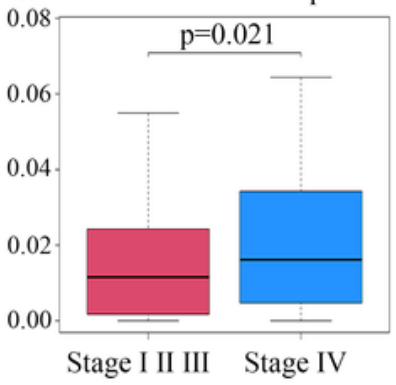

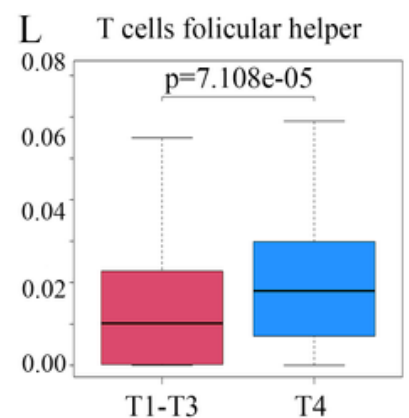

\section{Figure 4}

Relationship between clinical attributes and survival-associated immune cells A-J The relationship between the abundance ratios of the immune cells and clinical stage. G-P The relationship between the abundance ratios of each immune cell and clinical stage, $\mathrm{T}$ stage, $\mathrm{M}$ stage and $\mathrm{N}$ stage. The ordinate represents the content of immune cells and the horizontal line inside the box represents the median value of immune cell contents. 


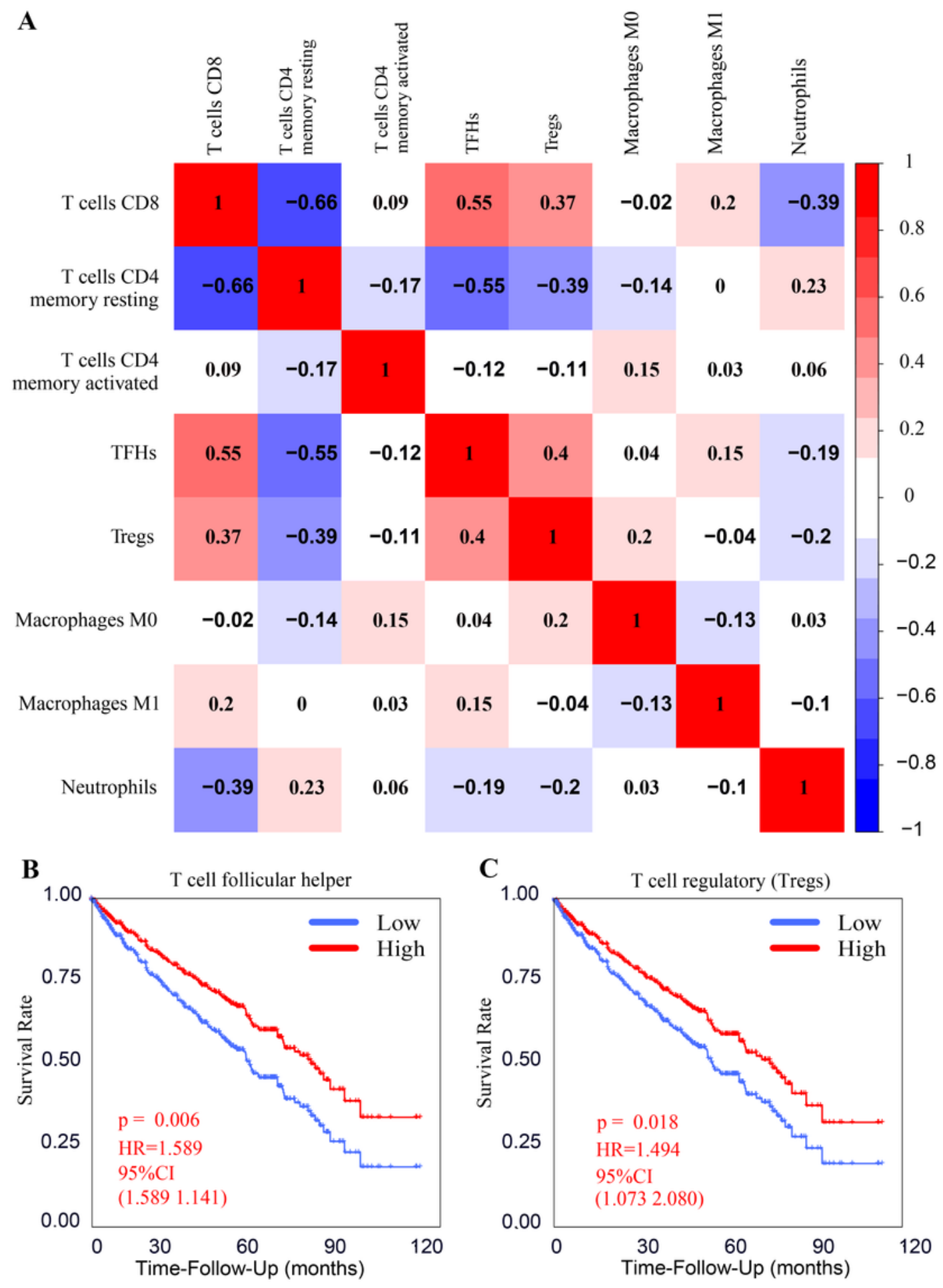

\section{Figure 5}

Screening crucial survival-associated immune cells A Correlation matrix of all 8 immune cell proportions and the color of each small square and the number inside both represent the correlation between the two cells. B The multivariate survival analysis for the abundance ratios of immune cells. The red line indicates a high expressing group of immune cells, and the blue line indicates a low expressing group of immune cells. HR means hazard ratio and $\mathrm{Cl}$ means confidence interval. 


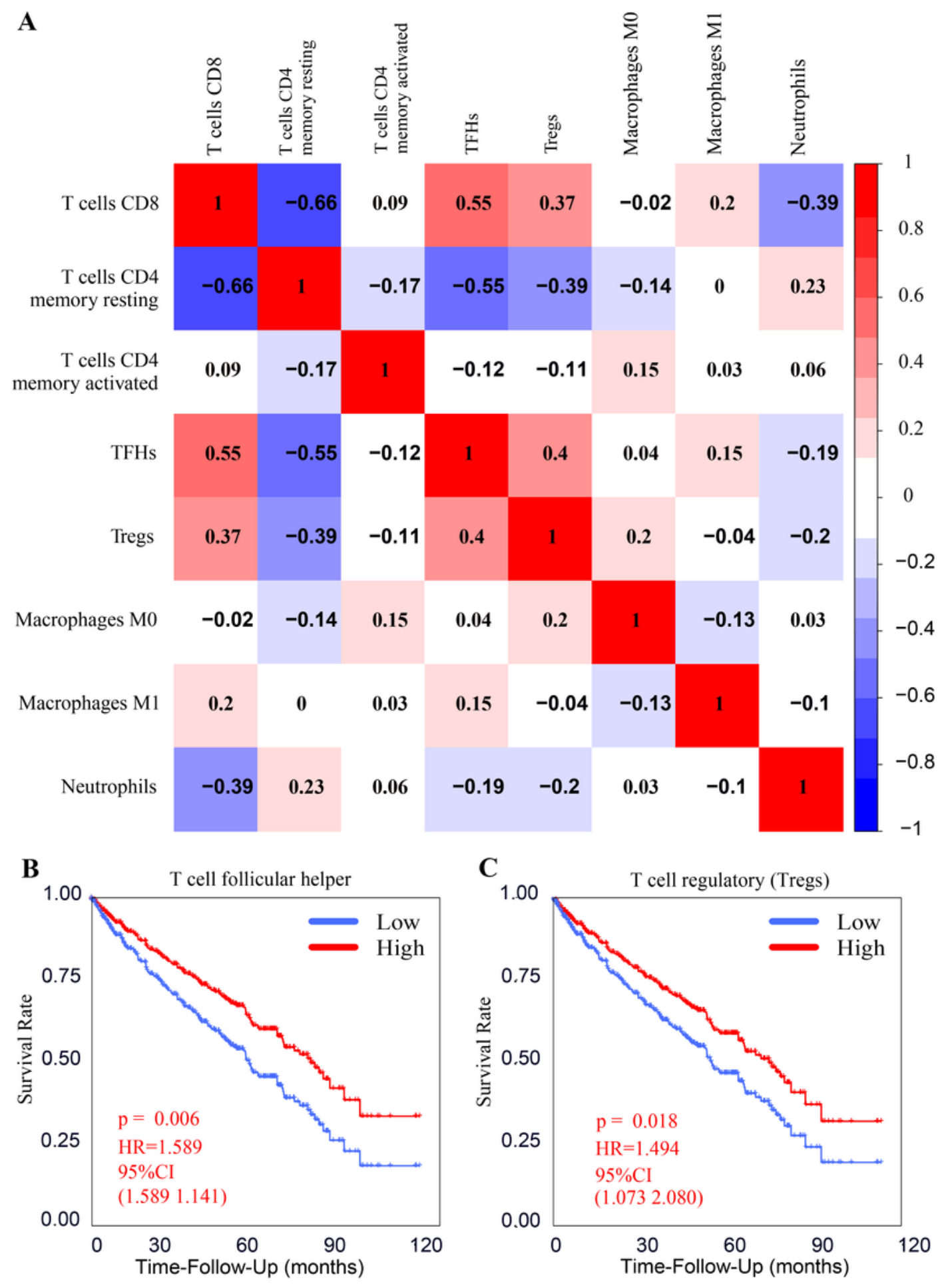

\section{Figure 5}

Screening crucial survival-associated immune cells A Correlation matrix of all 8 immune cell proportions and the color of each small square and the number inside both represent the correlation between the two cells. B The multivariate survival analysis for the abundance ratios of immune cells. The red line indicates a high expressing group of immune cells, and the blue line indicates a low expressing group of immune cells. HR means hazard ratio and $\mathrm{Cl}$ means confidence interval. 


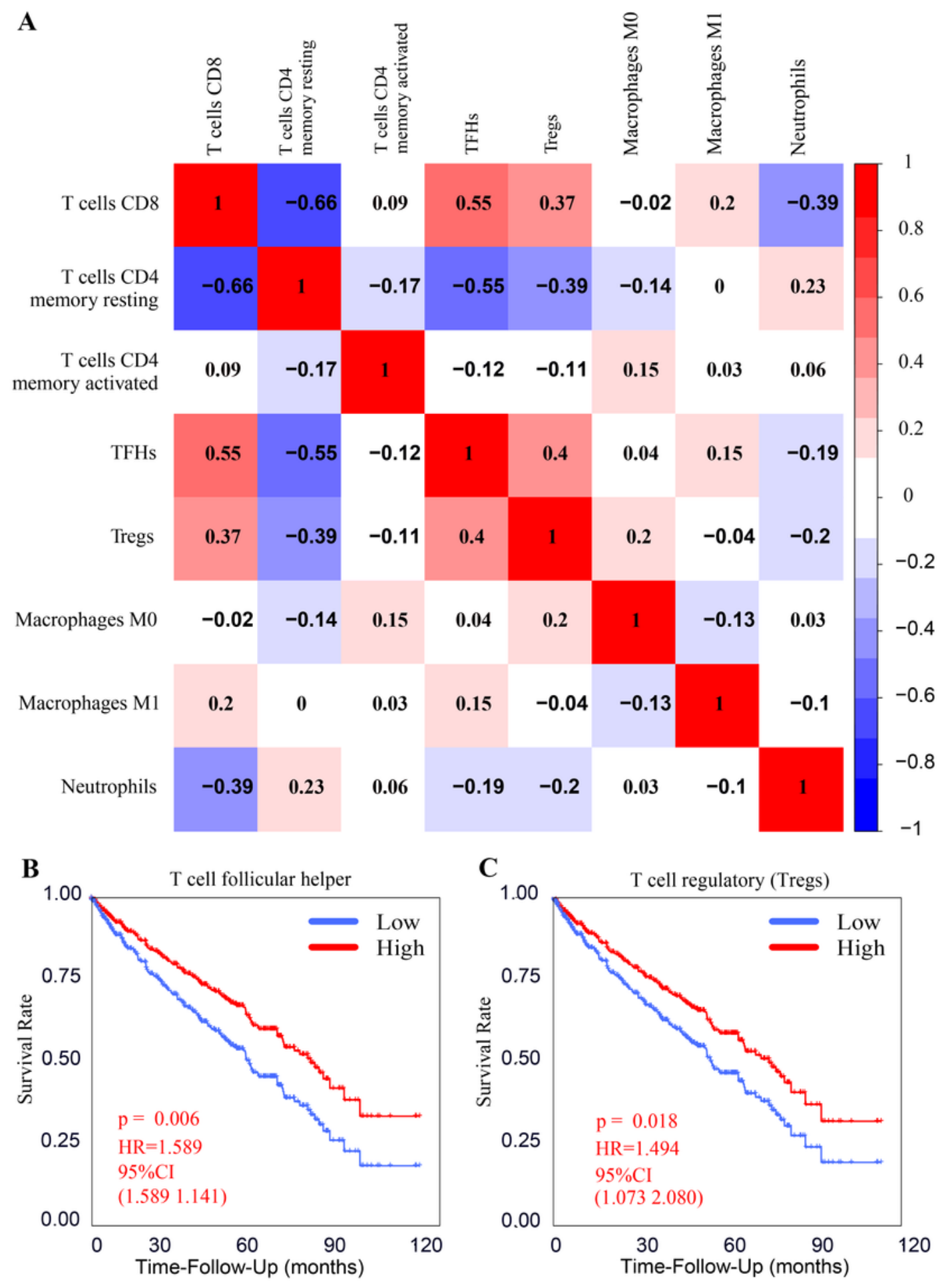

\section{Figure 5}

Screening crucial survival-associated immune cells A Correlation matrix of all 8 immune cell proportions and the color of each small square and the number inside both represent the correlation between the two cells. B The multivariate survival analysis for the abundance ratios of immune cells. The red line indicates a high expressing group of immune cells, and the blue line indicates a low expressing group of immune cells. HR means hazard ratio and $\mathrm{Cl}$ means confidence interval. 


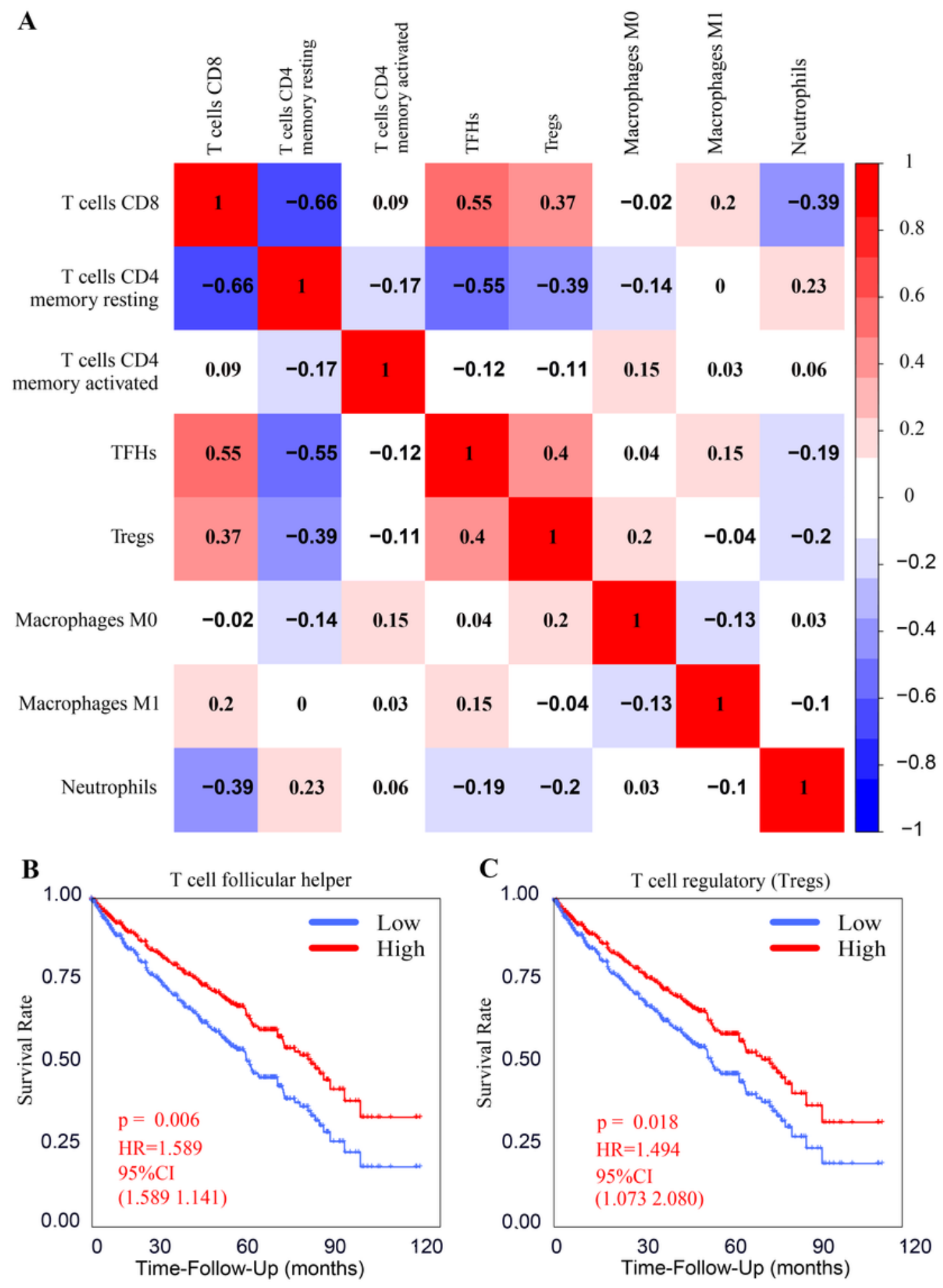

\section{Figure 5}

Screening crucial survival-associated immune cells A Correlation matrix of all 8 immune cell proportions and the color of each small square and the number inside both represent the correlation between the two cells. B The multivariate survival analysis for the abundance ratios of immune cells. The red line indicates a high expressing group of immune cells, and the blue line indicates a low expressing group of immune cells. HR means hazard ratio and $\mathrm{Cl}$ means confidence interval. 

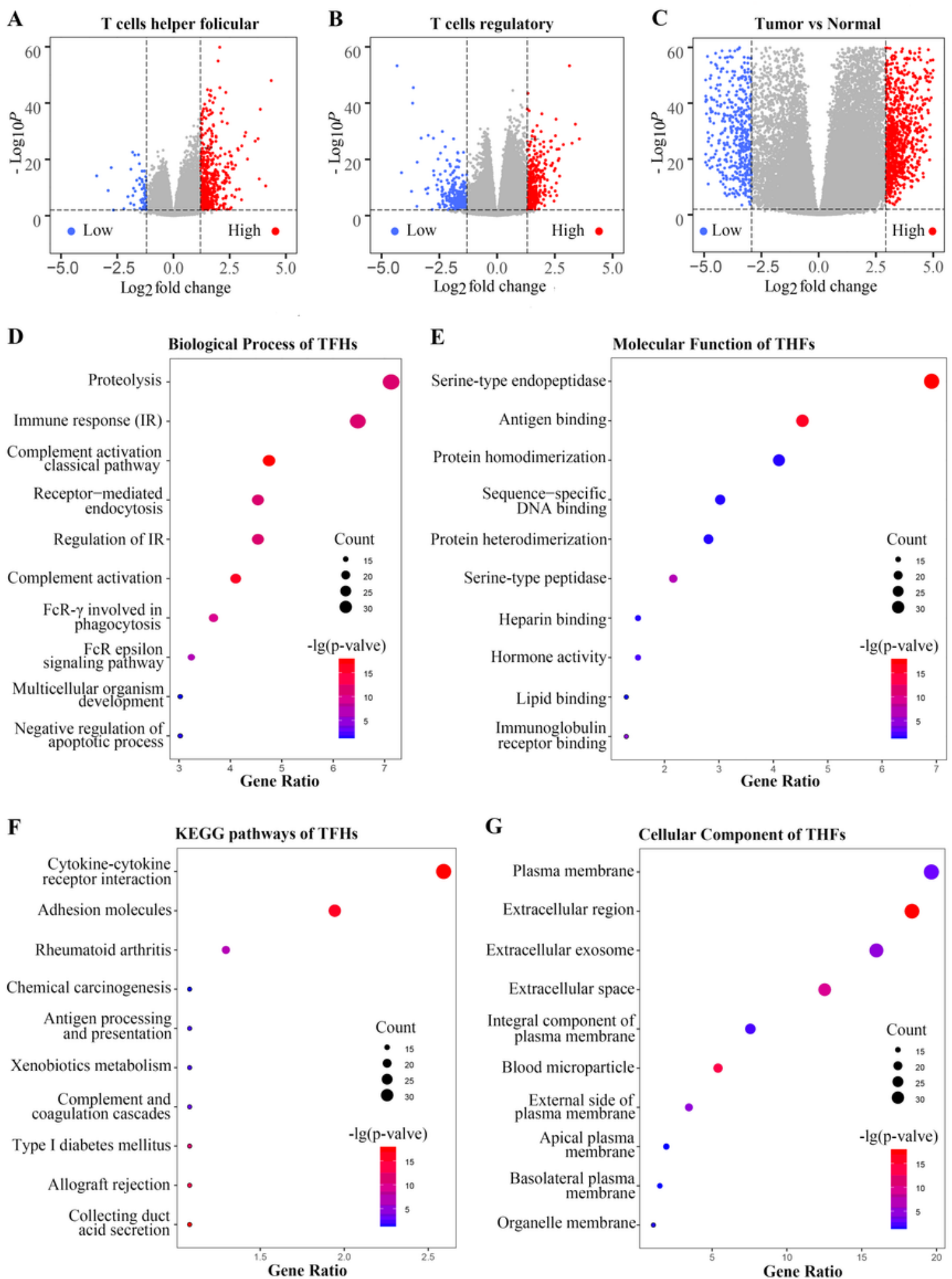

\section{Figure 6}

Identification and Enrichment Analysis of IIRGs and DEGs A The volcano plot shows that difference of gene expression between high TFHs-infiltration group and low TFHs-infiltration group. B The volcano plot shows that difference of gene expression between high Tregs-infiltration group and low Tregs-infiltration group. C DEGs between ccRCC and paracancerous tissues. Each red dot shows an upregulated gene and each green dot shows a downregulated gene. DEGs means different expression genes. D-G the 
enrichment analysis results of IIRGs at biological processes, cellular components, molecular functions, and KEGG levels. The top 10 results of each term are shown, and the color indicates the statistical significance and the size indicates the number of genes enriched for each result. IIRGs is the abbreviation of immune-infiltration-related genes.
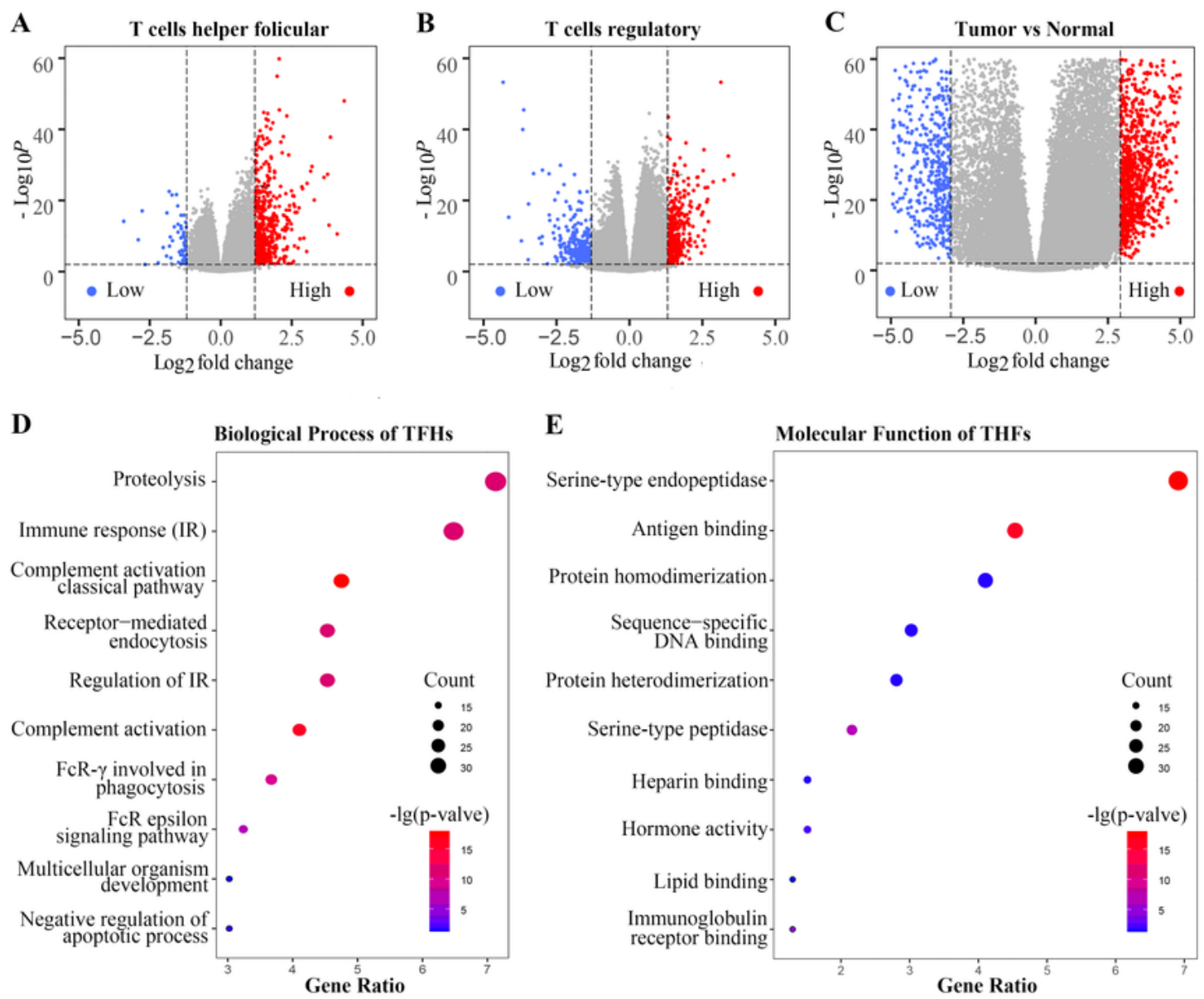

$\mathbf{F}$

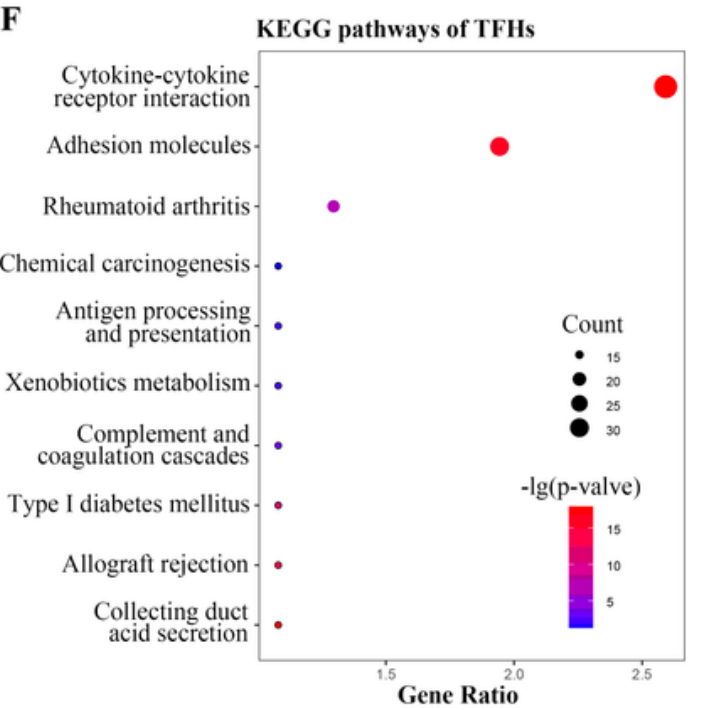

G

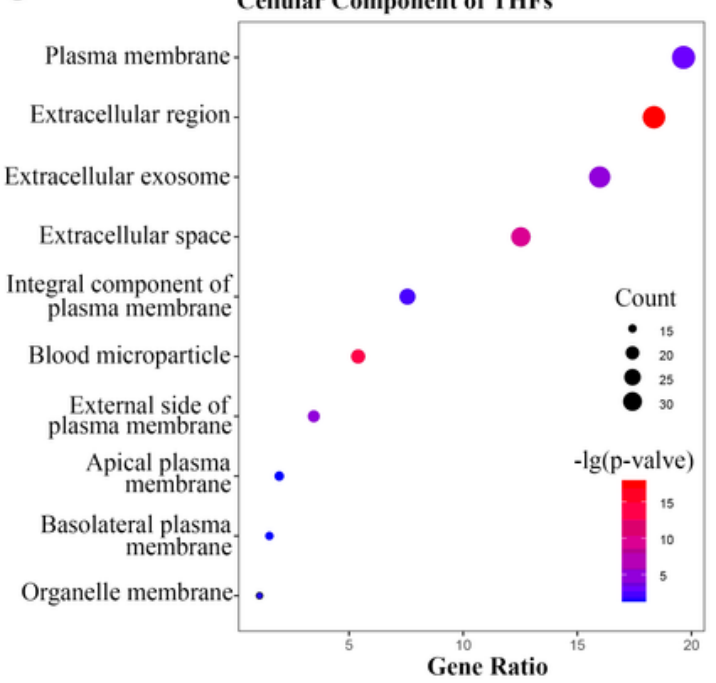

Figure 6 
Identification and Enrichment Analysis of IIRGs and DEGs A The volcano plot shows that difference of gene expression between high TFHs-infiltration group and low TFHs-infiltration group. B The volcano plot shows that difference of gene expression between high Tregs-infiltration group and low Tregs-infiltration group. C DEGs between CCRCC and paracancerous tissues. Each red dot shows an upregulated gene and each green dot shows a downregulated gene. DEGs means different expression genes. D-G the enrichment analysis results of IIRGs at biological processes, cellular components, molecular functions, and KEGG levels. The top 10 results of each term are shown, and the color indicates the statistical significance and the size indicates the number of genes enriched for each result. IIRGs is the abbreviation of immune-infiltration-related genes. 

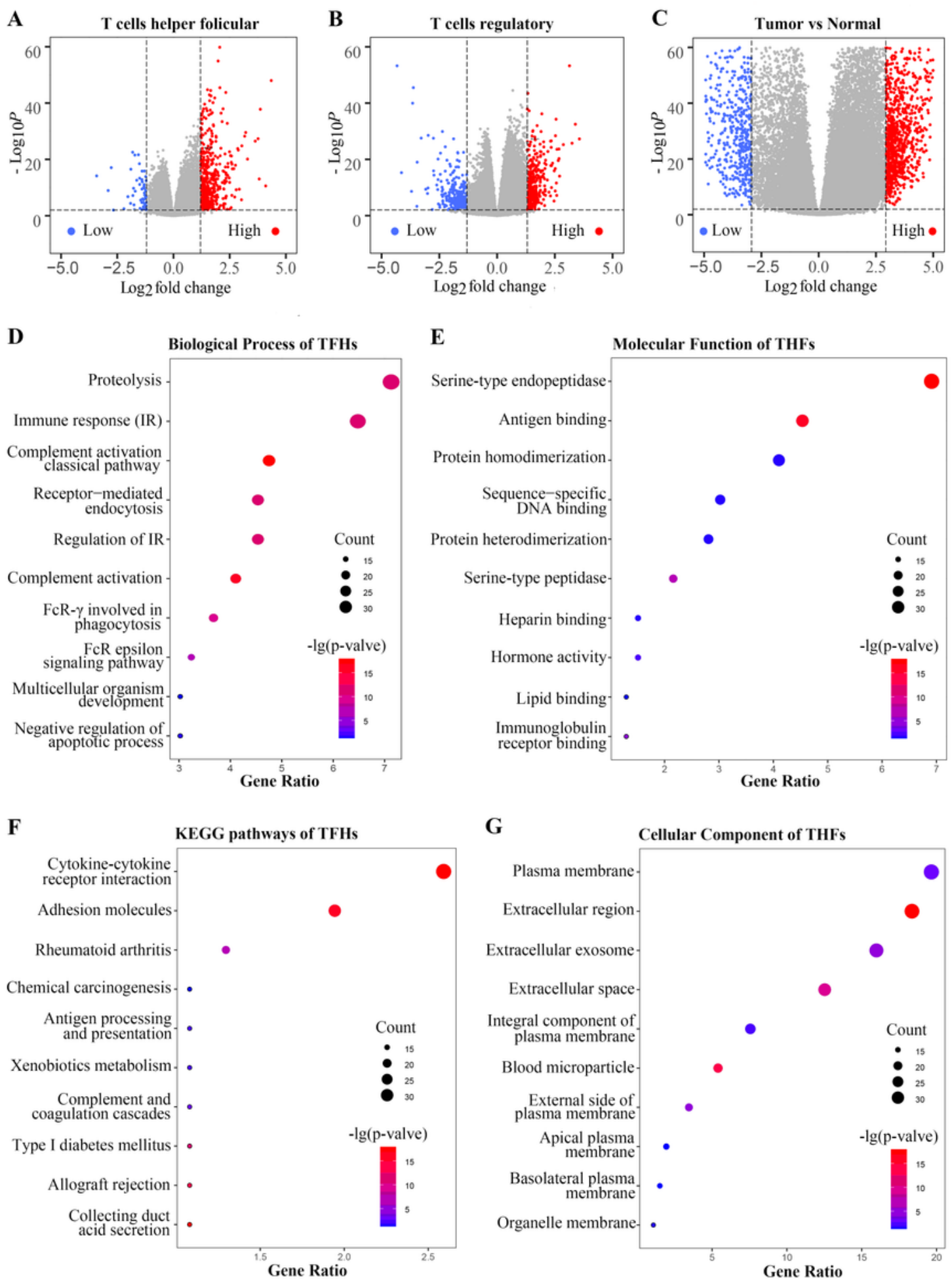

\section{Figure 6}

Identification and Enrichment Analysis of IIRGs and DEGs A The volcano plot shows that difference of gene expression between high TFHs-infiltration group and low TFHs-infiltration group. B The volcano plot shows that difference of gene expression between high Tregs-infiltration group and low Tregs-infiltration group. C DEGs between ccRCC and paracancerous tissues. Each red dot shows an upregulated gene and each green dot shows a downregulated gene. DEGs means different expression genes. D-G the 
enrichment analysis results of IIRGs at biological processes, cellular components, molecular functions, and KEGG levels. The top 10 results of each term are shown, and the color indicates the statistical significance and the size indicates the number of genes enriched for each result. IIRGs is the abbreviation of immune-infiltration-related genes.
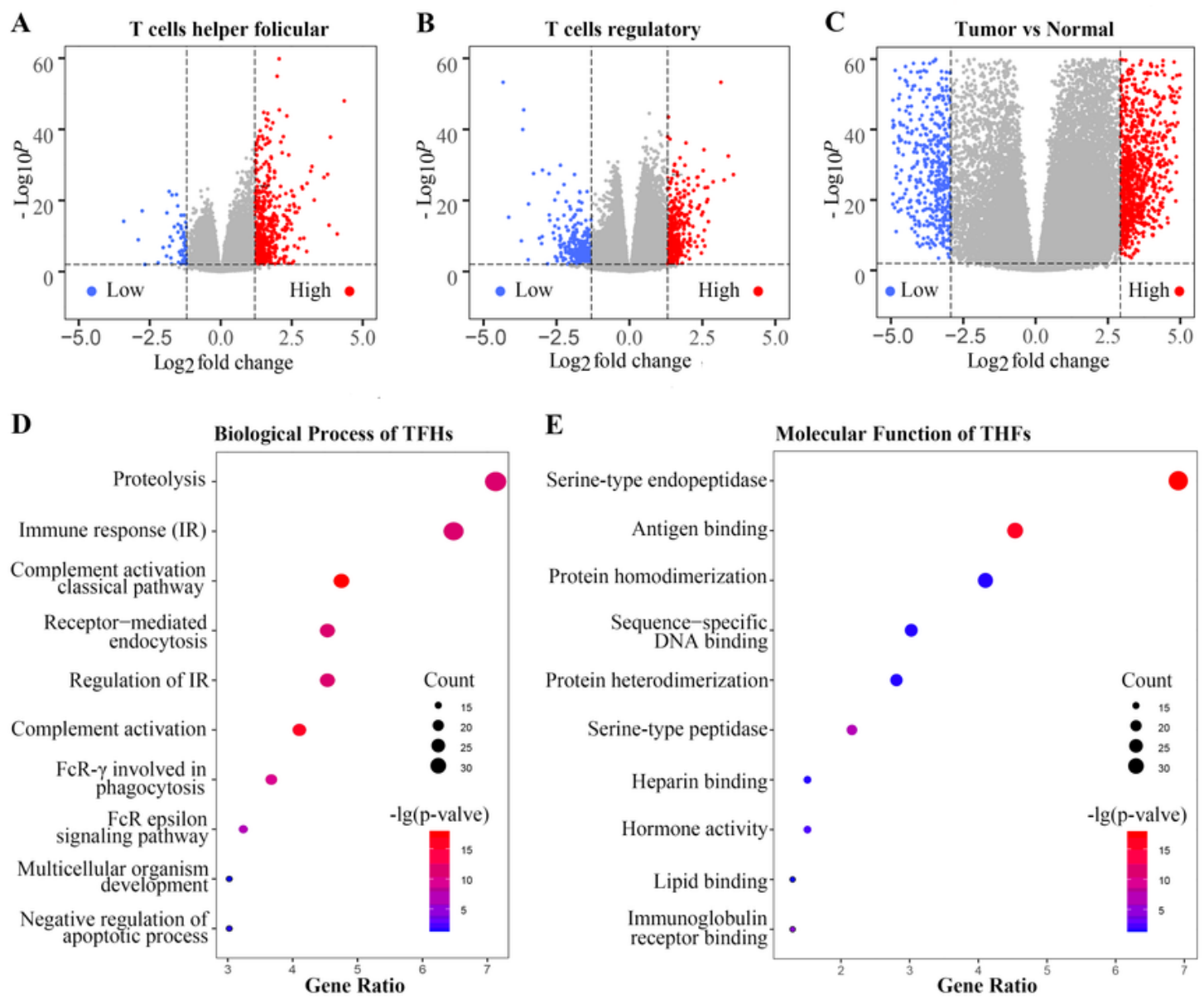

$\mathbf{F}$

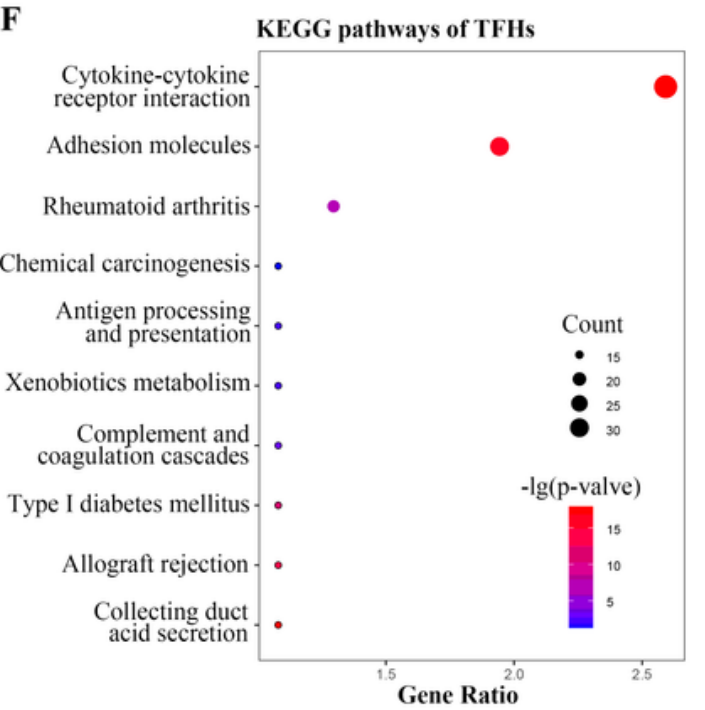

G

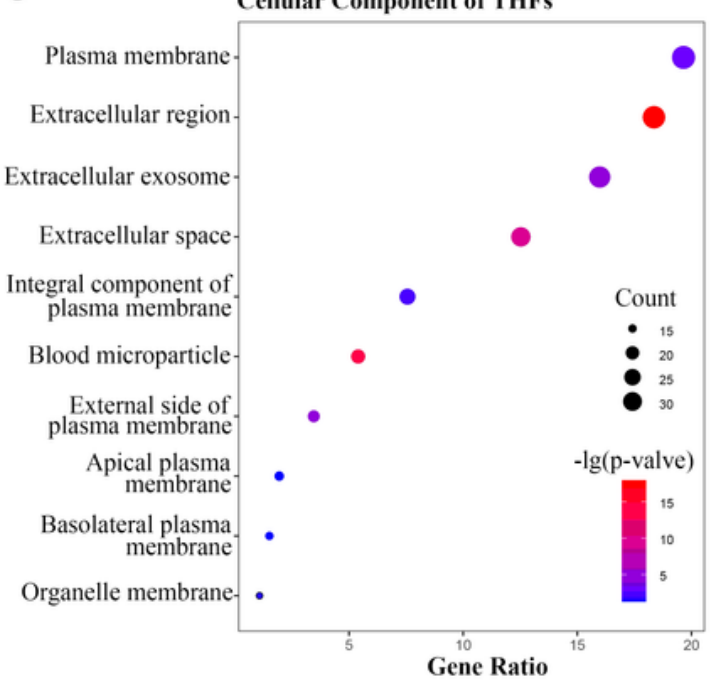

Figure 6 
Identification and Enrichment Analysis of IIRGs and DEGs A The volcano plot shows that difference of gene expression between high TFHs-infiltration group and low TFHs-infiltration group. B The volcano plot shows that difference of gene expression between high Tregs-infiltration group and low Tregs-infiltration group. C DEGs between cCRCC and paracancerous tissues. Each red dot shows an upregulated gene and each green dot shows a downregulated gene. DEGs means different expression genes. D-G the enrichment analysis results of IIRGs at biological processes, cellular components, molecular functions, and KEGG levels. The top 10 results of each term are shown, and the color indicates the statistical significance and the size indicates the number of genes enriched for each result. IIRGs is the abbreviation of immune-infiltration-related genes.

A Intersection of Low IIRGs

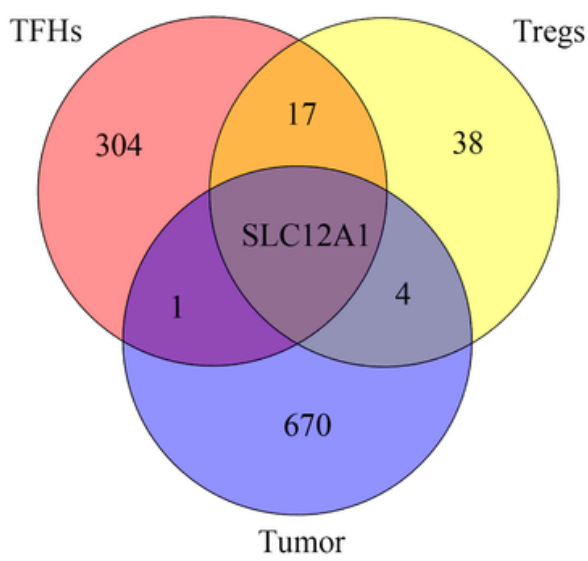

D

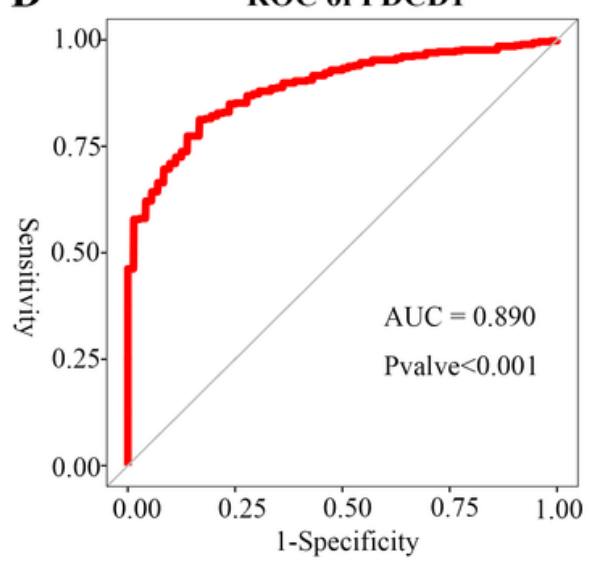

B Intersection of High IIRGs

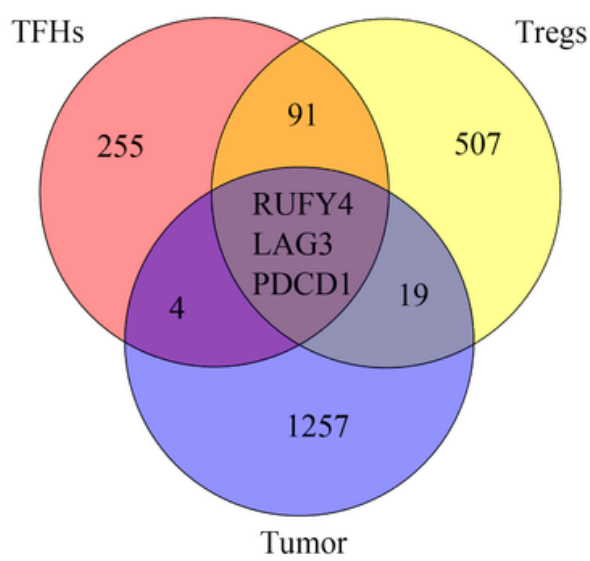

$\mathbf{E}$

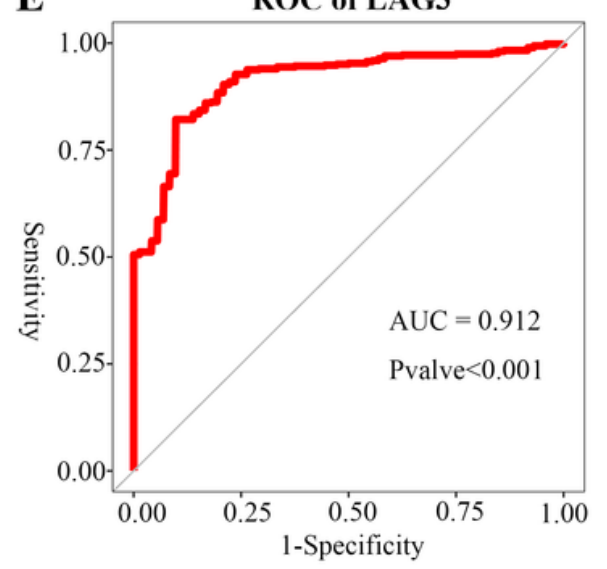

C
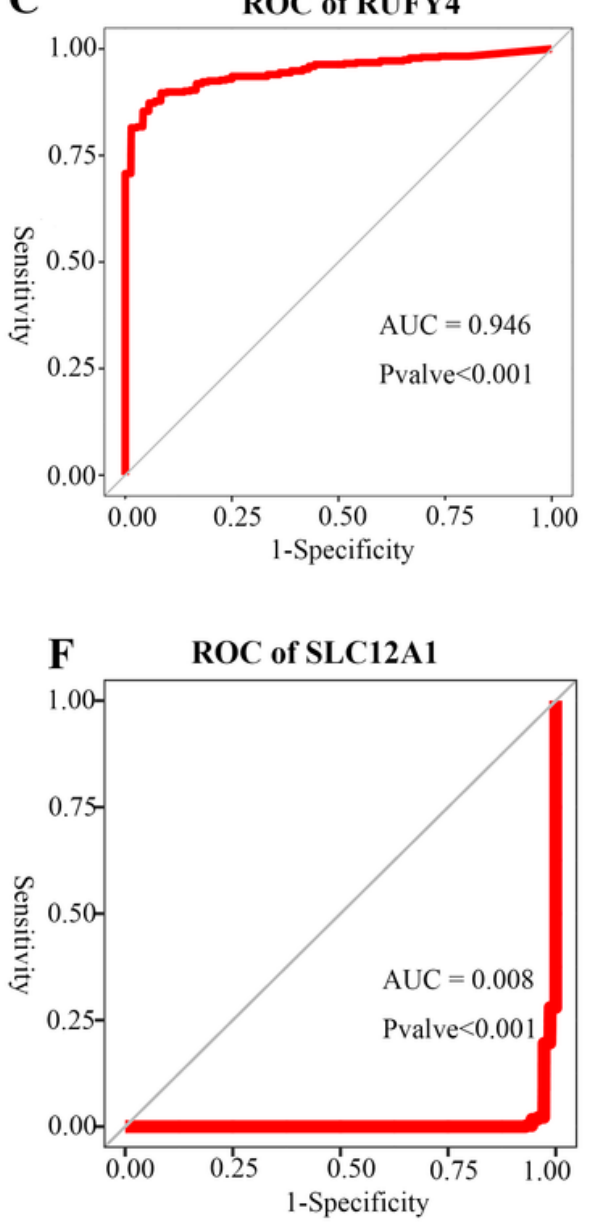

\section{Figure 7}

Identification key genes related to immune-infiltration A Using Venn plot to pick up genes that are lowexpressed in tumors and related to low-density immune-infiltration. B Contrary to A, the figure shows genes that are highly expressed in tumors and related to high-density immune-infiltration of two cells. C-F Receiver operating characteristic (ROC) curve of RUFY4, PDCD1, LAG3 and SCL12A1. ROC curves indicate the capabilities of picking up ccRCC patients from the public. AUC means area under curve. 
A Intersection of Low IIRGs

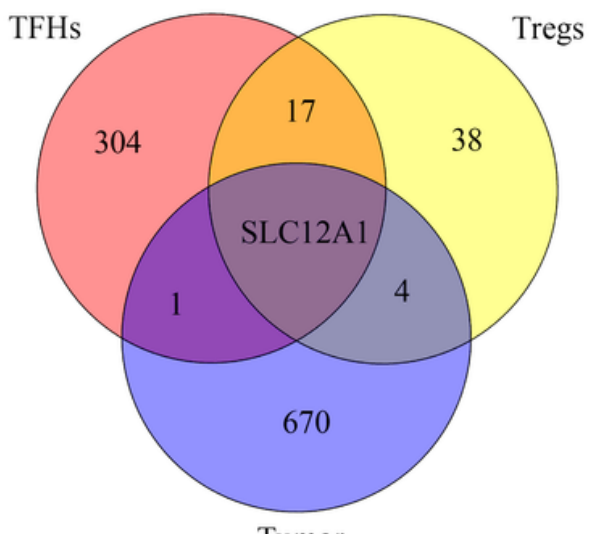

Tumor

D

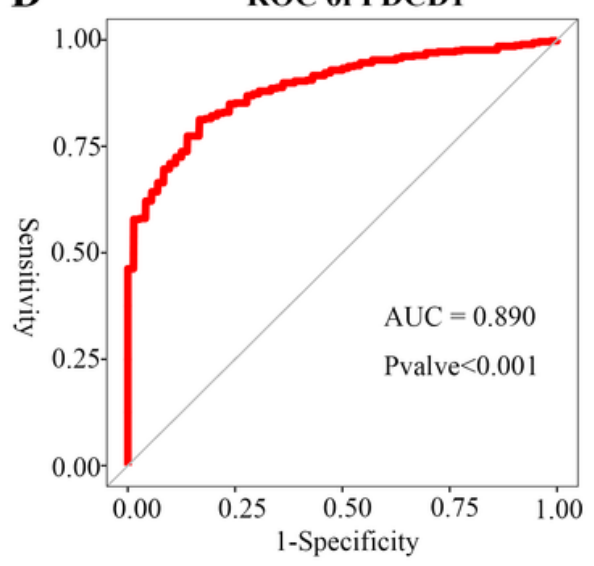

B Intersection of High IIRGs

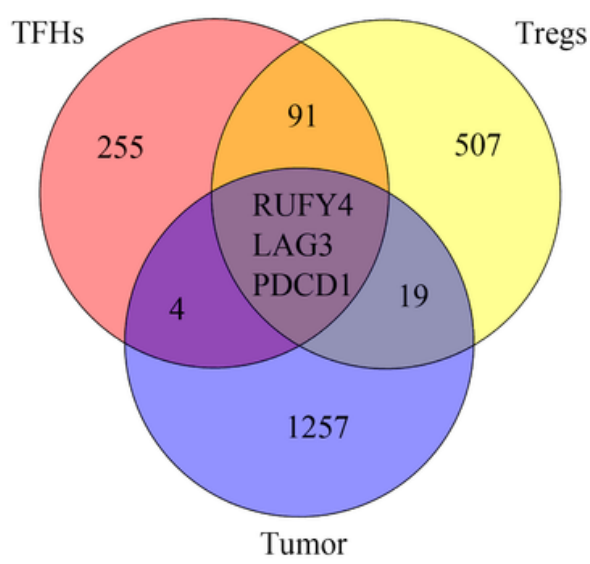

$\mathbf{E}$

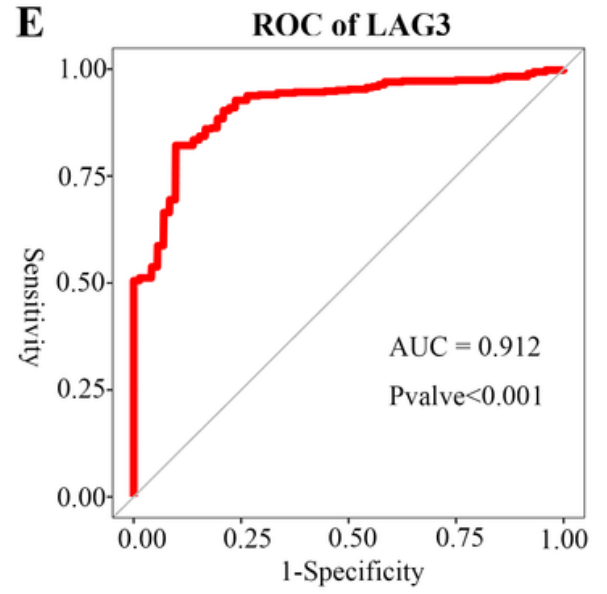

C
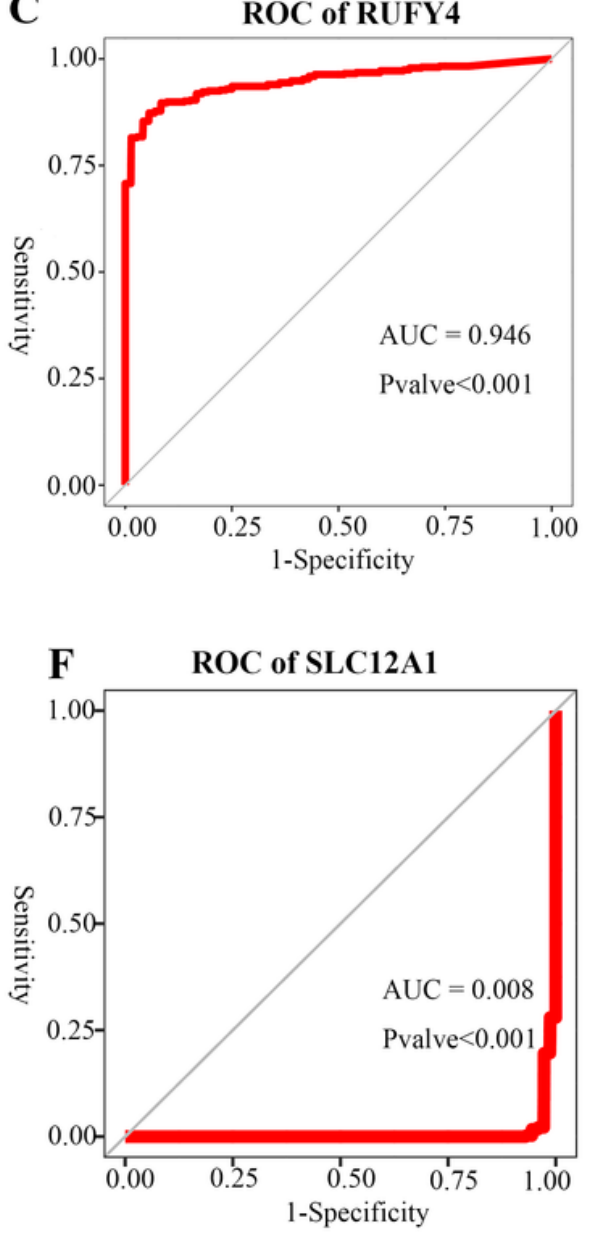

Figure 7

Identification key genes related to immune-infiltration A Using Venn plot to pick up genes that are lowexpressed in tumors and related to low-density immune-infiltration. B Contrary to A, the figure shows genes that are highly expressed in tumors and related to high-density immune-infiltration of two cells. C-F Receiver operating characteristic (ROC) curve of RUFY4, PDCD1, LAG3 and SCL12A1. ROC curves indicate the capabilities of picking up cCRCC patients from the public. AUC means area under curve. 
A Intersection of Low IIRGs

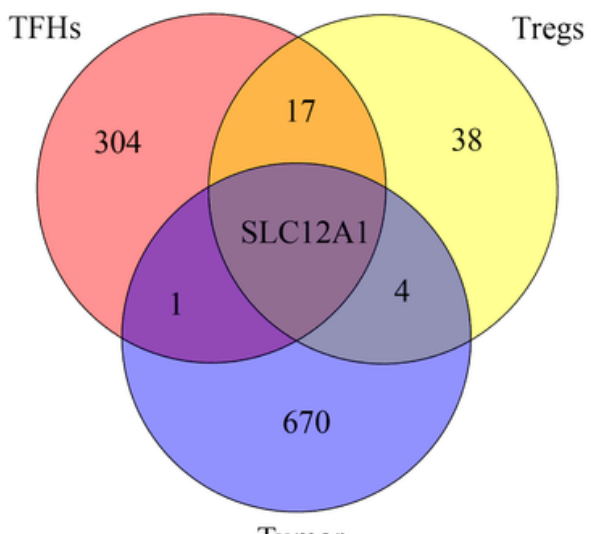

Tumor

D

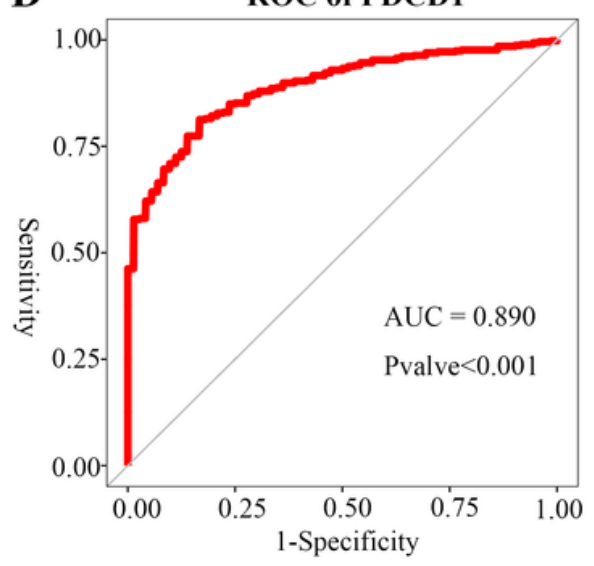

B Intersection of High IIRGs

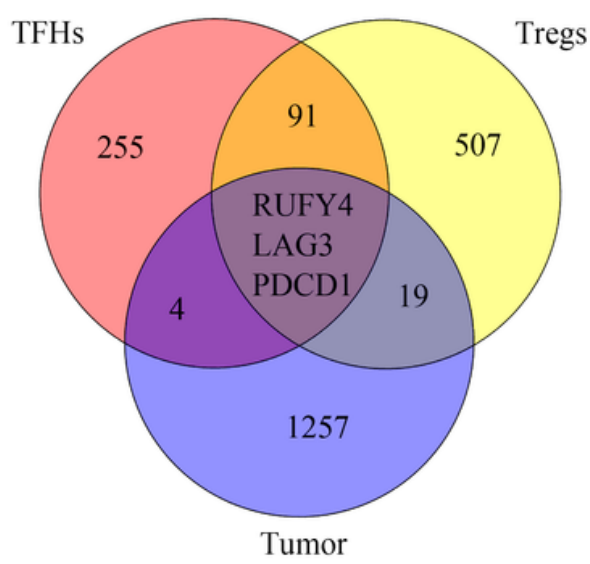

$\mathbf{E}$

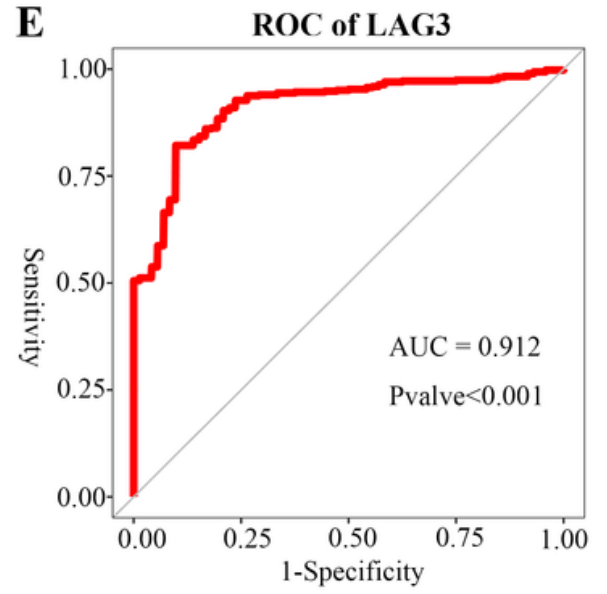

C
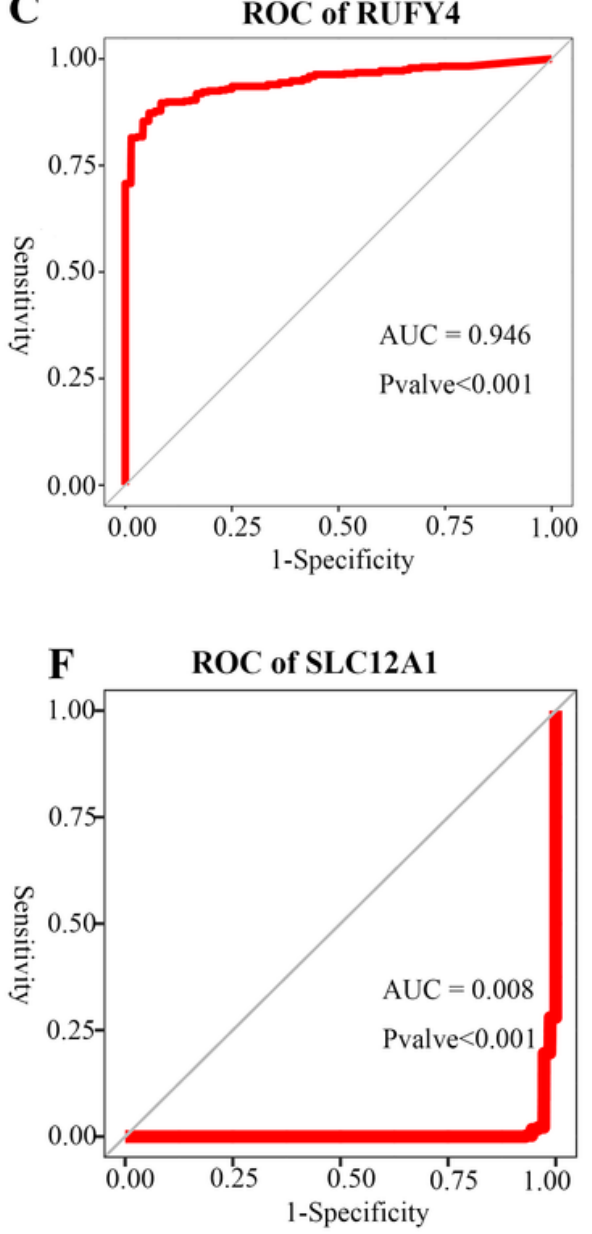

Figure 7

Identification key genes related to immune-infiltration A Using Venn plot to pick up genes that are lowexpressed in tumors and related to low-density immune-infiltration. B Contrary to A, the figure shows genes that are highly expressed in tumors and related to high-density immune-infiltration of two cells. C-F Receiver operating characteristic (ROC) curve of RUFY4, PDCD1, LAG3 and SCL12A1. ROC curves indicate the capabilities of picking up cCRCC patients from the public. AUC means area under curve. 
A Intersection of Low IIRGs

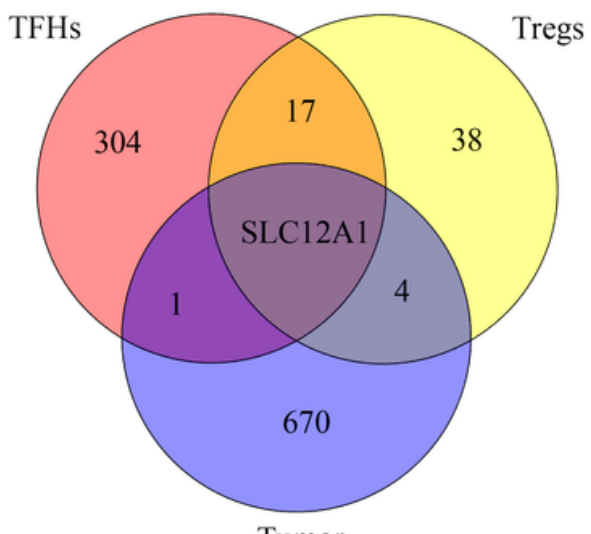

Tumor

D

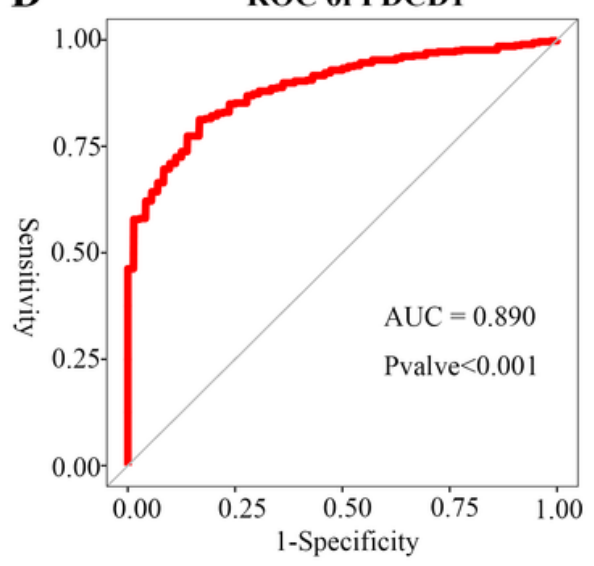

B Intersection of High IIRGs

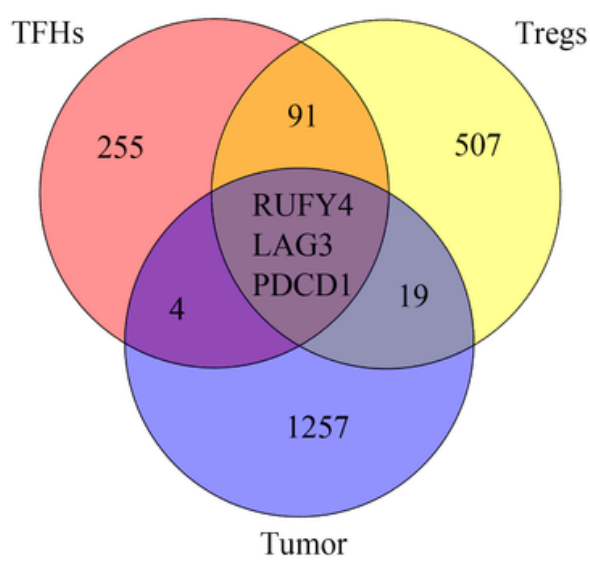

$\mathbf{E}$

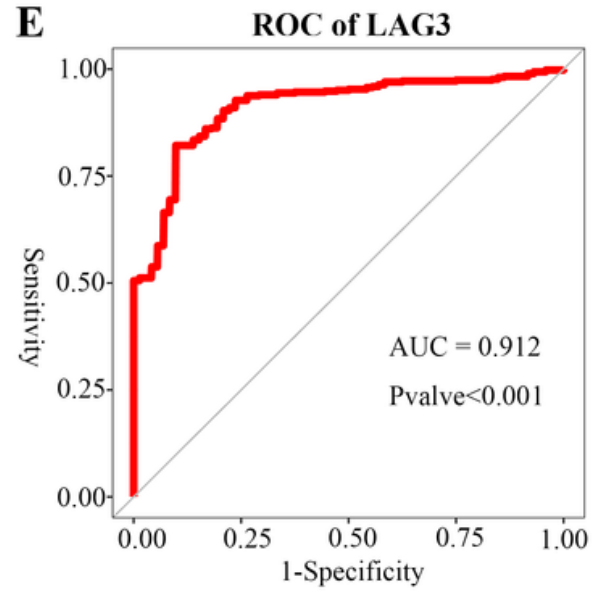

C
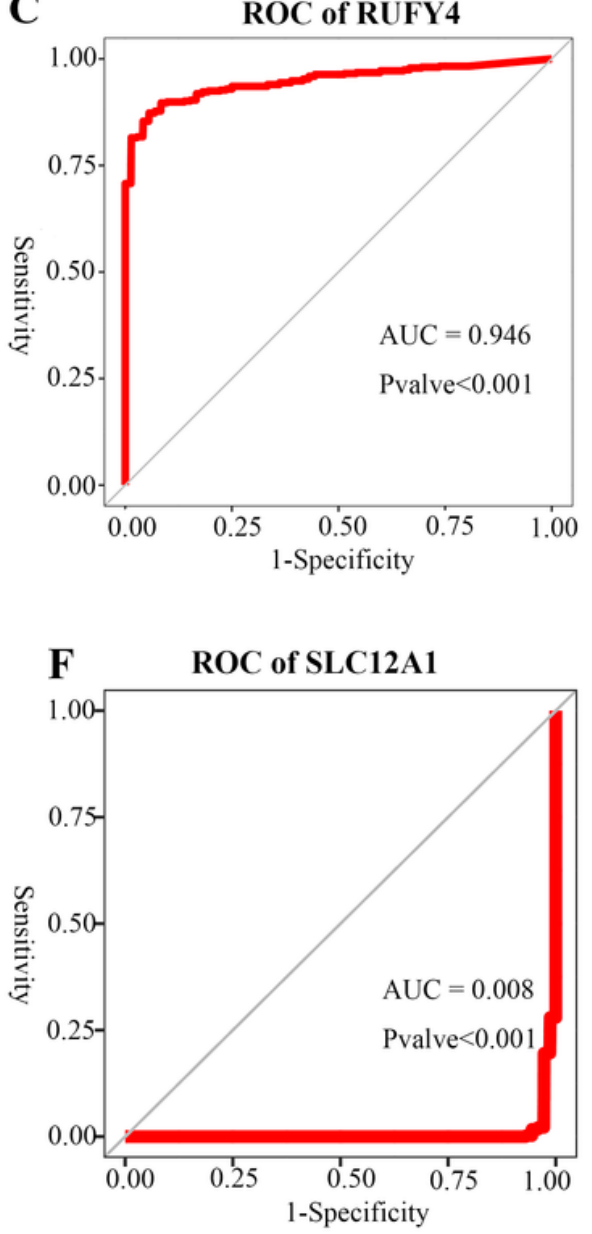

Figure 7

Identification key genes related to immune-infiltration A Using Venn plot to pick up genes that are lowexpressed in tumors and related to low-density immune-infiltration. B Contrary to A, the figure shows genes that are highly expressed in tumors and related to high-density immune-infiltration of two cells. C-F Receiver operating characteristic (ROC) curve of RUFY4, PDCD1, LAG3 and SCL12A1. ROC curves indicate the capabilities of picking up cCRCC patients from the public. AUC means area under curve. 
A

Corelations between co-expressed genes

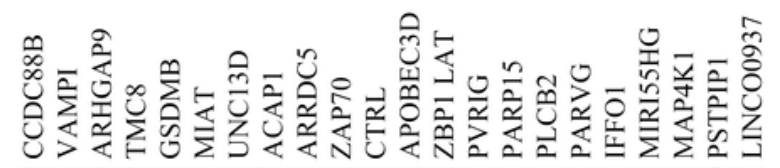

CCDC88B $0000000000000 \cdot 00000000$

VAMPI 0000000000000000000000 ARHGAP9 0000000000000000000000

TMC8 0000000000000000000

GSDMB 0000000000000000000000

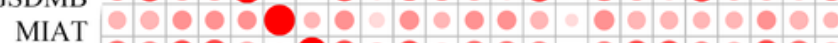

UNC13D 80800080000000000000800

ACAP1 100000

ARRDC5 0000000000000000000

ZAP70

CTRL 0000000000000000000000 APOBEC3D ZBP1 LAT 0000000000000000000000

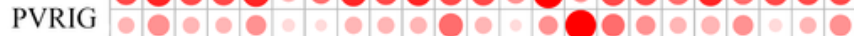

PARP15 00000000000000000000000

PLCB2 OOOOO०00000000000000000

PARVG 00000000000000000000

IFFO1 0000000000000000000000 MIRI55HG 0000000000000000000000 MAP4K1 00000000000000000000 PSTPIP1 00000000000000000000 LINCO0937 OOOOO०00000000000000000

B

Pathways of RUF4 from ClueGo

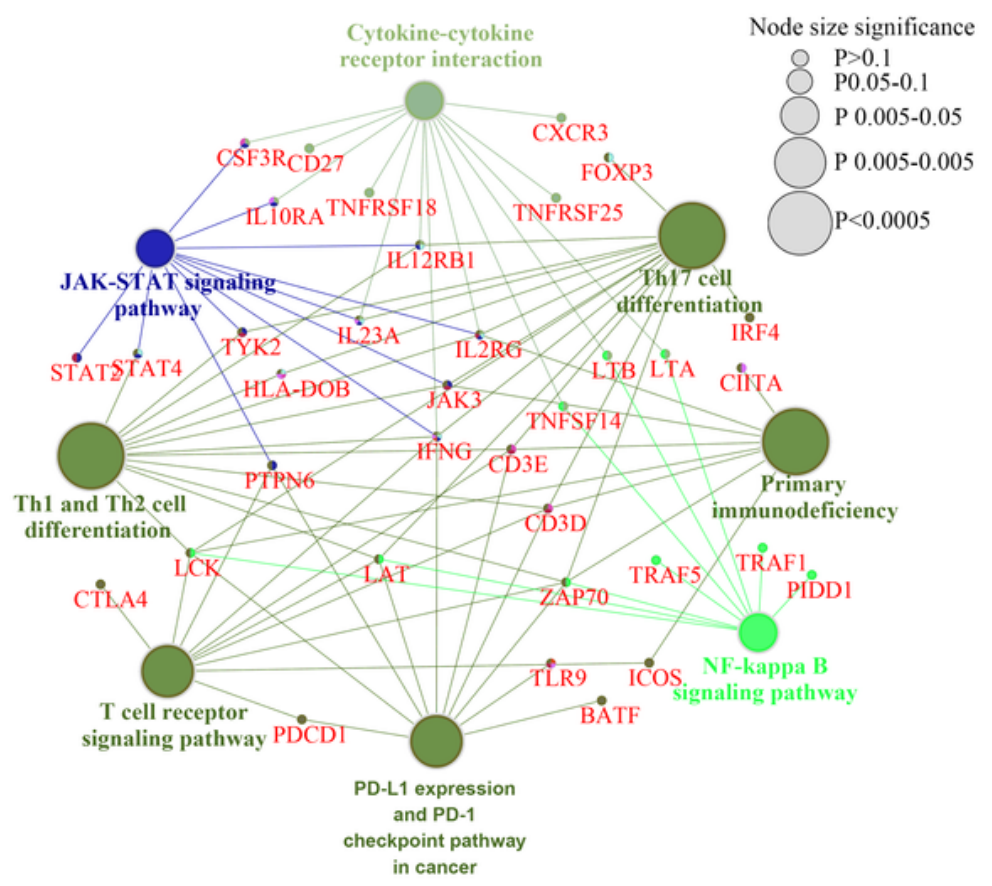

C TCR signaling pathaway

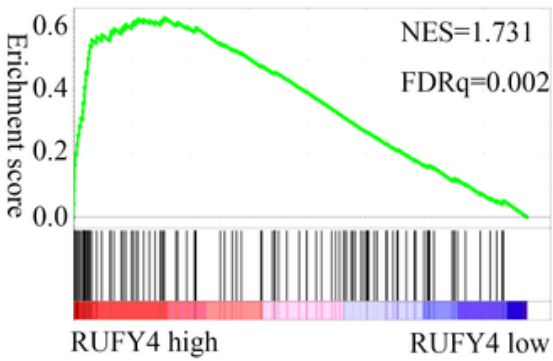

D Cytokine cytokine receptor interaction
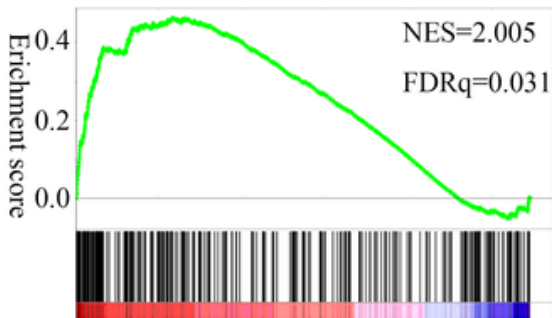

RUFY4 high

RUFY4 low

E Cancer immunotherapy PD1 blockade

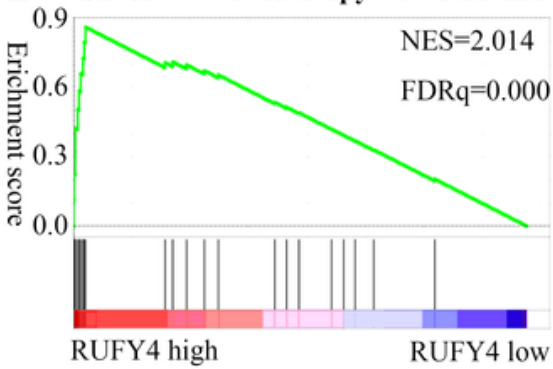

F TCR and constimulatory signaling

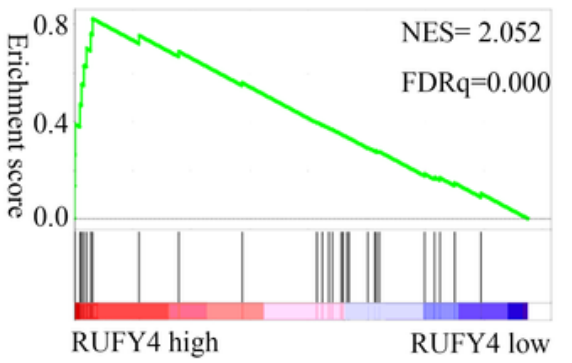

\section{Figure 8}

Identification key genes related to immune-infiltration A Using Venn plot to pick up genes that are lowexpressed in tumors and related to low-density immune-infiltration. B Contrary to A, the figure shows genes that are highly expressed in tumors and related to high-density immune-infiltration of two cells. C-F Receiver operating characteristic (ROC) curve of RUFY4, PDCD1, LAG3 and SCL12A1. ROC curves indicate the capabilities of picking up ccRCC patients from the public. AUC means area under curve. 
A

Corelations between co-expressed genes

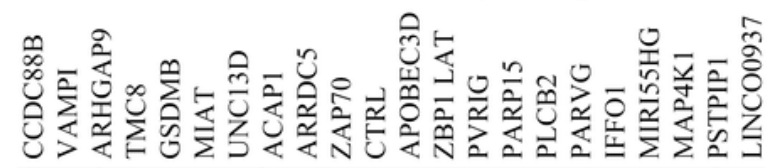

CCDC88B $0000000000000 \cdot 00000000$

VAMPI 0000000000000000000000 ARHGAP9 0000000000000000000000

TMC8 0000000000000000000

GSDMB 0000000000000000000000

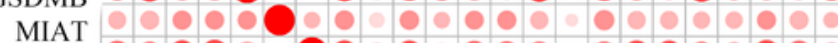

UNC13D 80800080000000000000800

ACAP1 100000

ARRDC5 0000000000000000000

ZAP70

CTRL 0000000000000000000000 APOBEC3D ZBP1 LAT 0000000000000000000000

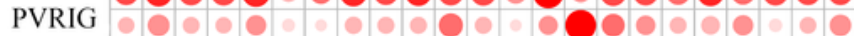

PARP15 00000000000000000000000

PLCB2 OOOOO०00000000000000000

PARVG 00000000000000000000

IFFO1 0000000000000000000000 MIRI55HG 0000000000000000000000 MAP4K1 00000000000000000000 PSTPIP1 00000000000000000000 LINCO0937 OOOOO०00000000000000000

B

Pathways of RUF4 from ClueGo

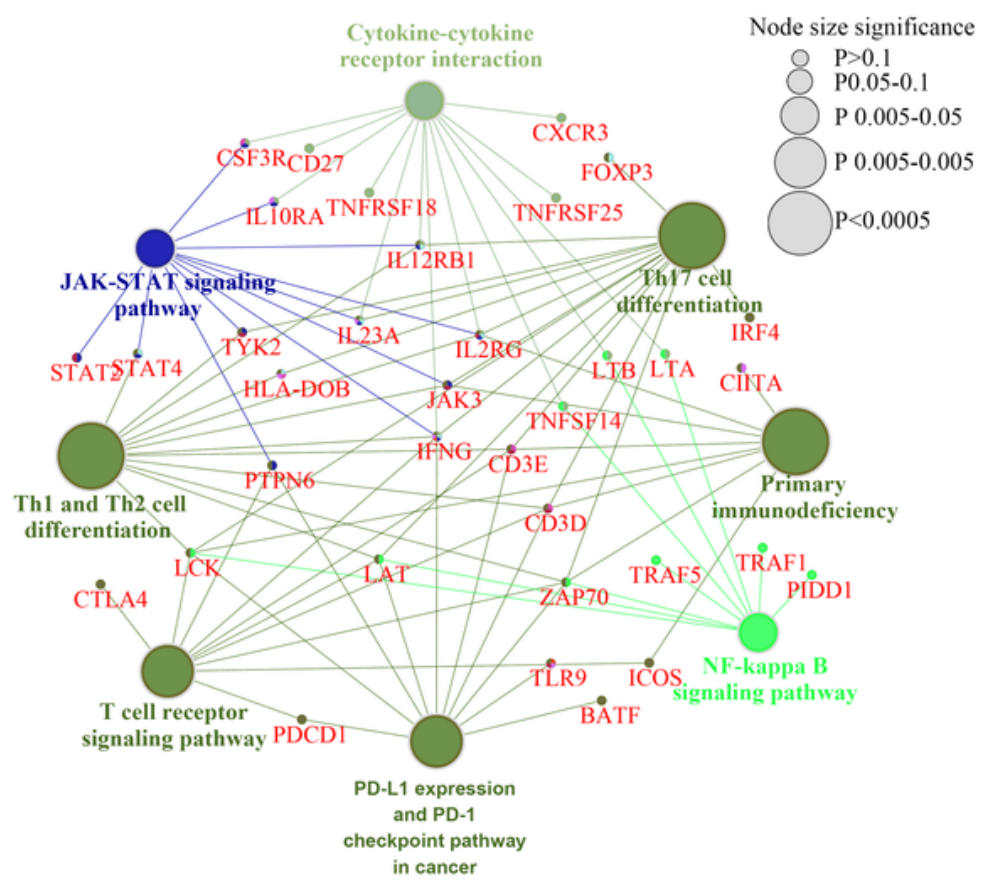

C TCR signaling pathaway

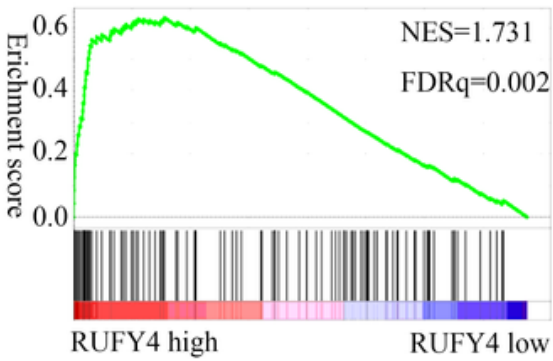

D Cytokine cytokine receptor interaction
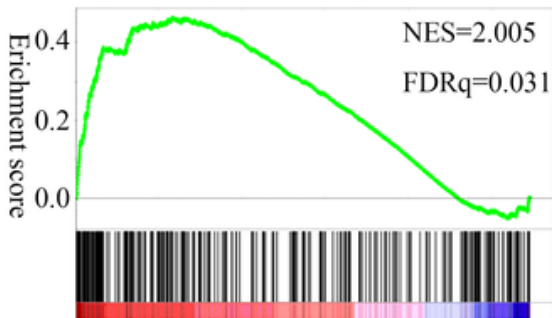

RUFY4 high

RUFY4 low

E Cancer immunotherapy PD1 blockade

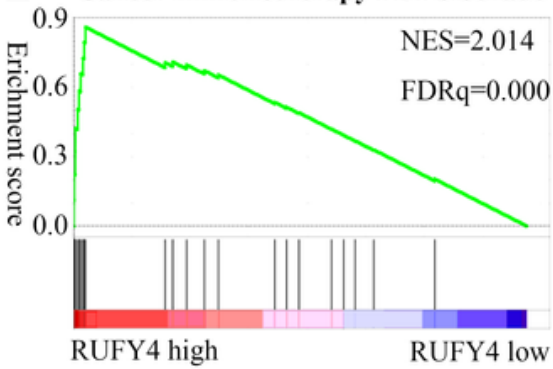

F TCR and constimulatory signaling

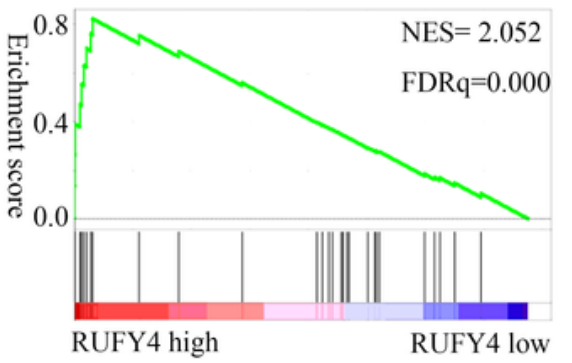

\section{Figure 8}

Identification key genes related to immune-infiltration A Using Venn plot to pick up genes that are lowexpressed in tumors and related to low-density immune-infiltration. B Contrary to A, the figure shows genes that are highly expressed in tumors and related to high-density immune-infiltration of two cells. C-F Receiver operating characteristic (ROC) curve of RUFY4, PDCD1, LAG3 and SCL12A1. ROC curves indicate the capabilities of picking up ccRCC patients from the public. AUC means area under curve. 
A

Corelations between co-expressed genes

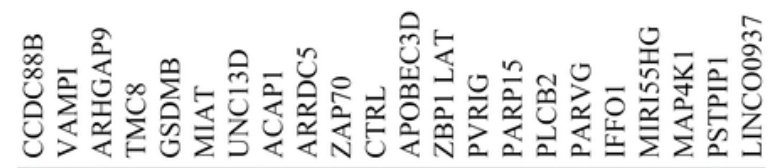

CCDC88B 0000000000000.00000000

VAMPI 0000000000000000000000 ARHGAP9 000000000000000000000

TMC8 00000000000000000000 Pvalve

GSDMB $000000000000000000000 \div 0.05$

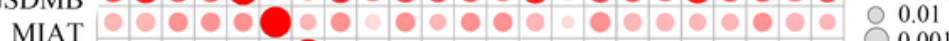

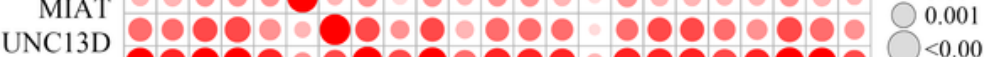

ACAP1 00000000000000000000000

ARRDC5

ZAP70 000000000000000000000

CTRL $00000 \circ 00000000000000000$ APOBEC3D 90900000000000000000000 ZBP1 LAT 0000000000000000000000 PVRIG PARP15 0000000000000000000000 PLCB2 00000000000000000000 PARVG 000000000000000000000

IFFO1 0000000000000000000000

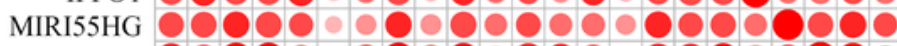
MAP4K1 OOOOO-00000000 0000000

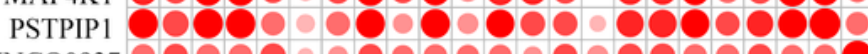
LINCO093700000०00000000000000000

B Pathways of RUF4 from ClueGO

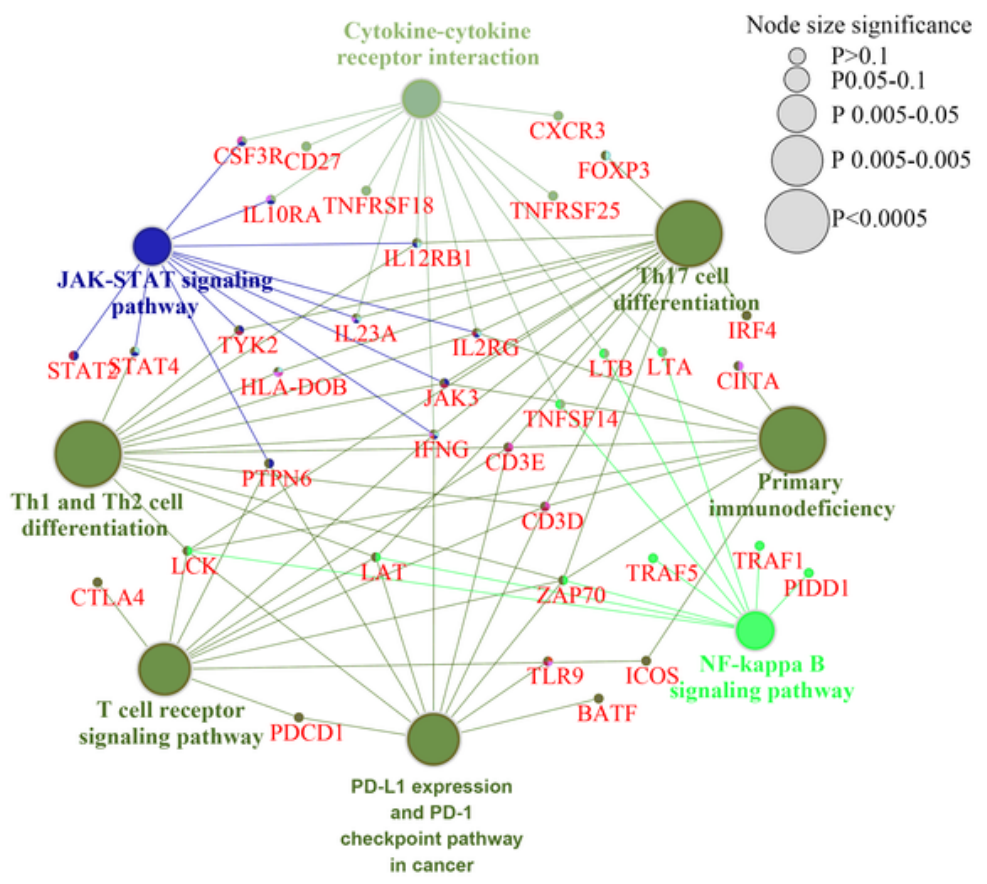

C TCR signaling pathaway

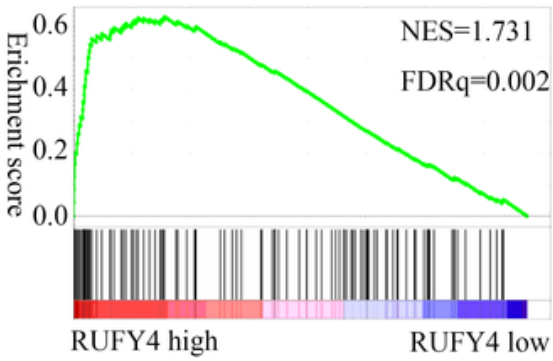

D Cytokine cytokine receptor interaction
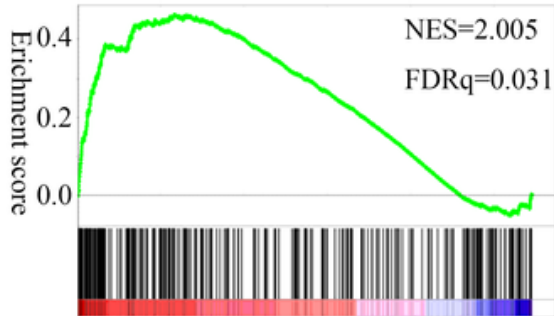

RUFY4 high

RUFY4 low

E Cancer immunotherapy PD1 blockade

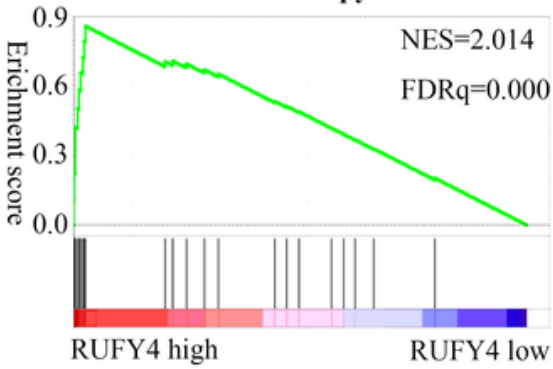

F TCR and constimulatory signaling

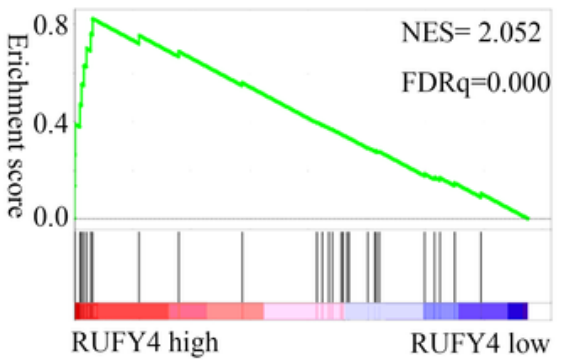

\section{Figure 8}

Identification key genes related to immune-infiltration A Using Venn plot to pick up genes that are lowexpressed in tumors and related to low-density immune-infiltration. B Contrary to A, the figure shows genes that are highly expressed in tumors and related to high-density immune-infiltration of two cells. C-F Receiver operating characteristic (ROC) curve of RUFY4, PDCD1, LAG3 and SCL12A1. ROC curves indicate the capabilities of picking up ccRCC patients from the public. AUC means area under curve. 
A

Corelations between co-expressed genes

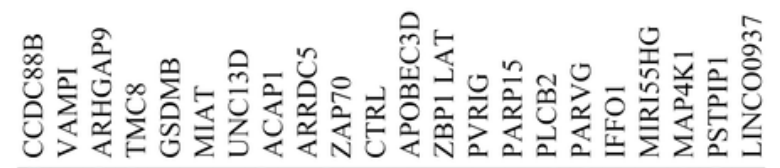

CCDC88B 0000000000000.00000000

VAMPI 0000000000000000000000 ARHGAP9 000000000000000000000

TMC8 00000000000000000000 Pvalve

GSDMB $000000000000000000000 \div 0.05$

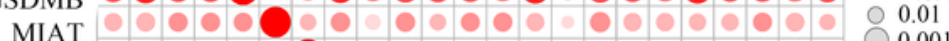

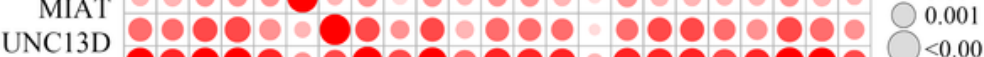

ACAP1 00000000000000000000000

ARRDC5

ZAP70 000000000000000000000

CTRL $00000 \circ 00000000000000000$ APOBEC3D 90900000000000000000000 ZBP1 LAT 0000000000000000000000 PVRIG PARP15 0000000000000000000000 PLCB2 00000000000000000000 PARVG 000000000000000000000

IFFO1 0000000000000000000000

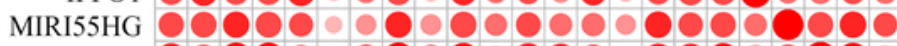
MAP4K1 OOOOO-00000000 0000000

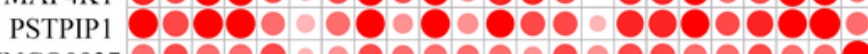
LINCO093700000०00000000000000000

B Pathways of RUF4 from ClueGO

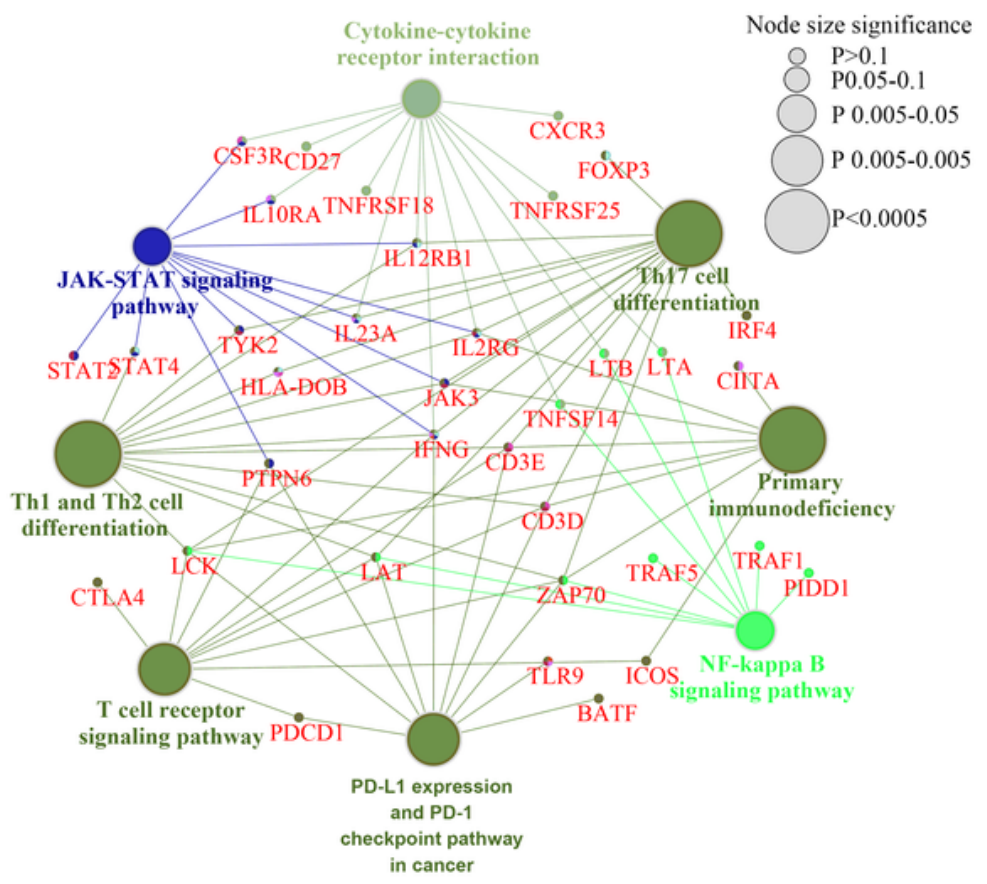

C TCR signaling pathaway

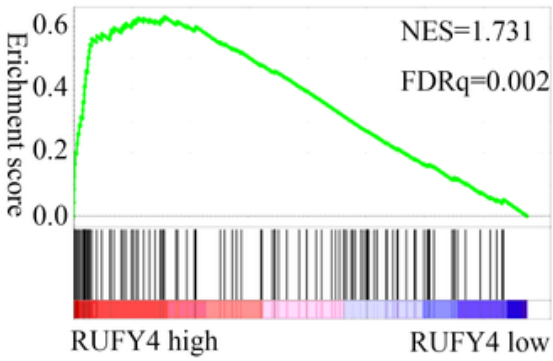

D Cytokine cytokine receptor interaction
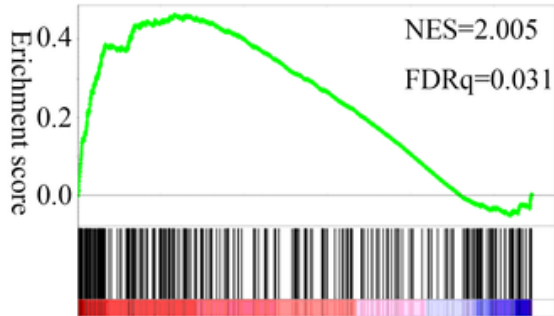

RUFY4 high

RUFY4 low

E Cancer immunotherapy PD1 blockade

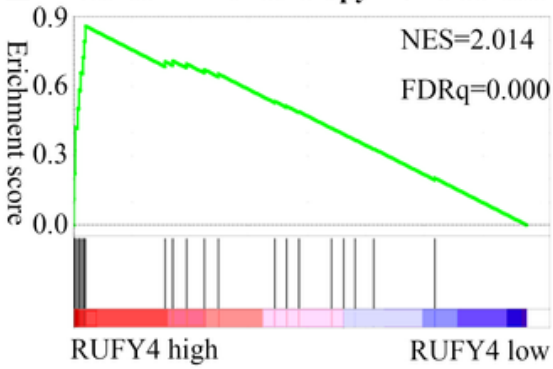

F TCR and constimulatory signaling

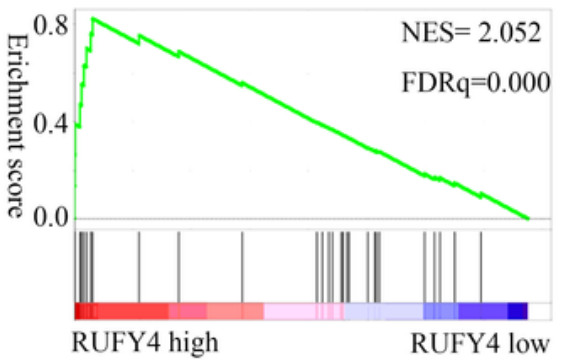

\section{Figure 8}

Identification key genes related to immune-infiltration A Using Venn plot to pick up genes that are lowexpressed in tumors and related to low-density immune-infiltration. B Contrary to A, the figure shows genes that are highly expressed in tumors and related to high-density immune-infiltration of two cells. C-F Receiver operating characteristic (ROC) curve of RUFY4, PDCD1, LAG3 and SCL12A1. ROC curves indicate the capabilities of picking up ccRCC patients from the public. AUC means area under curve. 
A

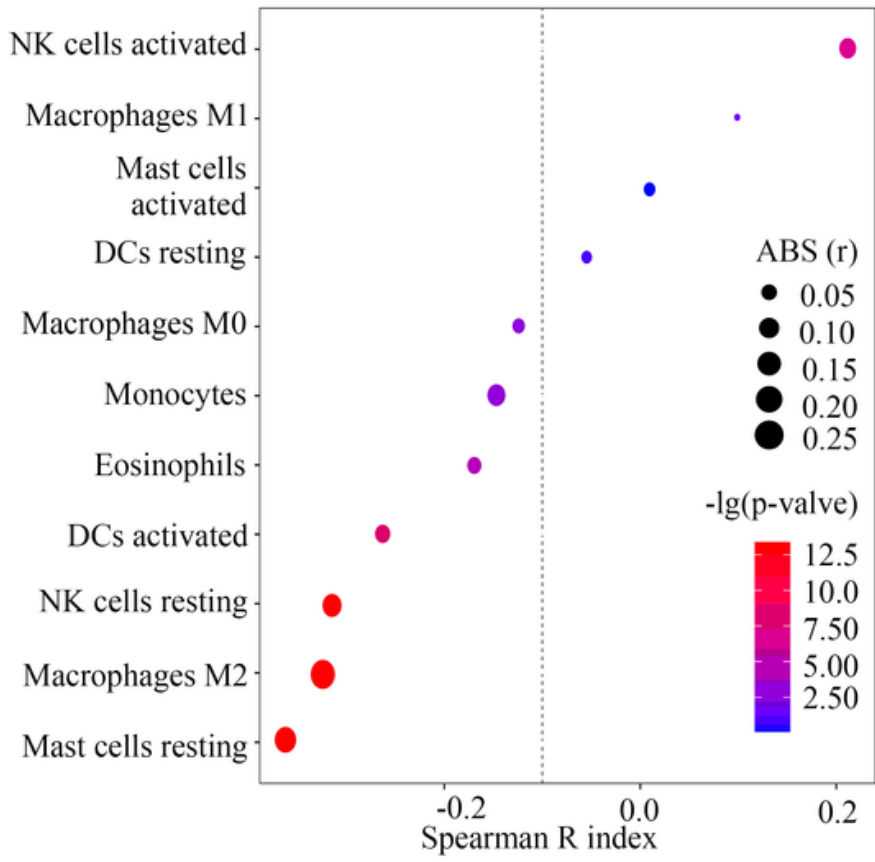

B

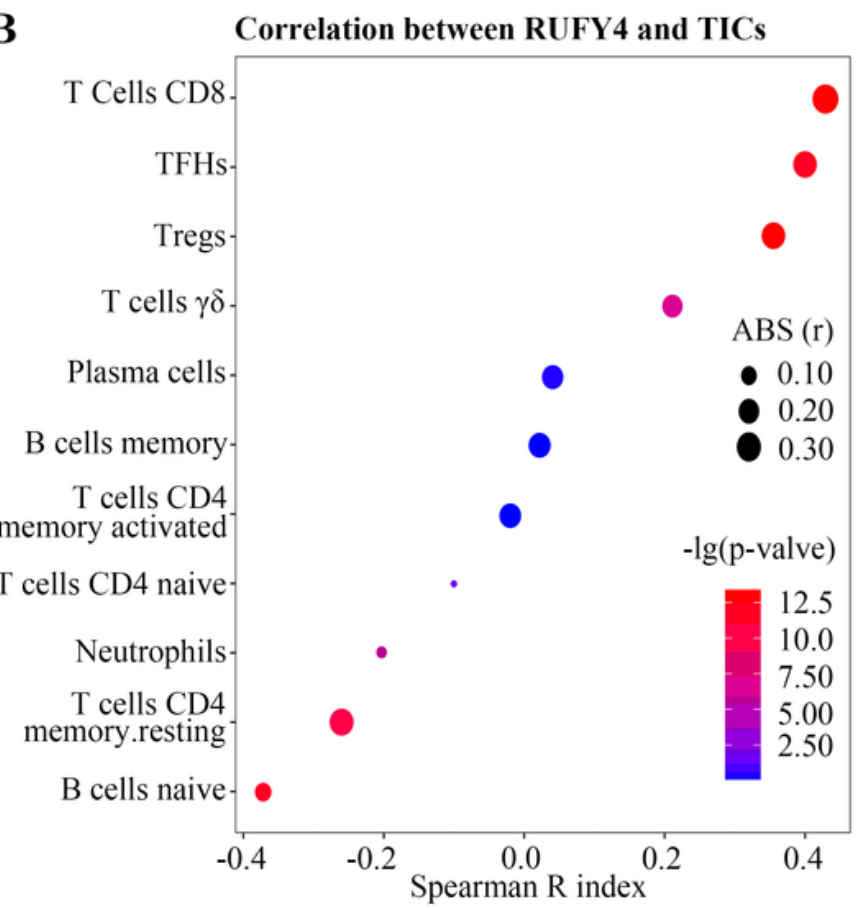

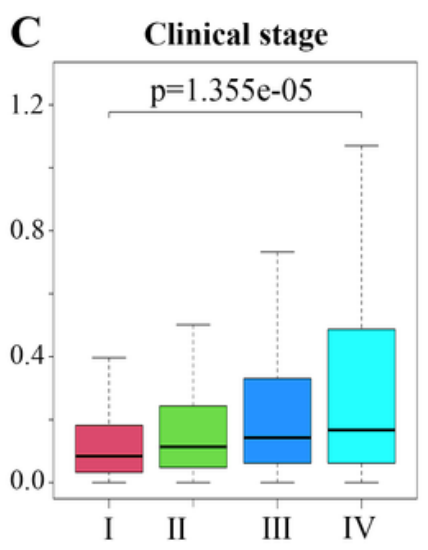

D Pathological stage
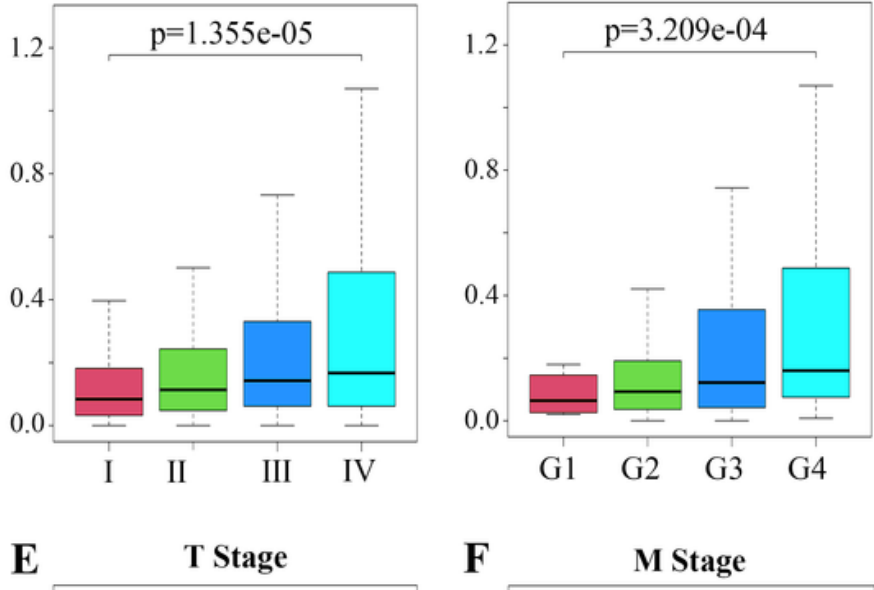

F M Stage
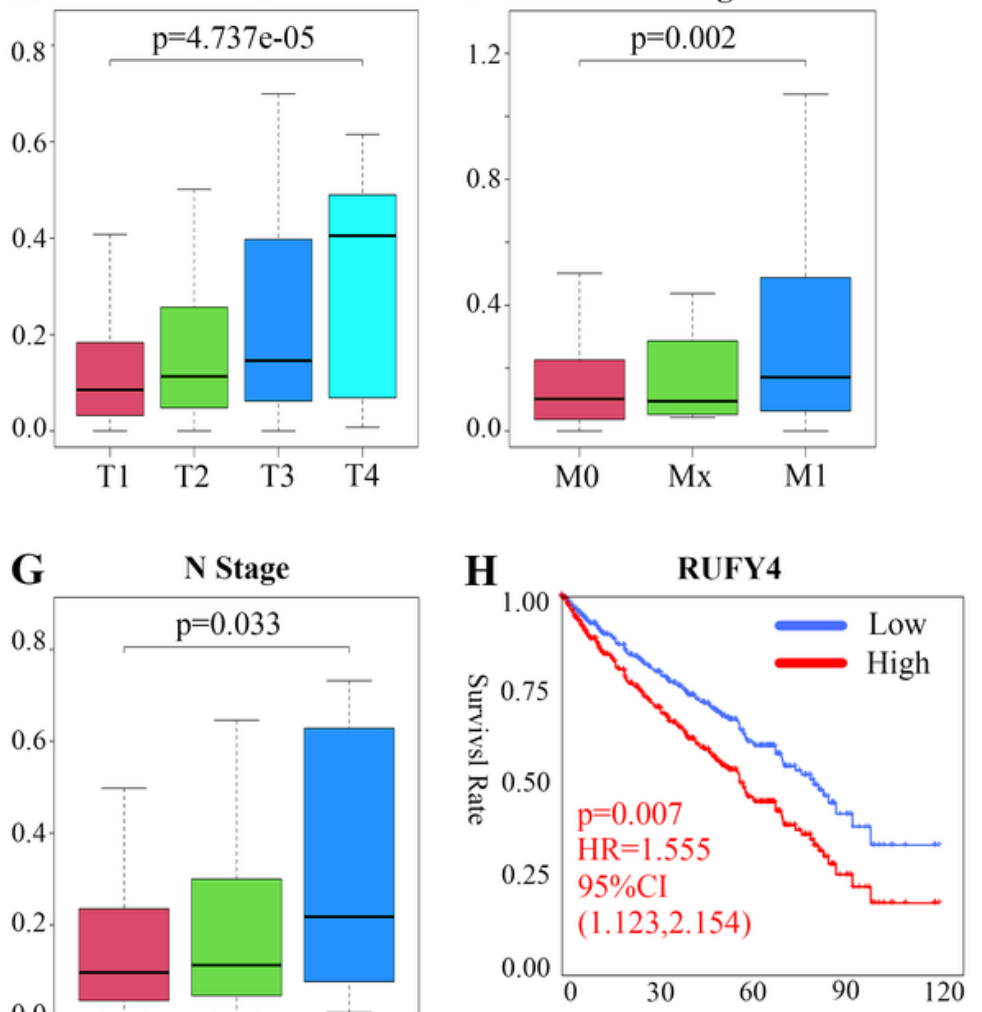

\section{Figure 9}

Validation of the immune correlation and prognostic valve A-B The correlation between RUFY4 and 22 immune cells. The color of each point represented the degree of statistical significance while the size and position of each point represent the correlation coefficient of person and spearman respectively. C-G The relationship between the expression level of RUFY4 and clinical stage, pathological stage, $T$ stage, M stage and $\mathrm{N}$ stage. The ordinate represents the content of immune cells and the horizontal line inside the 
box represents the median value of immune cell contents. $\mathrm{H}$ The multivariate survival analysis for RUFY4 after adjusting known risk factors.
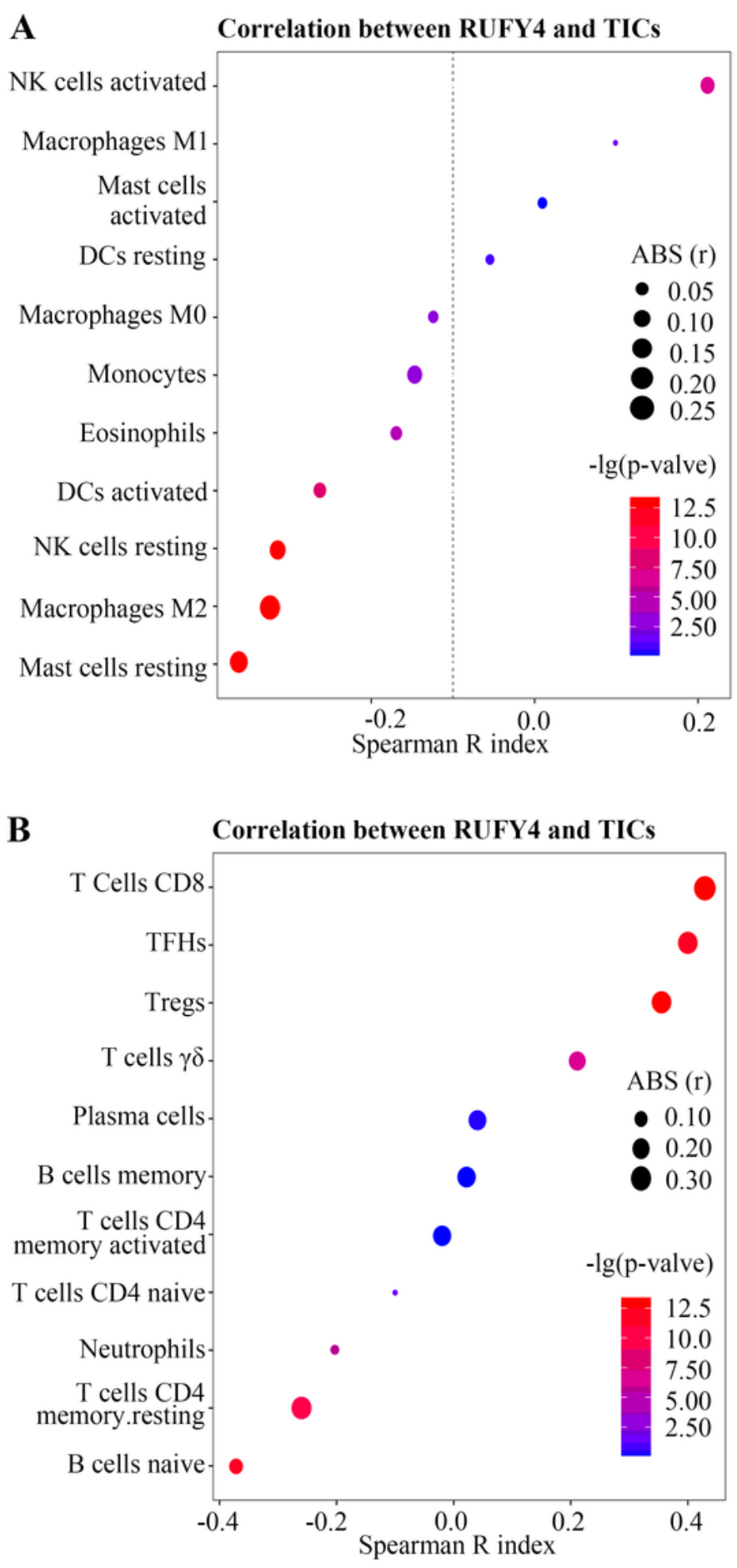
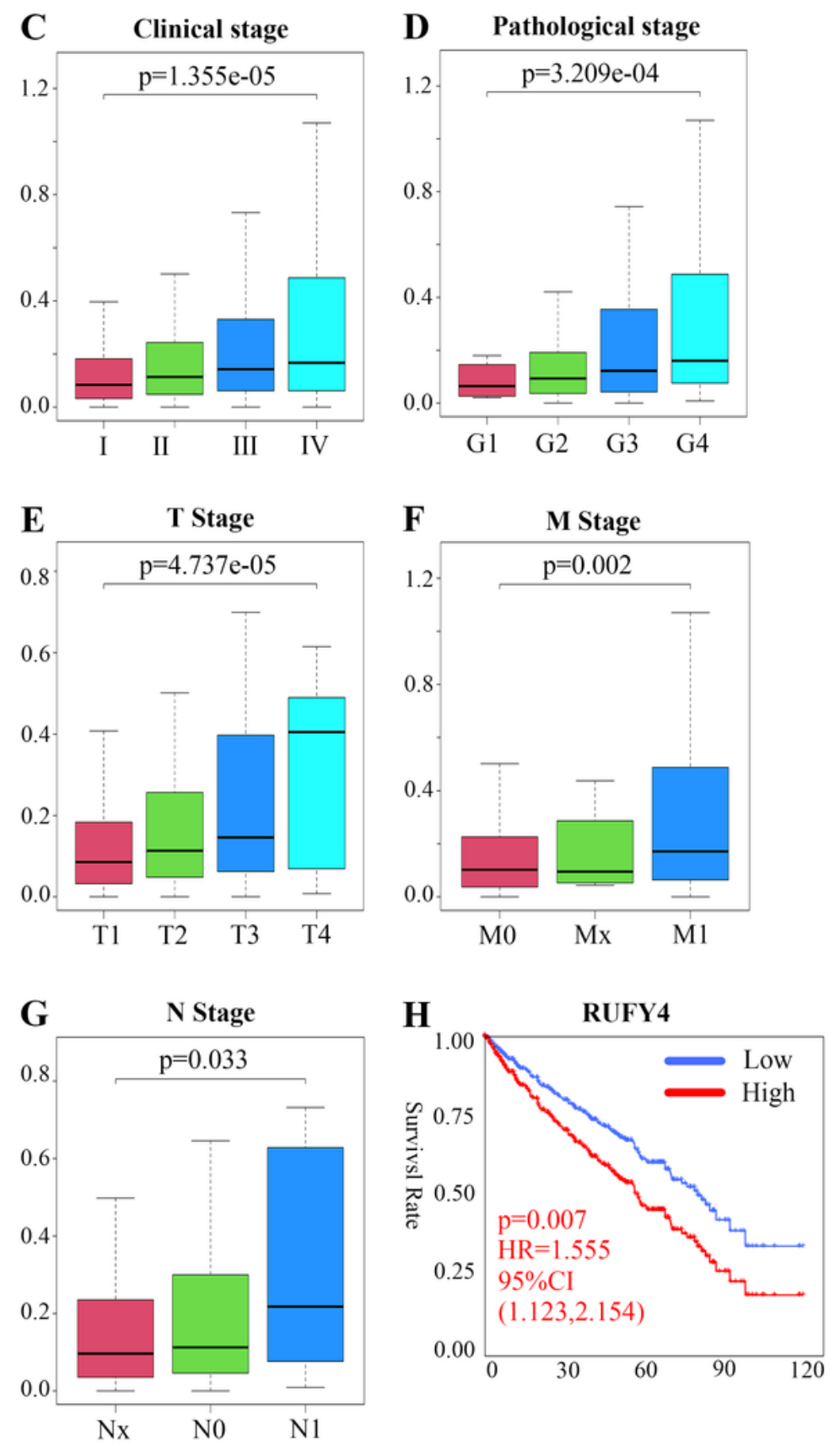

\section{Figure 9}

Validation of the immune correlation and prognostic valve A-B The correlation between RUFY4 and 22 immune cells. The color of each point represented the degree of statistical significance while the size and position of each point represent the correlation coefficient of person and spearman respectively. C-G The relationship between the expression level of RUFY4 and clinical stage, pathological stage, T stage, M 
stage and $\mathrm{N}$ stage. The ordinate represents the content of immune cells and the horizontal line inside the box represents the median value of immune cell contents. $\mathrm{H}$ The multivariate survival analysis for RUFY 4 after adjusting known risk factors.
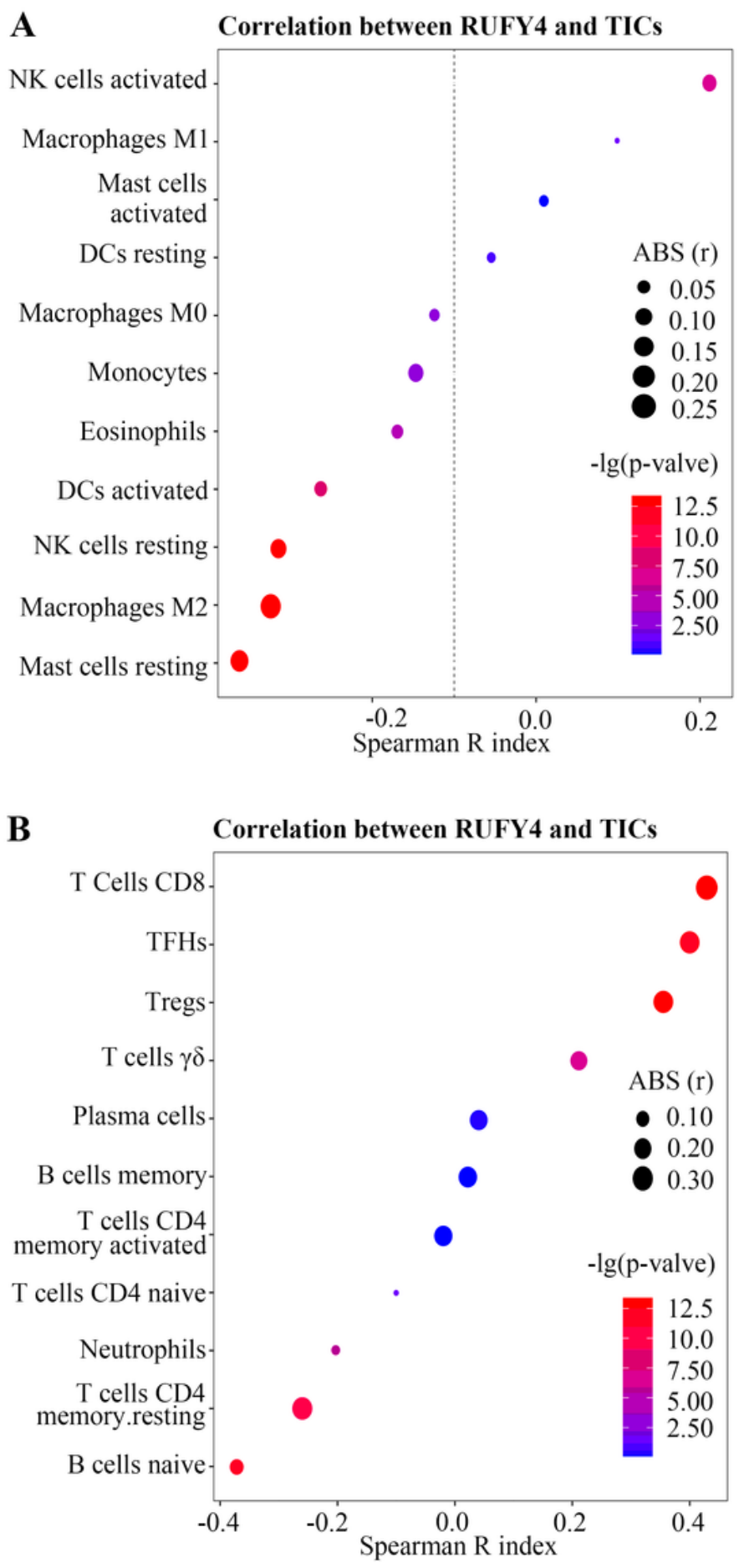
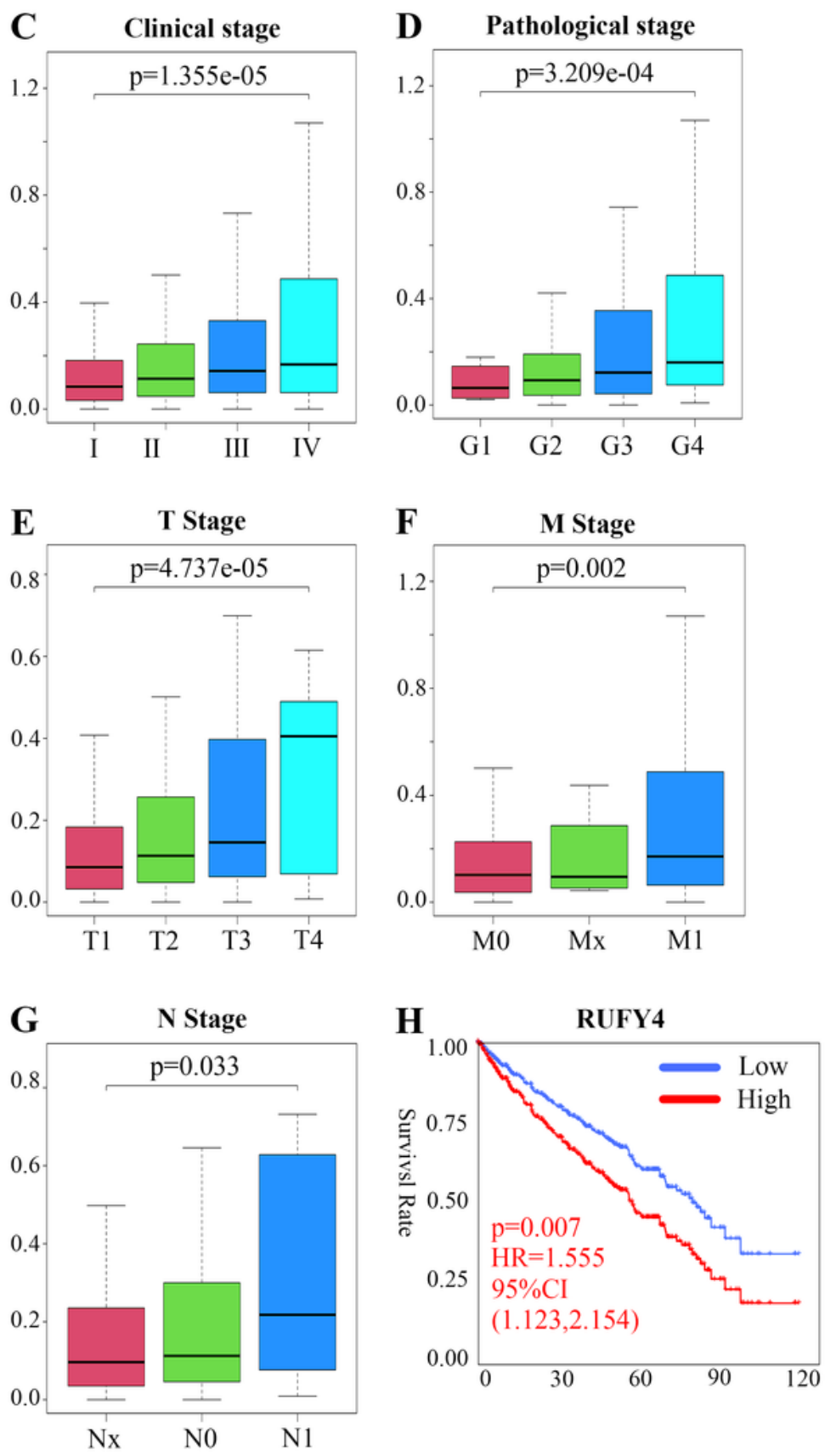

\section{Figure 9}

Validation of the immune correlation and prognostic valve A-B The correlation between RUFY4 and 22 immune cells. The color of each point represented the degree of statistical significance while the size and position of each point represent the correlation coefficient of person and spearman respectively. C-G The 
relationship between the expression level of RUFY4 and clinical stage, pathological stage, T stage, M stage and $\mathrm{N}$ stage. The ordinate represents the content of immune cells and the horizontal line inside the box represents the median value of immune cell contents. $\mathrm{H}$ The multivariate survival analysis for RUFY 4 after adjusting known risk factors.
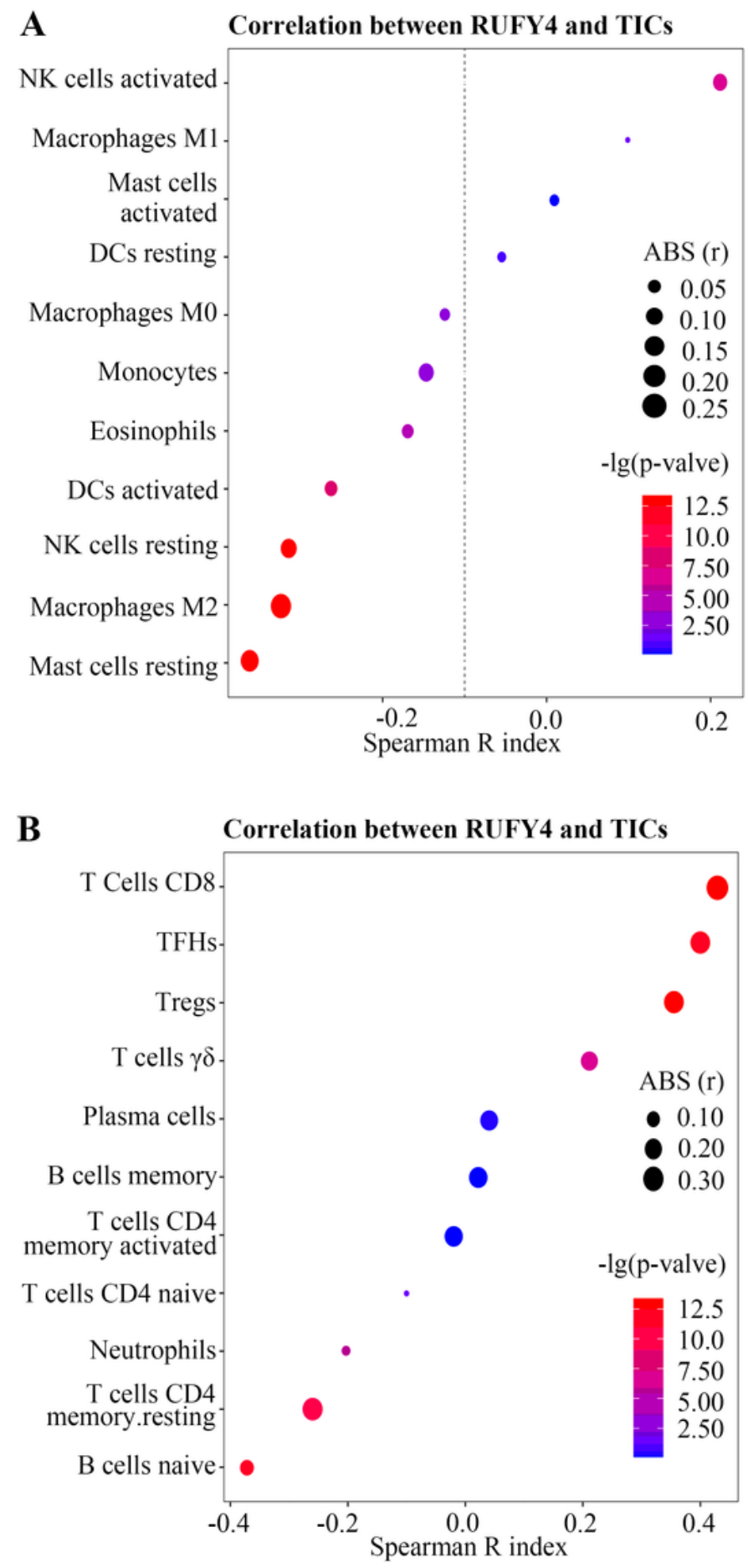
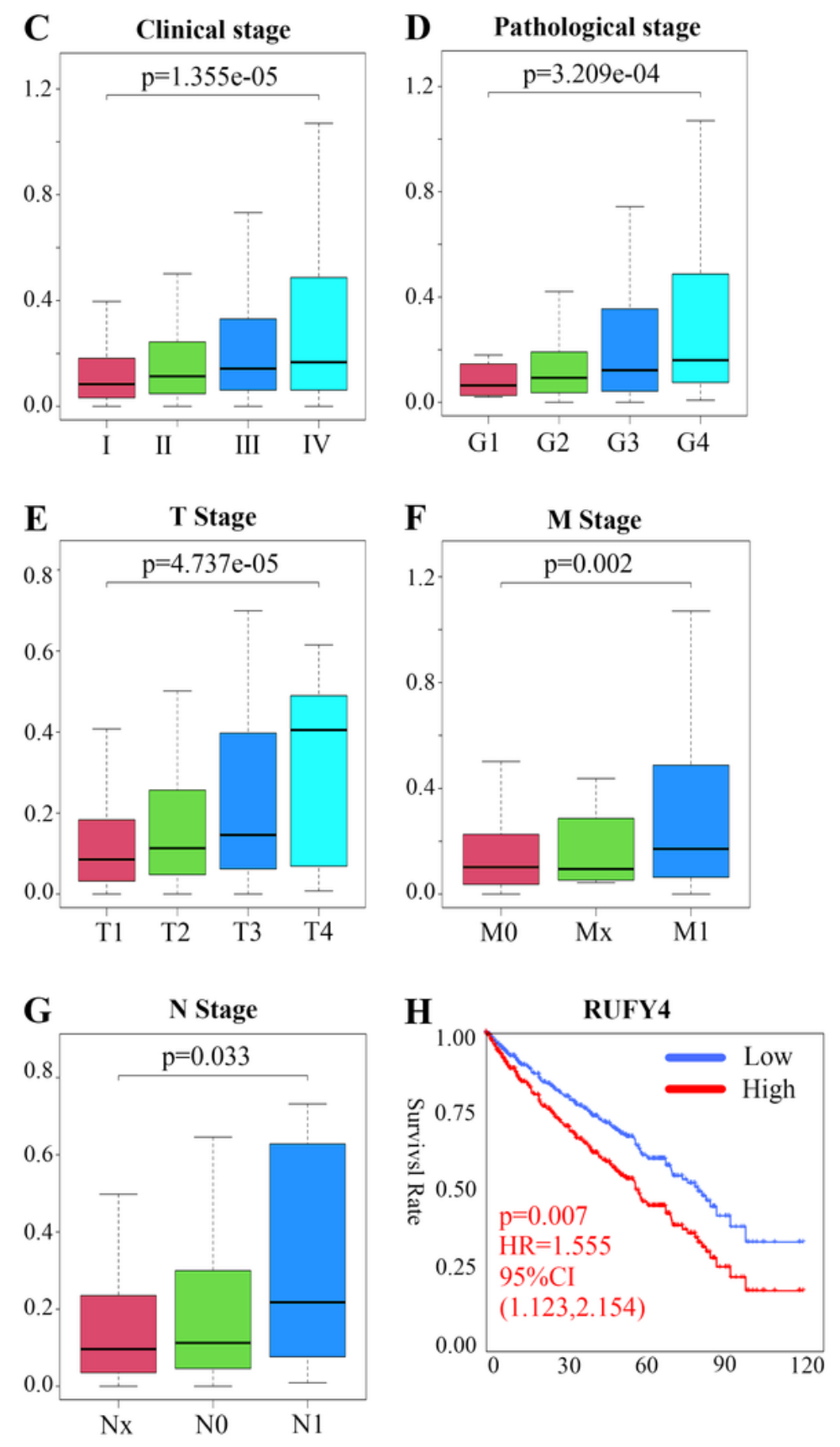

\section{Figure 9}

Validation of the immune correlation and prognostic valve A-B The correlation between RUFY4 and 22 immune cells. The color of each point represented the degree of statistical significance while the size and 
position of each point represent the correlation coefficient of person and spearman respectively. C-G The relationship between the expression level of RUFY4 and clinical stage, pathological stage, T stage, $\mathrm{M}$ stage and $\mathrm{N}$ stage. The ordinate represents the content of immune cells and the horizontal line inside the box represents the median value of immune cell contents. $\mathrm{H}$ The multivariate survival analysis for RUFY 4 after adjusting known risk factors.

\section{Supplementary Files}

This is a list of supplementary files associated with this preprint. Click to download.

- suplementalfigure1.tif

- suplementalfigure1.tif

- suplementalfigure1.tif

- suplementalfigure1.tif

- Table1.xlsx

- Table1.xlsx

- Table1.xlsx

- Table1.xlsx 